University of Louisville

ThinkIR: The University of Louisville's Institutional Repository

Electronic Theses and Dissertations

$12-2014$

\title{
Thermionic emission properties of novel carbon nanostructures.
}

Andriy Sherehiy

University of Louisville

Follow this and additional works at: https://ir.library.louisville.edu/etd

Part of the Physics Commons

\section{Recommended Citation}

Sherehiy, Andriy, "Thermionic emission properties of novel carbon nanostructures." (2014). Electronic Theses and Dissertations. Paper 1767.

https://doi.org/10.18297/etd/1767

This Doctoral Dissertation is brought to you for free and open access by ThinkIR: The University of Louisville's Institutional Repository. It has been accepted for inclusion in Electronic Theses and Dissertations by an authorized administrator of ThinkIR: The University of Louisville's Institutional Repository. This title appears here courtesy of the author, who has retained all other copyrights. For more information, please contact thinkir@louisville.edu. 


\title{
THERMIONIC EMISSION PROPERTIES OF NOVEL CARBON NANOSTRUCTURES
}

\author{
By \\ Andriy Sherehiy \\ BSc. Department of Physics, Rzeszow University, Poland \\ MSc. Department of Physics, Rzeszow University, Poland \\ MSc. Department of Physics and Astronomy, University of Louisville
}

\author{
A Dissertation \\ Submitted to the Faculty of the \\ College of Art and Science of the University of Louisville \\ In Partial Fulfillment of the Requirements \\ For the Degree of
}

Doctor of Philosophy

Department of Physics and Astronomy

University of Louisville

Louisville, Kentucky

December 2014 
Copyright 2014 by Andriy Sherehiy All rights reserved 



\title{
THERMIONIC EMISSION PROPERTIES OF NOVEL CARBON NANOSTRUCTURES
}

\author{
By \\ Andriy Sherehiy \\ BSc. Department of Physics, Rzeszow University \\ MSc. Department of Physics, Rzeszow University \\ MSc. Department of Physics and Astronomy, University of Louisville \\ A Dissertation Approved \\ on 25th November 2014 \\ by the Following Dissertation Committee: \\ Dr. Gamini U. Sumanasekera (Principal Advisor) \\ Dr. Robert W. Cohn (Co-advisor) \\ Dr. Shi $\mathrm{Yu} \mathrm{Wu}$ \\ Dr. C. S. Jayanthi
}




\section{ACKNOWLEDGEMENTS}

I would like to thank my thesis advisor Dr. Gamini Sumanasekera for his support and contributions towards my research. It would not have been possible without his guidance and encouragement. Thank you for patiently sharing all the knowledge. I would like to pay special thanks to Dr. Robert W. Cohn for allowing me to use advanced equipment in his laboratory. I am very grateful to Dr. Mahendra K. Sunkara for his valuable advises, help and most importantly providing access to the CVD laboratory and facilities at the Conn Center for Renewable Energy Research. I wish to thank Dr. Shi Yu Wu for being a great teacher and Dr. C. S. Jayanthi for her support as the chairperson of the Department of Physics and Astronomy.

I would like to thank the entire faculty and staff of the Department of Physics and Astronomy and the Conn Center for Renewable Energy.

I would also like to thank my lab colleagues for their company and support. Especially, I would like to thank Rupa Dumpala, Ruwantha Jayasingha, Jeong Hoon Kim, Chandrashekhar Pendyala, Robert Stallard, Daniel, Alejandro, Ivan, David Mudd, Kasun Fernando, and Dr. Martin Williams. Finally I would like to thank my parents and sister for their constant encouragement and support. 


\section{ABSTRACT \\ THERMIONIC EMISSION PROPERTIES OF NOVEL CARBON NANOSTRUCTURES}

Andriy Sherehiy

November 25, 2014

Materials with low work function values $(<2 \mathrm{eV})$ are highly in demand for low temperature thermionic electron emission, which is a key phenomenon for waste heat recovery applications. Here we present the study of the thermionic emission of the hybrid structure phosphorus, (P) doped diamond nano crystals grown on conical carbon nanotubes (CCNTs). The CCNTs provide the conducting backbone for the P-doped diamond nanocrystals.

In the first part of this thesis thermionic emission properties of conical carbon nanotubes (CCNTs) grown on platinum wires and planar graphite foils were investigated. The work function $(\Phi)$ values extracted from the thermionic emission data range from 4.1 to $4.7 \mathrm{eV}$. The range of $\Phi$ values is attributed to the morphological characteristics, such as tip radius, aspect ratio, density, and wall structure of CCNTs. The observed lower values for $\Phi$ are significantly smaller than that of multi-walled carbon nanotubes (MWNTs). 
The reduced $\Phi$ values are attributed to field penetration effect as a result of the local field enhancement from these structures having high aspect ratio and an excellent field enhancement factor. The high amplification of the external field at the apex of the nanostructures is capable of reducing both the barrier height and the width, in turn contributing to the improved emission current at lower temperatures. The ultraviolet photoemission spectroscopy data of CCNTs grown on Pt wires are in reasonable agreement with the thermionic emission data.

In the next part of the thesis we present work function reduction of phosphorus (P) doped (i) diamond nanocrystals grown on conical carbon nanotubes (CCNTs) and (ii) diamond films grown on silicon substrates. Thermionic emission measurements from phosphorus doped diamond crystals on CCNTs resulted in work function value of 2.23 $\mathrm{eV}$. The reduced work-function is interpreted as due to the presence of the surface states and midband-gap states and no evidence for negative electron affinity was seen. However, Ultraviolet photo-spectroscopy studies on phosphorus doped diamond films yielded a work function value of $\sim 1.8 \mathrm{eV}$ with a negative electron affinity (NEA) value of $1.2 \mathrm{eV}$. Detailed band diagrams are presented to support the observed values for both cases.

In addition we determined the work function values of nanocrystalline $\mathrm{P}$ doped diamond films grown on $\mathrm{W}$ foil to be significantly lower, 1.0- $1.33 \mathrm{eV}$ compared to the hybrid structure and polycrystalline film on Si substrates.

We studied tungsten (W) nanowires as an alternative material in place of CCNT as the supporting and conducting channel for $\mathrm{P}$ doped diamond crystals in a new hybrid structure. 
We described the process of fabrication of arrays of vertical $\mathrm{W}$ nanowires by microwave plasma treatment and synthesis of $\mathrm{P}$ doped nanocrystalline diamond on top of the reduced $\mathrm{W}$ nanowires. Thermionic emission measurements from the alternative hybrid structure resulted in high value of the work function $\sim 5.1 \mathrm{eV}$. 


\section{TABLE OF CONTENTS}

PAGE

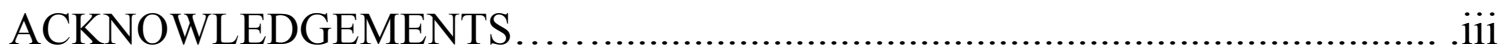

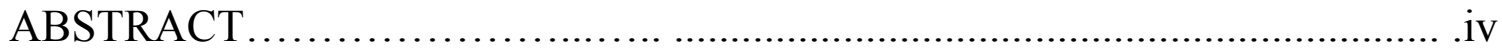

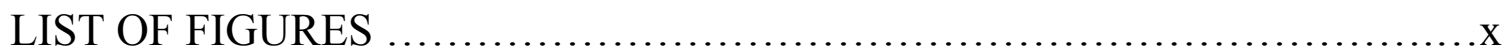

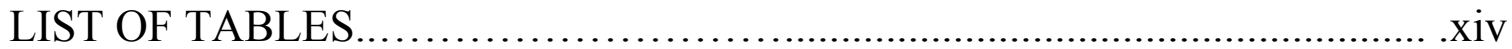

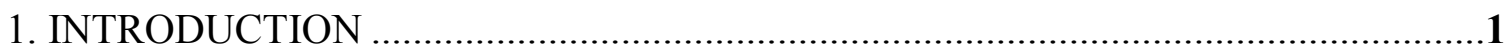

2. THEORETICAL BACKGROUND. FIELD AND THERMIONIC EMSISSION .........4

2.1 Free electron theory of metals. Scattering by 1D barrier ..........................................

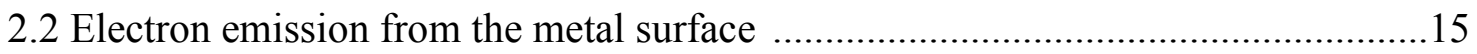

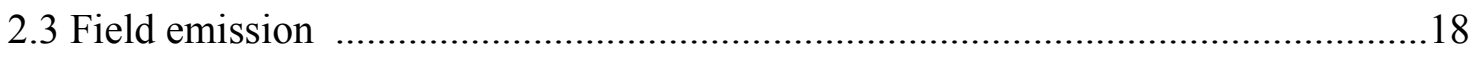

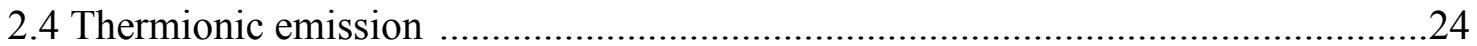

3. WORKFUNCTION OF THE CARBON NANOTUBES

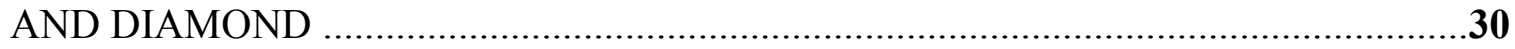

3.1 Work function of the carbon nanotubes and

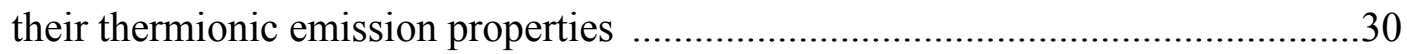

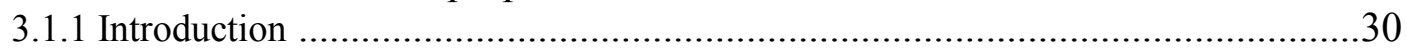

3.1.2 Work function of the CNT - methods of measurement …….................................32

3.1.3 Thermionic emission of the CNT and work function determination .......................37

3.2 Work function of diamond and thermionic emission properties of the diamond films ............................................43

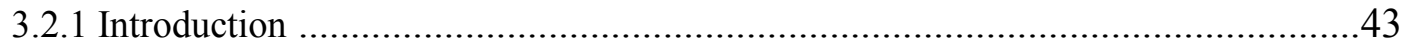

3.2.2 Negative Electron Affinity, $\mathrm{n}$ type doping and thermionic emission ......................45

3.2.3 Electronic properties of the surface of the diamond. Intrinsic and defect states at the

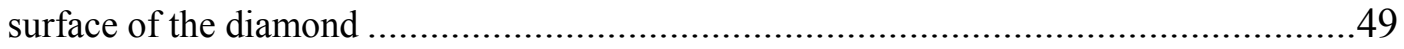

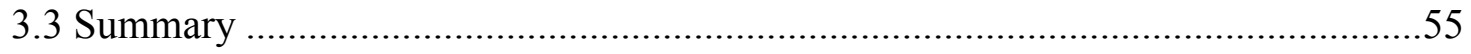




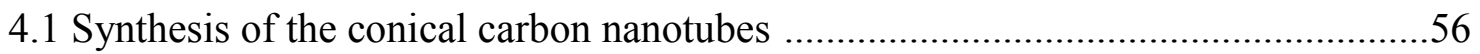

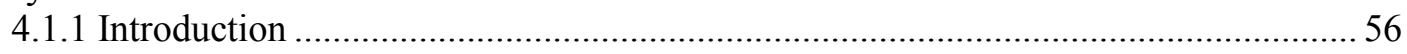

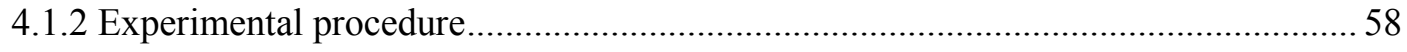

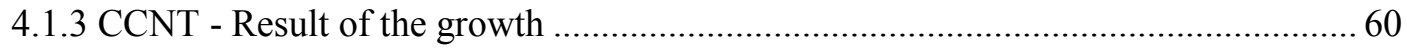

4.2 CCNT - thermionic emission and UPS measurements ......................................62

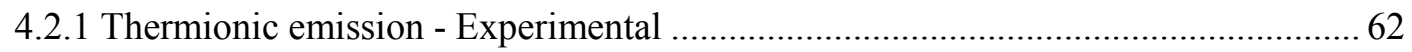

4.2.2 UPS - Experimental ........................................................................................ 64

4.2.3 Thermionic Emission and UPS of the CCNT - Results and discussion ..................... 64

4.2.4 Control measurements and conductivity of the CCNT........................................... 78

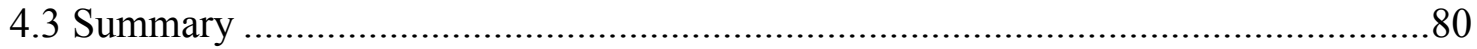

\section{THERMIONIC EMISSION FROM PHOSPHOROUS DOPED} DIAMOND CRYSTALS SUPPORTED BY CONICAL CARBON NANOTUBES. UPS MEASUREMENTS OF THE

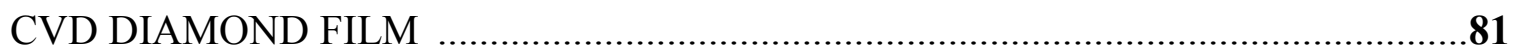

5.1 Synthesis of the P doped diamond crystals and diamond film .................................81

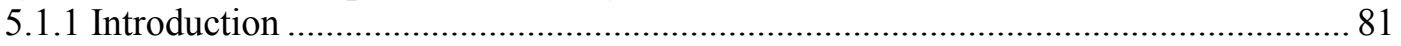

5.1.2 Experimental procedure. Synthesis of the diamond crystals supported by CCNTs and diamond films on Si substrates ..................................... 82

5.1.3 Result of the growth. CCNTs coated with diamond crystals and diamond film on Si substrate .............................................................................. 84

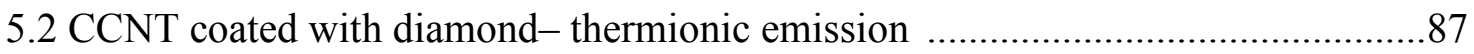

5.2.1 Thermionic emission - Experimental ………………………………………...... 87

5.2.2 Thermionic emission and work function determination -

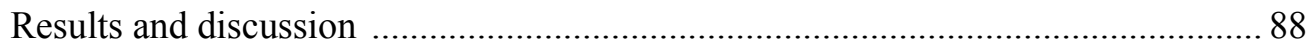

5.3 Ultraviolet Photoelectron Spectroscopy of diamond films grown on

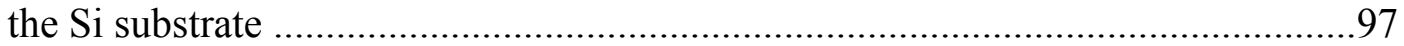

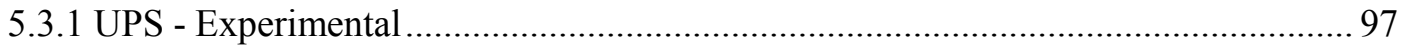

5.3.2 UPS and work function determination of the diamond films -

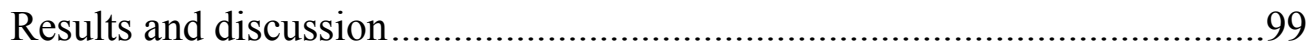

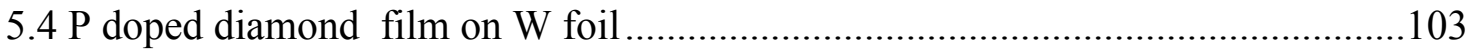

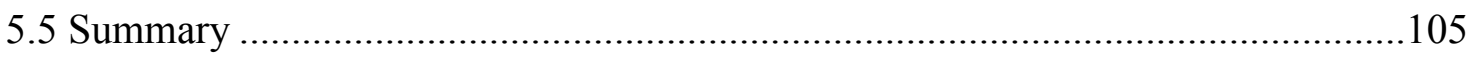




\section{TUNGSTEN OXIDE NANOWIRES. THERMIONIC EMISSION}

PROPERTIES AND WORK FUNCTION

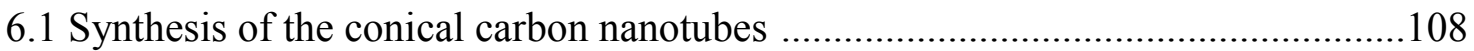

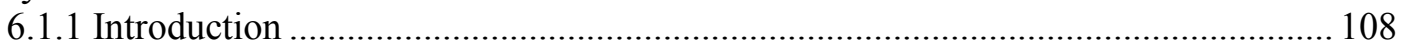

6.1.2 Experimental procedure - synthesis and plasma reduction ..................................... 108

6.1.3 Results $-\mathrm{W}_{18} \mathrm{O}_{49}$ nanowires before and after reduction ....................................... 110

6.1.4 Results $-\mathrm{W}_{18} \mathrm{O}_{49}$ nanowires before and after reduction. XRD, RAMAN, TEM ...112

6.2 Synthesis of the P doped diamond on the W/WOx nanowires

6.3 Thermionic emission measurements of the W/WOx nanowires

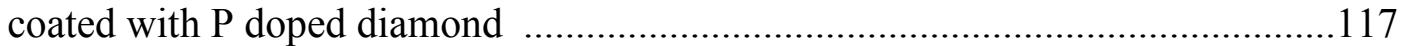

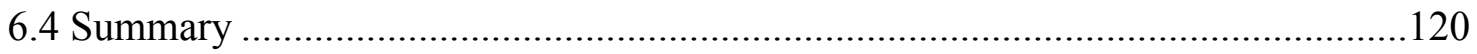

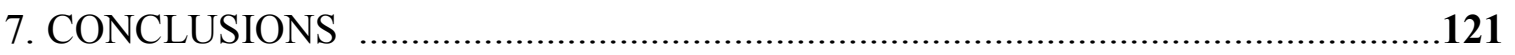

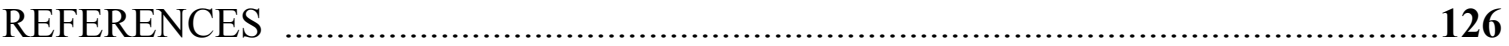

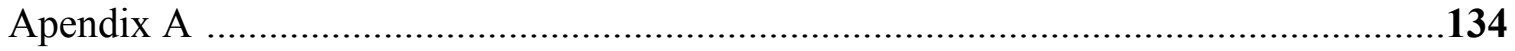

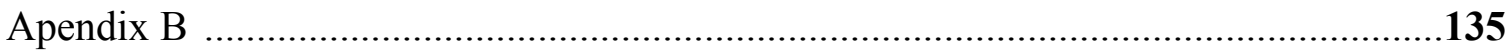

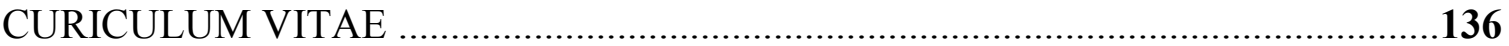




\section{LIST OF FIGURES}

FIGURE

PAGE

1.1 Schematic view of the thermionic emission converter ................................................

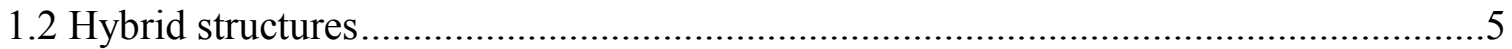

2.1 Schematic view of the considered unit area at metal surface .....................................10

2.2 One dimensional potential barrier........................................................................11

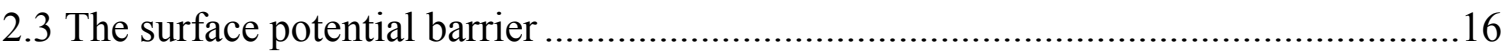

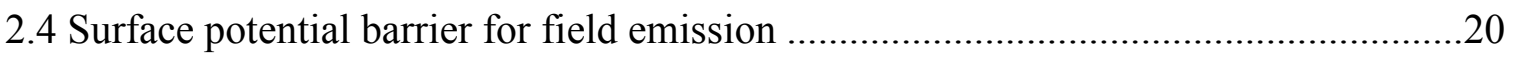

2.5 The electric field lines between two electrodes ....................................................22

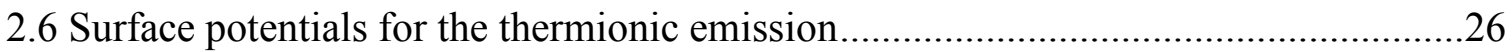

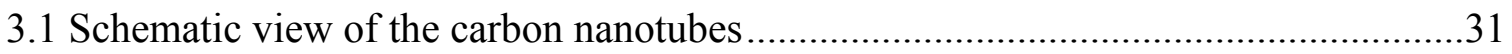

3.2 Crystallographic unit cell (unit cube) of the diamond structure ...................................41

3.3 Lowering of electron affinity of diamond by electrostatic effect of

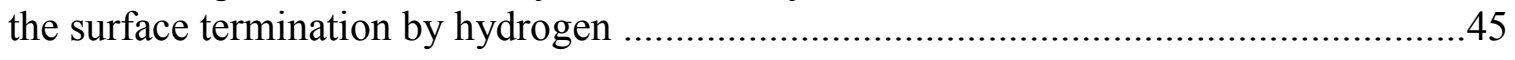

3.4 Donor levels of the different impurities for the diamond ..........................................47

3.5 Illustration of defect induced surface band bending on a p-type diamond according to J.Ristein et al., [42]. A graphite like DOS of the surface is assumed ...........52

4.1 SEM images of the CCNT with the different morphologies including SEM and TEM images of their tips. SEM image of broken individual CCNT ..................................57

4.2 Schematic view of the vacuum chamber for MWCVD growth ...................................59

4.3 SEM images of array of CCNTs on Pt wire and graphite foil ...................................61

4.4 Field emission and thermionic emission measurement set-up....................................62

4.5 Thermionic emission $I-U$ curves collected for CCNT grown Pt ................................65 
4.6 $\operatorname{Ln}(I)$ vs $U^{1 / 2}$ curves and Richardson-Dushman linear fit curve for CCNT grown on $\mathrm{Pt}$

4.7 I - U characteristics, $\operatorname{Ln}(I)$ vs $U^{1 / 2}$ curve and Richardson-Dushman linear fit for CCNT grown on graphite foil

4.8 Richardson-Dushman plots for all three samples grown on the graphite foil (samples 4-6).

4.9 Low kinetic-energy $\mathrm{He}-\mathrm{I}(21.23 \mathrm{eV})$ spectra of CCNT arrays on

platinum wire

4.10 Field emission measurements of the sample 4.

4.11 The field enhancement factor, $\boldsymbol{\beta}$ vs. cathode-anode separation $d$ for the four CCNT representative samples grown on graphite foils

$4.12 I-U$ characteristic at different temperatures for bare graphite foil and

CCNTs on graphite

$4.13 I-U$ characteristic of the array of CCNT grown on the graphite foil in anode-CCNT contact mode

5.1 Experimental set up for growth of diamond crystals

on CCNT arrays

5.2 Scanning electron microscope (SEM) images of individual P-doped diamond coated CCNT grown on graphite foil

5.3 Secondary ion mass spectroscopy (SIMS) depth profile of phosphorus

in P-doped diamond crystals on conical carbon nanotubes

5.4 SEM images of the P doped diamond film grown on the Si substrate

5.5 Secondary ion mass spectroscopy (SIMS) depth profile of phosphorus in P-doped diamond films on silicon wafer

5.6 P doped diamond crystals on CCNTs: (a) Thermionic I-V characteristics

for different temperatures, (b) $\operatorname{Ln}(\mathrm{I}) \mathrm{vs} \mathrm{U}^{1 / 2}$ (c) Richardson_Dushman curve

(d) linear fit of the experimental points

5.7 Thermionic $\mathrm{I}-\mathrm{V}$ characteristics and $\mathrm{Ln}(\mathrm{I}) \mathrm{vs} \mathrm{U}^{1 / 2}$ plots for bare cent and undoped diamond

5.8 Thermionic emission data (dots) and data-fit (line) to Richardso-Dushman equation from thermionic emitters based on bare CCNTs (red), CCNT coated with undoped diamond (green) and CCNT coated with phosphorus doped diamond ( blue) 
5.9 Surface energy band diagram of P doped and undoped diamond grown on CCNTs ..94 5.10 Schematic view of band diagram illustrating the band bending

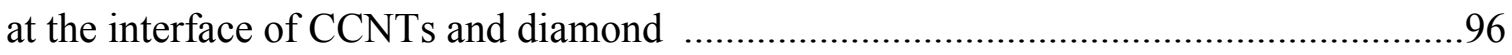

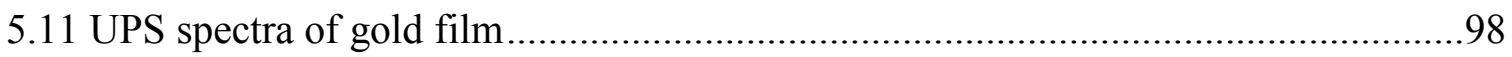

5.12 UPS spectra of low kinetic-energy part of $\mathrm{He}-\mathrm{I}(21.23 \mathrm{eV})$ spectra undoped diamond film and phosphorus doped diamond film

5.13 Surface energy band diagrams for $\mathrm{P}$ doped and undoped diamond films grown on $\mathrm{Si} / \mathrm{DiO}_{2}$

5.14 SEM images of the P doped nanocrystalline film grown on the $\mathrm{W}$ substrate

5.15 Thermionic emission measurements from $\mathrm{P}$ doped film grown on $\mathrm{W}$ foil 105

6.1 Schematic of the Scale up Hot Filament CVD reactor 109

6.2 SEM images pf Tungsten Oxide nanowires grown on the $\mathrm{W}$ foils

6.3 Tungsten oxide nanowires before and after hydrogen plasma treatment

6.4 XRD spectra of the $\mathrm{W}_{18} \mathrm{O}_{49}$ nanowires before and after hydrogen plasma treatment

6.5 Raman spectra of the $\mathrm{W}_{18} \mathrm{O}_{49}$ nanowires before and after hydrogen

plasma treatment

6.6 SAED, TEM and EDAX analysis of the Tungsten Oxide nanowires

6.7 SEM images of the $\mathrm{P}$ doped diamond nanocrystallien aglomerations

grown on the reduced WOx nanowires.

6.8 Thermionic emission current versus applied voltage at different temperatures for W/WOx nanowire coated with P doped. b) Richardson-Dushman linear fit 118

6.9 Surface energy band diagram of $\mathrm{P}$ doped nanocrystals grown on the $\mathrm{W} / \mathrm{WO}_{\mathrm{x}}$ nanowires. 


\section{LIST OF TABLES}

TABLE

PAGE

1.1 Best estimate of the measured values of $\phi$ for some faces of Tungsten

3.1 Theoretical work function values of carbon nanotubes

and carbon materials

3.2 Values of work function of carbon nanotubes and other carbon

materials obtained by different experimental methods

3.3 Values of work function of carbon nanotubes and other carbon

materials obtained by different experimental methods .....................................................38

3.4 Experimental values of the electron affinity for different diamond faces ....................46

3.5 Values of work function of doped diamond films ...................................................49

4.1 Characteristics of the CCNTs grown on Pt wire and graphite foil ..............................61

4.2 Summary of the work function values of each CCNT sample grown

on graphite foil and on platinum substrate.

4.3 Estimated values of current densities for the whole sample and for individual CCNT 


\section{CHAPTER 1}

\section{INTRODUCTION}

Progress in micro and nanotechnology followed by the development of new classes of materials in the recent $10-20$ years provided new possibilities in a number of disciplines. The advancement in synthesis and characterization techniques allowed better control of structural properties of the synthesized materials at micro and nanoscale. Some examples of such materials are carbon nanotubes $(\mathrm{CNT}) /$ conical carbon nanotubes (CCNT), synthetic diamond grown by chemical vapor deposition (CVD), and nanowires (both semiconducting and metallic). On the other hand, advancement in measurement techniques allowed the study of the electronic transport or electron emission in the mentioned materials. It is expected that on the micro and nanoscale these structures would behave differently than their macroscopic bulk counterparts. This fact, combined with knowledge about specific structural properties of the novel structures, have revitalized the interest of the phenomena well known for decades and thermionic emission (TE) from such structures is the primary focus of this work.

Thermionic emission is the release of charge carriers from the surface of the material upon increasing of the temperature of the material. Thermal energy supplied to the carriers allows them to overcome the surface potential barrier which is equal to the 
work function of a given material. In this work the charge carriers are electrons, however in some cases which is outside of the scope of this study, it could be ions.

Thermionic emission first was observed by F.Guthrie in 1873 [1] and towards the end of $19^{\text {th }}$ centuries by a few other researchers -- including Edison -- who utilized this effect in his first constructed vacuum lamp. In 1901 Richardson introduced his empirically deduced equation for the emission current density from the metal surface [1]

$$
J(T)=A_{0} T^{2} e^{-\frac{\phi}{k_{B} T}}
$$

where, $A_{0}$ is Richardson constant, $T$ is the temperature in $\mathrm{K}, k_{B}$ is the Boltzman constant and $\phi$ is the work function. Nordheim and Sommerfield later derived the similar emission formula using Fermi-Dirac distribution and newly developed quantum mechanical formalism [1]. Even though this formula has existed for over 100 years it is still being used to describe thermionic emission.

Thermionic emission has had numerous applications, usually as a source of the electrons in vacuum electronics, vacuum lamps, ionization pressure gauges, X-ray tubes, filaments in Electron Microscopes, as well in magnetrons (source of microwaves). Conversion of heat to useful energy(direct conversion of the heat energy into the electrical energy by utilizing thermionic emission [2]) is another important application of thermionic emission.

Figure 1.1 represents a schematic view of the thermionic emission converter (TEC), where emitter temperature is elevated (in contact with heat source) emits electrons to vacuum gap which are collected by the collector located at some separation 
distance from the emitter and kept at much lower temperature. By attaching the load to the electrodes in the circuit, an electric current will be generated assuming there is a constant supply of heat energy to the emitter. The work function of the emitter has to be larger than that of the collector. The optimal value of the work function for the emitter should be $\leq 2 \mathrm{eV}$ while that for collector should be as low as $\sim 1 \mathrm{eV}$ to be used as a thermionic converter at low enough temperatures [2].

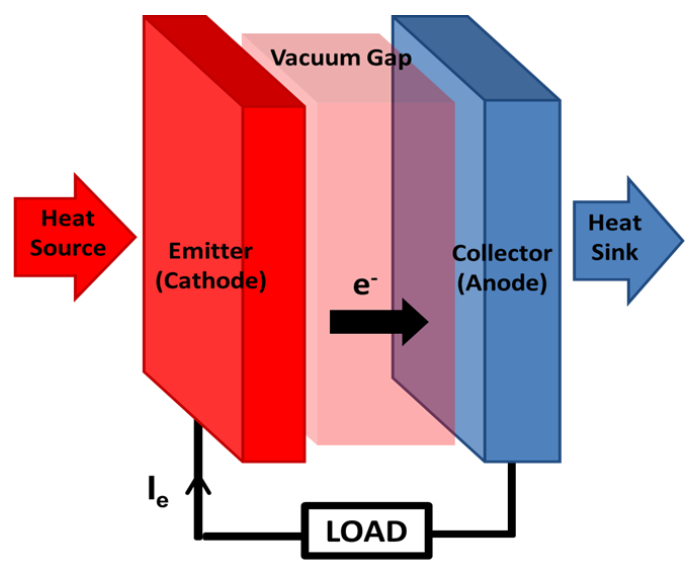

Figure 1.1 Schematic view of the thermionic emission converter

It is evident from Richardson eq (1.1) that efficient emission to the vacuum depends on the temperature and the work function. Thus the efficiency of the TEC depends on the work functions of the material used for the collector and emittor

Another important condition required thermionic emitter materials is its stability at high temperatures $\sim 1000 \mathrm{~K}$ or lower depending on the work function. Thus the suitable material for the hot cathode in thermionic emission needs to satisfy these two conditions: low work function $(\leq 2 \mathrm{eV})$ and the stability in the high temperature range $700-1000 \mathrm{~K}$. 
Currently available materials which satisfy the above conditions include Thoriated Tungsten, porous Tungsten impregnated with Ba based multi component oxide, coated cathodes $(\mathrm{BaO}, \mathrm{SaO}, \mathrm{CaO})$, boride cathodes $\left(\mathrm{LaB}_{6}\right.$ and the whole family of the hexaboride materials). An alternative candidate for cathode material is Nitrogen $(\mathrm{N})$, Sulfur (S) or Phosphorous (P) doped diamond with Negative Electron Affinity (NEA) [3]. Diamond has unique features which include high thermal conductivity, high breakdown electric field, high mechanical hardness and low coefficient of thermal expansion, and resistance to harsh environments including radiation, chemicals, and corrosion [4]. One thermionic emission study has shown that $\mathrm{P}$ doping of a diamond film together with the presence of NEA exhibit lower work function of $\sim 0.9 \mathrm{eV}$ [5]. It is the lowest reported work function value for doped diamond and remains one of the lowest among all other known materials.

In search for the structure suitable for the thermionic emission conversion, a different approach utilizing properties of the micro/nanostructures was attempted. Specifically, a hybrid structure consisting of the conical carbon nanotubes (CCNT) coated with P doped diamond crystals Figure $2 \mathrm{a}[6,7]$ is proposed in this work. It was suggested that such hybrid architecture has an essential and unique property - large surface area(high surface to volume ratio) - which could improve the emission properties of doped diamond crystals and improve the incorporation of dopants in to the diamond crystals. An alternative hybrid structure consisting of arrays of W nanowires coated with P doped diamond crystals Figure 1.2b is also proposed. 
a)

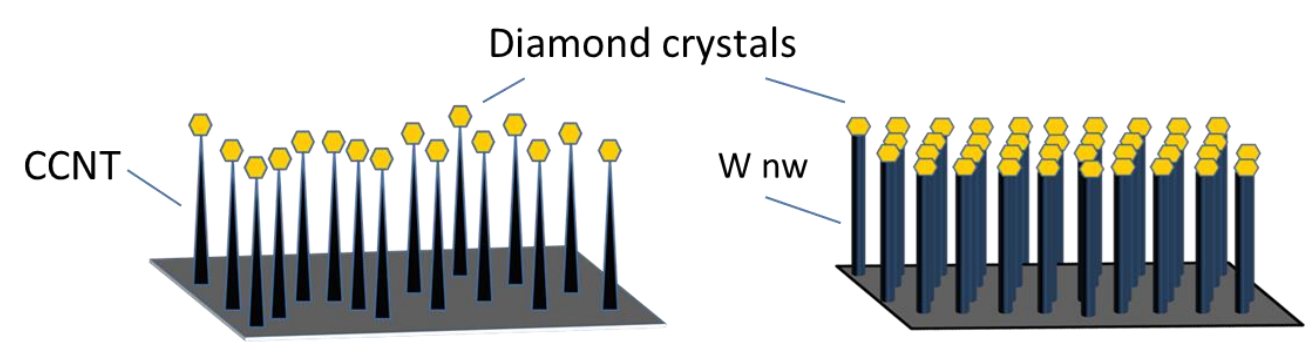

Figure 1.2 Hybrid structure of the: a) CCNT coated with diamond crystals; b) W nw coated with diamond crystals.

The goal of this work is to study the thermionic emission from the array of CCNT grown on different substrates (Pt wire and graphite foil) and determination of the work function of the structures. The study also includes the examination of the dependence of the work function on the morphology of the CCNT arrays based on their characterization and analysis of the field emission (FE) measurements. Next, the study is concerned with thermionic emission from the CCNT coated with $\mathrm{P}$ doped diamond crystals and determination of the work function of the diamond crystals. The study also includes the understanding of the mechanism behind the origin of such work function values. Ultraviolet photoelectron spectroscopy of the P doped diamond films (instead of diamond crystals) is meant to be complementary to the thermionic emission measurements of the hybrid structure.

The next phase of the study involves thermionic emission measurements of the hybrid structure based on tungsten (W) nanowires decorated with $\mathrm{P}$ doped diamond crystals. This part involves synthesis of the WOx nanowire array, reduction to the metallic $\mathrm{W}$ nanowires and growth and doping of diamond crystals, and finally thermionic emission measurements on $\mathrm{W}$ nanowires and $\mathrm{W}$ nanowires decorated with $\mathrm{P}$ doped diamond. 
Chapter 2 presents the theoretical background of the thermionic and field emission mechanisms including the free electron model approximation of field and thermionic emission with a discussion on its applicability and limitations.

Chapter 3 reviews work function values of the carbon based materials determined by different methods. Specifically thermionic emission studies of carbon nanotubes and diamond films are presented and analyzed in this chapter.

Chapter 4 is dedicated to conical carbon nanotubes. Structural properties, experimental details about synthesis and thermionic and field emission measurements are discussed. In this chapter, the results of the FE and TE measurements are presented along with their analysis and discussion.

In chapter 5 we concentrate on the hybrid structure of the CCNT coated with P doped diamond, $\mathrm{P}$ doped polycrystalline diamond film on $\mathrm{Si}$ substrate and $\mathrm{P}$ doped nanocrystalline diamond film on $\mathrm{W}$ substrate presenting experimental details about synthesis of these structures, doping and TE measurements case of diamond films, UPS measurements are presented. We discuss the results of the work function values- and suggest underlying mechanism leading to such values of $\phi$.

Chapter 6 is dedicated to second hybrid structure of the W nanowires coated with P doped diamond films with a detailed description of the synthesis and WOx nanowires, reduction process to $\mathrm{W}$ nanowires, and doped diamond growth on the W/WOx nanowire array. Results of the thermionic emission measurements are presented as well. 


\section{CHAPTER 2}

\section{THEORETICAL BACKGROUND: FIELD AND THERMIONIC EMISSION}

In this chapter a review of the theory of the two basic types of electron emission (field and thermionic) is presented. Description of the FE and TE is based on the free electron theory. Field emission first described by Fowler and Nordheim is a direct result of quantum tunneling of electrons through a surface potential barrier in the presence of a strong electric field. Thermionic emission is generally described in two slightly different processes: real thermionic emission at high temperatures $(\sim 1000 \mathrm{~K})$ with no external electric fields and field enhanced thermionic emission (Schottky emission) also at high temperatures in the presence of a weak electric field resulting in a small reduction of the surface image potential.

\subsection{Free electron theory of metals. Scattering by 1D barrier.}

The free electron theory of metals [8] is the basis for the traditional quantummechanical theories of field and thermionic emission from metals [9]. Some important formulas derived on the basis of this theory have been used in the analysis of 
experimental results until recently. We, therefore, would like to provide a brief description of free electron theory of electron emission phenomena.

The free electron theory is based on the assumption that electrons occupying states in conduction band behave as free particles. In this model, electron states are described by plane wave function

$$
\psi_{k}(\boldsymbol{r})=\frac{1}{L^{3 / 2}} e^{i \boldsymbol{k} \boldsymbol{r}}
$$

where, $L^{3} \equiv V$ is the metal volume (assumed to be very large) and $\boldsymbol{k}$ is the wave vector

$$
\boldsymbol{k}=\frac{2 \pi}{L}\left(n_{x}, n_{y}, n_{z}\right)
$$

where, $\mathrm{n}_{\mathrm{x}}, \mathrm{n}_{\mathrm{y}}, \mathrm{n}_{\mathrm{z}}$ are positive or negative integers. Energy of the electron occupying particular state $\psi_{k}(\boldsymbol{r})$ is given by

$$
E_{k}=\frac{\hbar^{2} k^{2}}{2 m}
$$

where, $m$ is the mass of the electron. A zero value of energy is aligned with the bottom of the conduction band. For a complete description, it is important to specify that for a given $\boldsymbol{k}$ there are two states with different spin orientation of the electron (up, down) with the same spatial wave function (1.1). Considering that $L$ extends to infinity, the number of electron states per unit volume with energies between $E$ and $E+d E$ is given by 


$$
\rho(E) d E=\frac{2}{(2 \pi)^{3}} \iiint_{E}^{E+d E} d^{3} k=\frac{1}{2 \pi^{2}}\left(\frac{2 m}{\hbar^{2}}\right)^{3 / 2} E^{1 / 2} d E
$$

The probability that an electron state with energy $E$ is occupied is calculated using FermiDirac distribution function

$$
f(E)=\frac{1}{1+\exp \left[\frac{E-E_{F}}{k_{B} T}\right]}
$$

where $k_{B}$ is the Boltzmann constant, $E_{F}$ is the Fermi level and $T$ is the absolute temperature. Fermi level could be determined from the requirement defined by given integral

$$
\int_{0}^{\infty} \rho(E) f(E) d E=N_{C}
$$

where, $\mathrm{N}_{\mathrm{C}}$ is the number of conduction band electrons per unit volume (concentration).

In describing the electron emission from the metal's surface, one must define a more specific form for the electron concentration. According to Modinos [9] let us consider the case under equilibrium conditions, where the number of electrons which cross a unit area (in $x y$ plane, Fig 1.1), in z direction (from left to right), per unit time, where total energy is in range $[E, E+d E]$, and normal energy is defined by

$$
W=\frac{\hbar^{2} \mathrm{k}_{\mathrm{z}}^{2}}{2 \mathrm{~m}}
$$


Between $W$ and $W+d W$. Number of electrons defined in such manner is denoted by $N(E, W) d E d W$ and is expressed by

$$
\begin{aligned}
N(E, W) d E d W & =\frac{2 f(E)}{(2 \pi)^{3}} \iiint_{\{E, W\}} v_{z} d^{3} k \\
& =\frac{m}{2 \pi^{2} \hbar^{3}} f(E) d E d W
\end{aligned}
$$

where,

$$
v_{z}=\frac{1}{\hbar} \frac{\partial E_{k}}{\partial k_{z}}=\frac{\hbar k_{z}}{m}
$$

is the velocity of the electrons normal to the considered unit area. Limits $\{E, W\}$ in (1.9) indicate that in the integration are included only electronic states with energies between $E+d E$ and $W+d W$ corresponding to $v_{z}>0$.

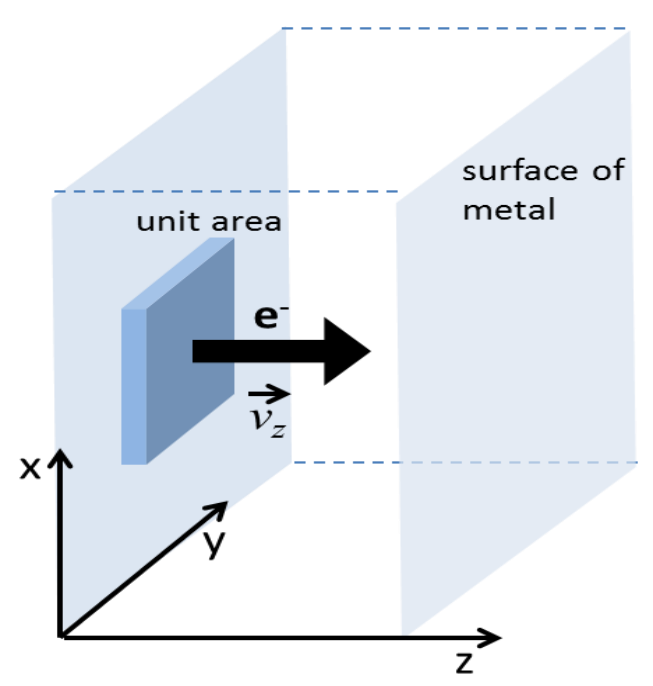

Figure 2.1 Schematic view of the considered unit area at metal surface.

The general qualitative definition of the field and thermionic emission (presented in introduction) implies that for quantum mechanical description of electron emission 
from metal surface it is required first to consider general case of scattering of electron at a potential barrier. Following Modinos [9], let us introduce a potential barrier, $V(z)-$ shown in Figure 1.2 - and let electrons with total energy $\mathrm{E}$ and wave vector $\mathbf{k}_{\|}=\left(\mathrm{k}_{\mathrm{x}}, \mathrm{k}_{\mathrm{y}}\right)$ be incident on this barrier from left.

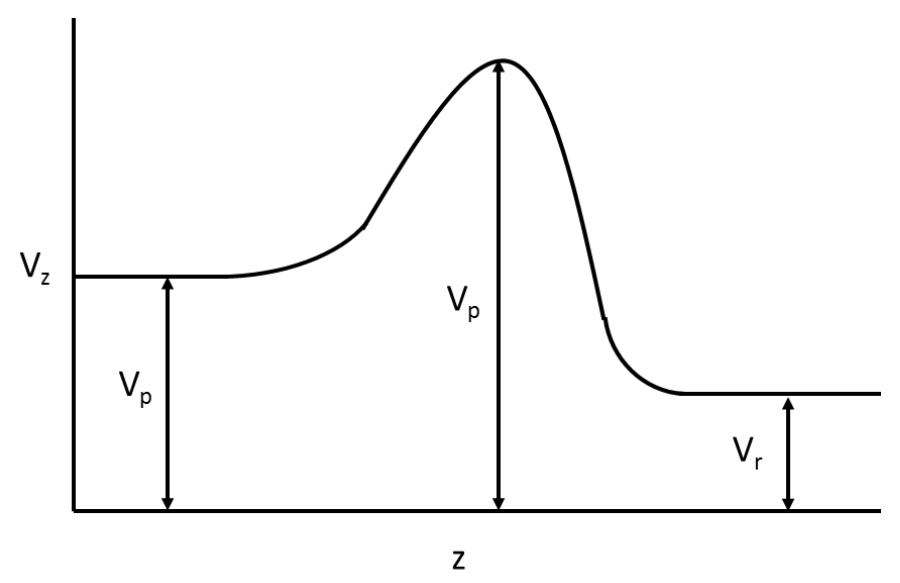

Figure 2.2 One dimensional potential barrier.

Schrodinger equation for this particular case has the following form,

$$
-\frac{\hbar^{2}}{2 m} \frac{d^{2} u}{d z^{2}}+V(z) u=W u(z)
$$

and is satisfied by $u(z)$ of the electronic wave function which has following form (in our case),

$$
\psi=u(z) e^{i \boldsymbol{k}_{\|} \boldsymbol{r}_{\|}}
$$


where, $\boldsymbol{r}_{\|}=(x, y)$. The normal energy here $W=E-\frac{\hbar^{2} k_{\|}^{2}}{2 m}>V_{L}$ and could lie above or below the top of the barrier $V_{p}$. The transmitted wave propagating in the positive $z$ direction is described by the function,

$$
u(z)=C e^{i q_{R} z}
$$

where $V=V_{R}$ is the constant potential to the right of the barrier (Fig 1.2) and

$$
q_{R}=\left[\frac{2 m}{\hbar^{2}}\left(W-V_{R}\right)\right]^{\frac{1}{2}}
$$

In the region of the constant potential $V=V_{L}$ on the left side of the barrier, wave function has the form

$$
u(z)=A e^{i \boldsymbol{q}_{R} z}+B e^{-i \boldsymbol{q}_{R} z}
$$

where

$$
q_{L}=\left[\frac{2 m}{\hbar^{2}}\left(W-V_{L}\right)\right]^{\frac{1}{2}}
$$

In the expression (1.11), the first term represents the incident wave and the second term represents the reflected wave.

In order to determine incident, reflected, transmitted current densities it is needed to use the formula for probability current density vector [10] 


$$
\boldsymbol{j}=\frac{i \hbar}{2 m}\left(\Psi \nabla \Psi^{*}-\Psi^{*} \nabla \Psi\right)
$$

In our case where we consider motion along $z$ direction [9]

$$
j_{z, \alpha}=\frac{\hbar}{2 m i}\left(f_{\alpha}^{*} \frac{\partial}{\partial z} f_{\alpha}-f_{\alpha} \frac{\partial}{\partial z} f_{\alpha}^{*}\right)
$$

where $f_{\alpha}$ denotes the incident, reflected, and transmitted wave.

Using this expression, one can find transmission and reflection coefficients. The transmission coefficient by definition is of such form [9]

$$
D(W) \equiv \frac{j_{z, t r a}}{j_{z, \text { inc }}}=\frac{q_{R}}{q_{L}}\left|\frac{C}{A}\right|^{2}
$$

and the reflection coefficient

$$
R(W) \equiv \frac{j_{z, r e f}}{j_{z, \text { inc }}}=\left|\frac{B}{A}\right|^{2}
$$

Explicit expressions for $\mathrm{D}(\mathrm{W})$ and $\mathrm{R}(\mathrm{W})$ could be obtained analytically only for a few specific potential barriers. However, it should to be noted that $\mathrm{D}(\mathrm{W})$ and $\mathrm{R}(\mathrm{W})$ could be obtained numerically for any barrier which depends only on the $z$ coordinate (Fig 1.2). 
Under the condition that potential barrier decreases slowly and monotonically to the right of the barrier top, the ordinary WKB approximation is valid [10] and the wave function (as transmitted wave) in this region $\left(z \geq z_{r}\right)$ has the form

$$
u(z)=\frac{C}{[\lambda(z)]^{1 / 2}} \exp \left[i \int_{z_{r}}^{z} \lambda(z) d z\right]
$$

where

$$
\lambda(z) \equiv\left[\frac{2 m}{\hbar^{2}}(W-V(z))\right]^{1 / 2}
$$

Modinos notes that for general analysis of the electron emission phenomena it is more preferable to have an analytic expression for the transmission coefficient obtained by less accurate method than numerical one. Miller and Good developed such a method which is a generalization of the ordinary WKB method. This method is valid for certain limiting conditions [9] - when satisfied it provides a good approximation the following formula for transmission coefficient

$$
D(W)=[1-\exp (-A(W))]^{-1}
$$

where

$$
A(W)=2 i \int_{z_{1}}^{z_{2}} \lambda(\xi) d \xi
$$

where, $\lambda(\xi)$ is given by eq. (1.15), $z_{1}$ and $z_{2}$ are the roots of the eq. $W-V(z)$. These general formulas are applicable for the cases when normal energy $\mathrm{W}$ is above or below the top of the barrier and there are no zeros or singularities in the vicinity of $z_{1}, z_{2}$. When 
$W>V(z) z_{1}, z_{2}$ roots are real; for $W<V(z) z_{1}, z_{2}$ become complex roots, the one being conjugation of the other.

\subsection{Electron emission from the metal surface}

Let us consider a semi-infinite surface of the metal. According to the free electron theory of metals, an electron inside the metal stays at constant potential. A minimum energy (at absolute zero) must be supplied to the metal in order to allow an electron to escape from it. minimum energy required is equal to the work function $\varphi$ which is the energy difference between the Fermi level and the top of the surface potential barrier at absolute zero (Fig 1.3). We know from electrostatics that an electron placed at finite distance from the plane surface of a conductor is attracted to it and experiences the "image" potential given by $k \mathrm{e} / 4 z$ (where $k=\frac{1}{4 \pi \varepsilon_{0}}$ and $\varepsilon_{0}=8.854 \cdot 10^{-12} \frac{F}{\mathrm{~m}}$ ). Thus the potential energy of the electron in the vacuum outside of the metal is asymptotically described by the given equation

$$
V(z) \simeq E_{F}+\phi-k \frac{e^{2}}{z}
$$

It is agreed that above equation is valid for $z>3 \AA$, if we consider the metal surface [9]. In the case of the presence of an external electric field $F$ outside of the metal surface we need to add a term to the above eq. - $e F z$

$$
V(z)= \begin{cases}E_{F}+\phi-k \frac{e^{2}}{z}-e F z & \text { for } z>0 \\ 0 & \text { for } z<0\end{cases}
$$


The above equation describes a potential for the electron emission in the presence of an external electric field outside $(z>0)$ and within $(z<0)$ the metal. Fig. 1.3 presents a schematic illustration of the potential $V(z)$.

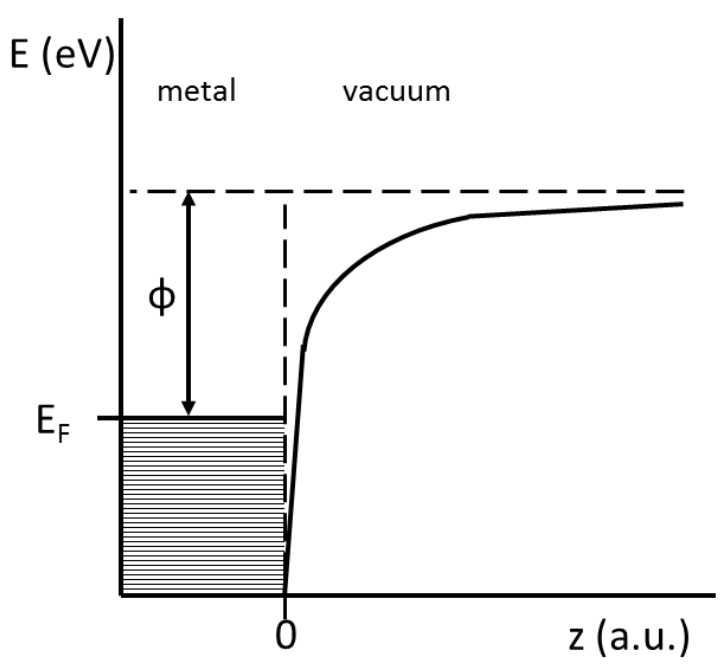

Figure 2.3 The surface potential barrier. Image potential is represented by solid curve.

The number of electrons (within metal) with a normal energy between $W$ and $W+$ $d W$ scattered on the surface barrier is given by

$$
N(W, T) d W=\frac{m k_{B} T}{2 \pi^{2} \hbar^{3}} \ln \left[1+\exp \left(-\frac{W-E_{F}}{k_{B} T}\right)\right] d W
$$

Then the current density will be given by

$$
J(F, T)=e \int_{0}^{\infty} N(W, T) D(W) d W
$$


where $F, T$ denotes the applied electric field and the temperature respectively. $D(W)$ is the transmission probability of electrons scattered at the surface potential barrier. Using eq. (1.16) and (1.17) one can determine the form of the transmission coefficient $D(W)$ for the given potential (1.18a) and in result obtain the general expression for the emission current density [9]

$$
\begin{aligned}
J(F, T) & =e \int_{0}^{\infty} N(W, T) D(W) d W \\
& =\frac{e m k_{B} T}{2 \hbar^{3} \pi^{2}}\left\{\int_{0}^{W_{l}} \frac{\ln \left[1+\exp \left(-\frac{W-E_{F}}{k_{B} T}\right)\right]}{1+\exp [Q(W)]} d W\right. \\
& \left.+\int_{W_{l}}^{\infty} \ln \left[1+\exp \left(-\frac{W-E_{F}}{k_{B} T}\right)\right] d W\right\}
\end{aligned}
$$

where,

$$
\begin{gathered}
Q(W)=-2 i \int_{z_{1}}^{z_{2}} \lambda(\xi) d \xi \\
\lambda(\xi)=\left[\frac{2 m}{\hbar^{2}}\left(W-E_{F}-\phi+k \frac{e^{2}}{\xi}+e F \xi\right)\right]^{\frac{1}{2}}
\end{gathered}
$$

and $W_{l}$ is related to potential barrier peak: $W_{l} \equiv V_{\max }+\left(1-\frac{1}{\sqrt{2}}\right)\left(e^{3} F\right)^{\frac{1}{2}}$. We note that $\lambda(\xi)$ has a singularity at $\xi=0$. Modinos [3] notes that it does not affect validity of the equation (1.16) for the potential (1.18a) in case of the normal energies $W<V_{\max }$. However for the $W>V_{\max }$ case eq. (1.16) may not be applicable. However, he also notes 
that for $W>W_{l}$ transmission coefficient $D(W, F)$ should be close to the unity when we use eq. (1.21).

It is important to remember that eq (1.21) describes the current density based on free electron model. As it was noted before, this approximation is valid for those quantities which depend only on the height of the surface barrier and its asymptotic behavior $\mathrm{z}>3 \AA$. There are other phenomena related to electron scattering at the metalvacuum interface which cannot be properly described by free-electron model since there is anisotropy associated with crystalline structure of the metal and subsequently its band structure [9].

\subsection{Field Emission}

From the general expression for current density (1.21) it is possible to analytically obtain an expression for specific regions of the temperature and the applied electric field. As a result one can arrive at the expressions for the field or thermionic emissions.

At low temperatures (room temperature) and for typical surface barrier of (most of the metals) $\phi \approx 4-5 \mathrm{eV}$ in the presence of the external electric field $(F \approx 4000 \mathrm{~V} / \mu \mathrm{m})$, the second integral in eq. (1.21) is negligible. The first integral has significant contribution to $J(F, T)$ only in the vicinity of the Fermi level. Therefore, the eq. (1.21) under these conditions will be reduced to the following expression,

$$
J(F, T)=\frac{e m k_{B} T}{2 \hbar^{3} \pi^{2}} \int_{-\infty}^{+\infty} \exp [-Q(W)] \ln \left[1+\exp \left(-\frac{W-E_{F}}{k_{B} T}\right)\right] d W
$$


where, $\exp [-Q(W)] \gg 1$ for the defined conditions for $\phi$ and $F$ values.

The above expression could be further transformed, considering the immediate proximity of the Fermi level (region for which integral is defined) [9] which allows a Taylor expansion of $Q(W)$ around $E_{F}$ and using two first terms of expansion in the exponent in the eq.1.21. Integrating transformed eq. 1.21 and considering very low temperature approximation one can obtain the Fowler - Nordheim equation [9]

$$
J(F)=A_{f e} F^{2} \exp \left(-k \frac{B_{f e} \phi^{\frac{1}{2}}}{F}\right)
$$

where

$$
A_{f e} \equiv \frac{e^{3}}{\left[16 \pi^{2} \hbar \phi t^{2}\left(\frac{\left(k e^{3} F\right)^{\frac{1}{2}}}{\phi}\right)\right]}
$$

and

$$
B_{f e}=\frac{4}{3 e}\left(\frac{2 m}{\hbar^{2}}\right)^{\frac{1}{2}} v\left(\frac{\left(k e^{3} F\right)^{\frac{1}{2}}}{\phi}\right)
$$

and $v, t$ are so called "special field emission elliptic functions" [12].

The Fowler - Nordheim equation (1.22) describes the current density originating from the electron tunneling trough the surface potential barrier where bending and narrowing of the barrier is induced by the external electric field (Figure 1.4).

The strength of the electric field affects the bending of the potential barrier, its thickness and height. Thus an increase of the magnitude of the applied electric field would decrease 
the tunneling distance within the barrier and in result would increase population of the electrons (occupying states close to $\mathrm{E}_{\mathrm{F}}$ ) which tunnel through potential barrier to the vacuum.

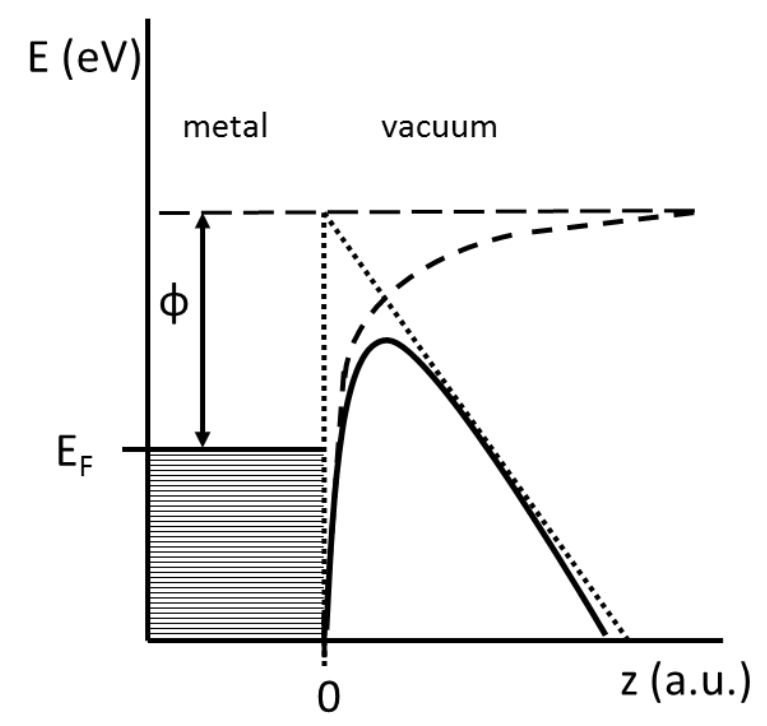

Figure 2.4 Surface potential barrier for field emission as described by the Fowler Nordheim. The surface potential barrier is represented by a solid line. Contribution from the electric field is shown by dotted line and from the image potential - by dashed line.

It is important to note that by plotting $\ln \left(\frac{J}{F^{2}}\right)$ versus $\frac{1}{F}$ one acquires the curve which is practically a straight line, known as the Fowler-Nordheim (FN) plot. The slope of a FN plot according to the Modinos [9]

$$
S_{F N}=\frac{d \ln \left(\frac{J}{F^{2}}\right)}{d\left(\frac{1}{F}\right)}=-\frac{4}{3 e k}\left(\frac{2 m}{\hbar^{2}}\right)^{\frac{1}{2}} S\left(\frac{\left(k e^{3} F\right)^{\frac{1}{2}}}{\phi}\right) \phi^{\frac{3}{2}}
$$

where

$$
s(y)=v(y)-\frac{y}{2} \frac{d v}{d y}
$$


And $\mathrm{s}(\mathrm{y})$ is also a special field-emission elliptic function which is practically constant within a narrow region of the applied field and close to the unity. $S(y)$ like $v(y)$ and $t(y)$ were computed and tabulated. However these elliptical functions are slowly varying with the change of the $F$ and $\phi$. Further, $s(y)$ and $t(y)$ are both close to unity for the typical values of the electric field and work function. When $s(y) \rightarrow 1$ slope from equation (1.23) has the given form

$$
S_{F N}=-\frac{4}{3 e k}\left(\frac{2 m}{\hbar^{2}}\right)^{\frac{1}{2}} \phi^{\frac{3}{2}}
$$

which is the case of the triangular barrier if we neglect image potential term in eq. (1.18a.). According to Modinos, for typical values of $\phi$ and $F$, the proportionality coefficients of the extracted values of the slope $S_{F N}$ and $\phi^{\frac{3}{2}}$ change by about $5 \%$ when the potential barrier (1.18a) is replaced by a triangular barrier. Thus for metals, any realistic barrier should be situated somewhere between these two [9].

Following the main conclusion from the Fowler-Nordheim equation, in order to initiate tunneling through the potential barrier and achieve a desired value of the current density, it is required that the external electric field of specific value for given material. Considering the flat surface of the metal there are required also very high applied fields, which in practice means very high applied voltages. In order to achieve high fields, the phenomena known from classical electrostatics was utilized considering the structure with high aspect ratio, and high curvature at the tip as the emitting surface (cathode) as shown in the Fig. 2.5b. 
a)

\begin{tabular}{|c|c|c|c|c|c|}
\hline & $T$ & $T$ & $\mathbf{T}$ & $\mathbf{T}$ & $T$ \\
\hline & I & I & I & I & I \\
\hline & I & I & I & 1 & I \\
\hline & I & I & I & I & I \\
\hline Electrodes & I & I & I & 1 & 1 \\
\hline & I & I & I & I & I \\
\hline & i & i & I & I & i \\
\hline & I & I & I & I & I \\
\hline & I & I & I & 1 & \\
\hline
\end{tabular}

b)

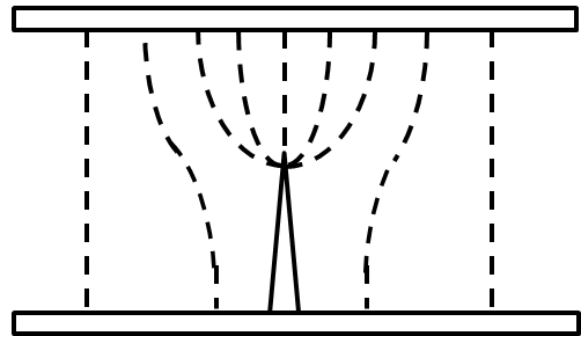

Figure 2.5 The electric field lines represented by dashed lines between two electrodes: a) for uniform field with the two flat electrodes; b) for non-uniform field in the presence of the "sharp" (high aspect ratio) structure on the bottom electrode.

High curvature will result in very high local electric field $F_{l}$ which differs from macroscopic ("applied") field $\mathrm{F}_{a}[5]$. The relation between local and macroscopic fields is defined by dimensionless coefficient,

$$
\beta=\frac{F_{l}}{F_{a}}
$$

The parameter $\beta$ from above equation is called enhancement factor as it is a measure of the ability of the emitter to increase locally (in vicinity of the emitting surface) the magnitude of the applied electric field. Thus considering that in practice $F_{a}$ is defined by the voltage applied to the base of the studied sharp structure (Figure 2.5) we can conclude that the higher is the value of the $\beta$, the lower is magnitude of the applied field required to initiate a tunneling or reach desired value of the current density. Also, depending on the morphology of the substrate and emitter, different expressions of $\beta$ can be obtained empirically and would have similar correlation with aspect ratio $h / r$ (height of structure/radius of the tip curvature) of the emitter [11]. 
For a complete description of the field emission from the structures of more complex geometry, as one shown in the Figure 2.5b, in F-N eq. 1.22, electric field $F \equiv F_{l}=\beta F_{a}$ [7]. Now, modified F-N equation will reflect the effect of the surface morphology of the emitter in the field emission. In expression for the slope of F-N plot for the triangle barrier will appear additional term,

$$
S_{F N}=-\frac{4}{3 e k}\left(\frac{2 m}{\hbar^{2}}\right)^{\frac{1}{2}} \frac{\phi^{\frac{3}{2}}}{\beta}
$$

In practice when conducting experimental study of field emission from given material usually is recorded variation of the current $I$ depended on the applied voltage $V$. For convenience in analysis of the experimental data, a simplified form of the FN equation (1.22) representing $I$ dependence on the applied voltage $V$ [14] is commonly used.

$$
I(V)=a_{F E} V^{2} \exp \left(-\frac{b_{F E} \phi^{\frac{1}{2}}}{\beta V}\right)
$$

where constant $a_{F E}$ contains information on effective emission surface $S$ as well on separation distance $d$ between cathode and anode; $b_{F E}=\frac{4}{3 k e}\left(\frac{2 m}{\hbar^{2}}\right)^{\frac{1}{2}} d$. The argument of the exponent in this version from eq (1.27) is very similar to the one in reduced formula (1.22) for the triangle barrier approximation. The slope obtained by plotting 
$\operatorname{Ln}\left(I / V^{2}\right)$ versus $1 / V$ is not dependent on the $a_{F E}$ constant, thus value of this constant is irrelevant in analysis of the $\beta$.

\subsection{Thermionic Emission}

Thermionic emission is the promotion of electrons to the vacuum from a hot surface of a conducting material. Thus we apply appropriate set of conditions for the general expression of the emission current density (1.21). At high temperatures and weak fields most of the contribution in the integral (1.21) comes from a very narrow region of energy at the top of the surface potential barrier. These conditions allow to substitute in (1.21) for $N(W, T)$ and $D(W, F)$ series expansions of these quantities valid for $W \approx V_{\max }$ [9]. Consequently, the two integrals in eq. (1.21) could be replaced by the following one integral,

$$
J(F, T)=\frac{e m k_{B} T}{2 \hbar^{3} \pi^{2}} e^{-\frac{\phi}{k_{B} T}} \int_{0}^{\infty} \frac{\exp \left(-\frac{W-E_{F}-\phi}{k_{B} T}\right)}{1+\exp \left[-\left(\frac{F \hbar^{4}}{m^{2} e^{5}}\right)^{-\frac{1}{4}} \pi\left(1+\frac{W-E_{F}-\phi}{\left(k e^{3} F\right)^{\frac{1}{2}}}\right)\right]} d W
$$

when appropriate conditions for the thermionic emission are satisfied - as defined by Murphy and Good [11] and Modinos [9]. The above expression is effectively zero except in the neighborhood of the barrier top and the lower limit could be replaced by $-\infty$. The integral could be calculated analytically and the result is 


$$
J(F, T)=\frac{e m\left(k_{B} T\right)^{2}}{2 \hbar^{3} \pi^{2}}\left(\frac{\pi h_{0}}{\sin \pi h_{0}}\right) \exp \left(-\frac{\phi-\left(k e^{3} F\right)^{\frac{1}{2}}}{k_{B} T}\right)
$$

where

$$
h_{0} \equiv\left(\frac{F \hbar^{4}}{m^{2} e^{5}}\right)^{-\frac{1}{4}} \frac{\left(k e^{3} F\right)^{\frac{1}{2}}}{k_{B} T}
$$

For the weak applied electric fields, when $\pi h_{0} \ll 1$ eq. (1.28) reduces to the well-known Schottky formula [9]

$$
J(F, T)=A_{R} T^{2} \exp \left(-\frac{\phi-\left(k e^{3} F\right)^{\frac{1}{2}}}{k_{B} T}\right)
$$

where

$$
A_{R}=\frac{e m k_{B}^{2}}{2 \pi^{2} \hbar^{3}}=120 \frac{A}{c m^{2} K^{2}}
$$

is the so called Richardson constant. Electron emission described by eq. (1.30) is known as field-enhanced thermionic emission. Note that even the application of a weak electric field would cause lowering of the barrier height, as illustrated in figure 2.5 , by the value

$$
\Delta \phi \equiv\left(k e^{3} F\right)^{\frac{1}{2}}
$$




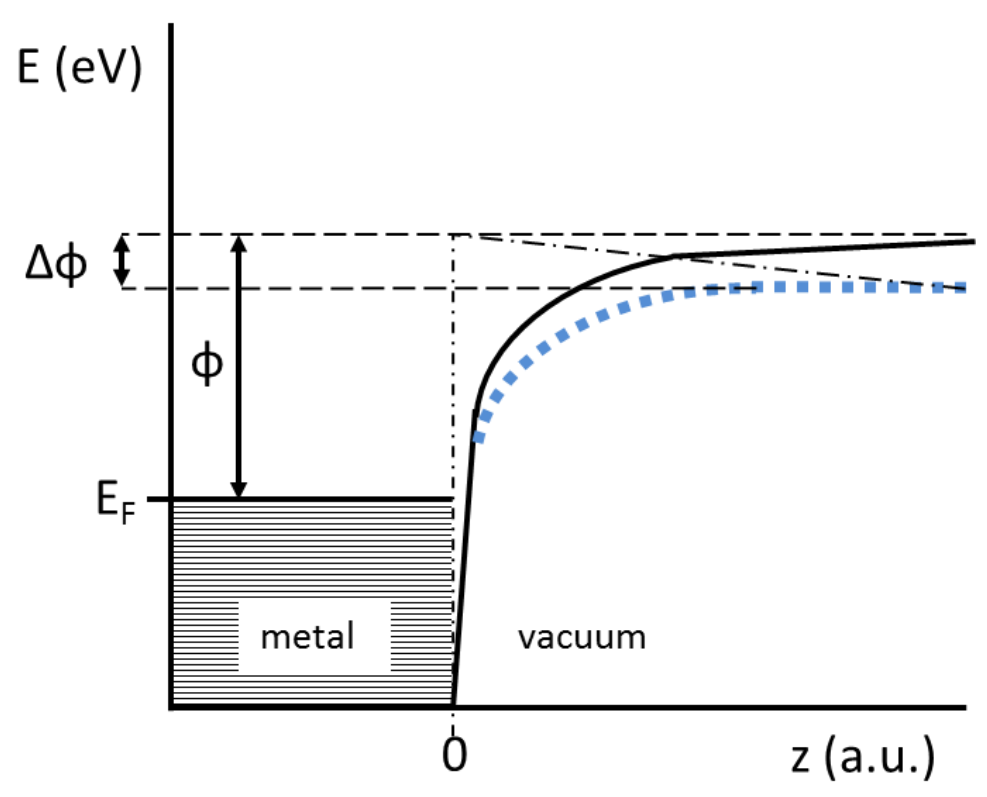

Figure 2.6 Surface potentials for the thermionic emission and Schottky emission (field enhanced TE). Potential in the weak electric field (for Schottky emission) is represented by the blue dotted line. Image potential for thermionic emission is shown as the black solid line and contribution from the field is represented by the dashed-dot line.

In a typical thermionic emission experiment, $\Delta \phi \leq 0.1 \mathrm{eV}$ [9]. Also, the position of the peak of the barrier lies sufficiently far away from the surface $(\gg 4 \AA)$ so that there is no ambiguity considering the validity of the image law in this case.

A more realistic theory of the thermionic emission from metal surfaces leads to the following modified equation,

$$
J(F, T)=t(T, F) A_{R} T^{2} \exp \left(-\frac{\phi-\left(e^{3} F\right)^{\frac{1}{2}}}{k_{B} T}\right)
$$

Where $t(F, T)$ is an average transmission coefficient, which depends on the particular properties of the emitter surface. Furthermore, $t$ depends weakly on the field and 
temperature [9]. If we consider the case without electric field, $F=0$, then eq (1.31) reduces to the known formula,

$$
J_{0}(T)=t_{0} A_{R} T^{2} \exp \left(-\frac{\phi}{k_{B} T}\right)
$$

where $t_{0} \equiv t(0, T)$. The above formula is known as the Richard-Laue-Dushman equation.

According to the (1.31) if we plot $\operatorname{Ln}(J(F, T))$ versus $F^{\frac{1}{2}}$ we would obtain the straight line (so called Schottky line) with the slope $S_{S}=\frac{(k e)^{\frac{3}{2}}}{k_{B} T}$ and intercept $J_{0}$ - so called zero field value of the emission current density. Most of the thermionic emission experiments performed so far from different materials have confirmed the validity of the Schottky theory to a very good degree of approximation [9] - under the condition that space charge effects are neglected.

The zero field value of the emitted current density, which could be obtained from the Schottky lines, corresponds to the quantity defined by the Richardson eq. (1.32). By plotting $\operatorname{Ln}\left(\frac{J_{0}}{T^{2}}\right)$ versus $\frac{1}{T}$ one would obtain a straight line with the slope given by

$$
S_{R}=-\frac{\phi}{k_{B}}
$$


Thus one could determine the value of the work function (from equation (1.32)) by performing linear fit of the thermionic emission experimental data with no need for specific information about $t_{0}$.

Similarly as for the field emission, we can construct the formula which could be used for the analysis of the experimental data. By modifying the Schottky equation (1.31) we obtain a formula for the current $I$ dependence on applied voltage $V$ and temperature $T$

$$
I(V, T)=a_{T E} T^{2} \exp \left(-\frac{\phi-b_{T E} V^{\frac{1}{2}}}{k_{B} T}\right)
$$

where $a_{T E}=t(T, F) A_{R} S(\mathrm{~S}-$ emission area $)$ and $b_{T E}=\left(\frac{k e^{3}}{d}\right)^{\frac{1}{2}}(\mathrm{~d}-$ separation distance). And equation for the zero field current,

$$
I_{0}(T)=a_{T E}^{0} T^{2} \exp \left(-\frac{\phi}{k_{B} T}\right)
$$

where $a_{T E}^{0}=t_{0} A_{R} S$.

As we noted before, the description of the thermionic emission based on free electron model is an approximation and derived formulas are applied to the emission from a plane and uniform metal surface. Nevertheless, experimental thermionic emission studies from various planes of the single crystal tungsten revealed that thermionic emission values of the work functions are in agreement with the values obtained by other methods - such as field emission, photoemission and others [9]. The values of the work 
functions for different planes are presented in Table 1 from the review paper by Riviere $[16]$.

Table 1.1 Best estimate of the measured values of $\phi$ for some faces of Tungsten.

\begin{tabular}{ccccc}
\hline face & 110 & 111 & 116 & 100 \\
\hline$\phi(\mathrm{eV})$ & 5.30 & 4.40 & 4.30 & 4.58 \\
\hline
\end{tabular}

According to Modinos [9] this anisotropy of the work function is related to the presence of electrostatic dipole layer which is a small fraction of the surface potential at the metalvacuum interface [9]. 


\section{CHAPTER 3}

\section{WORK FUNCTION OF CARBON NANOTUBES AND DIAMOND}

In this chapter we review relevant and significant work published by other researchers in relation to the work function of CNT and diamond. Specifically, we concentrate on thermionic emission as the method of determination of $\phi$ and study of the electron emission properties of the carbon materials. Also, we discuss how the intrinsic properties of CNT and diamond affect thermionic emission of these materials.

\subsection{Work function of carbon nanotubes and their thermionic emission properties.}

\subsubsection{Introduction.}

Carbon is an element of the IV group in the periodic table having a half filled valence shell with the electronic configuration $2 \mathrm{~s}^{2} 2 \mathrm{p}_{x}{ }^{1} 2 \mathrm{p}_{y}{ }^{1}-4$ valence electrons. In the case of carbon nanotubes, some $s$ and $p$ orbitals will hybridize to form strong $\mathrm{sp}^{2}$ bonds. Together with their rolled honey comb structure, (Figure 3.1) carbon nanotubes have superior, mechanical, electrical, and thermal properties which make them suitable for many applications, including effective field emitters and thermionic emitters. Carbon 
nanotubes have especially been proven to be a promising material for field emission cathodes. Various configurations based on these materials have been shown to produce high current densities and low turn on voltages $[17,18]$. Carbon nanotubes are known to be mechanically, chemically and electrically robust. In contrast, metal oxides are not suitable for high current emission because they have too-high electrical resistivity and cause serious joule heating, which quickly damages the emitter when operating at
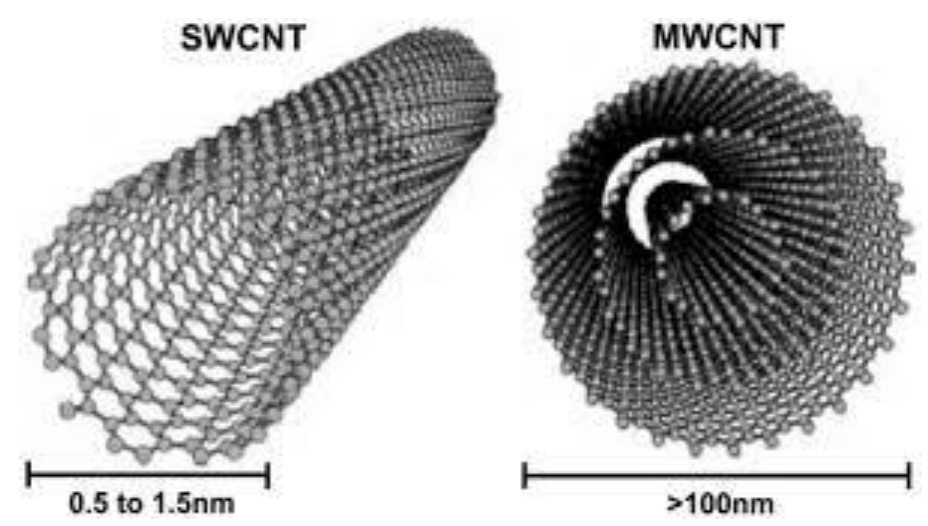

Figure 3.1 Schematic view of the single wall carbon nanotube (SWCNT or SWNT) and multiwall carbon nanotube (MWCNT or MWNT). (source http://galleryhip.com/carbonnanotubes.html, 10/2014).

high current density. It has been shown that poor vacuum conditions do not destroy carbon nanotube emitters, though it lowers their performance [19]. The geometrical properties, such as small tip size and high aspect ratio of carbon nanotubes, are believed to be responsible for the advantage of carbon nanotubes over other conventional materials. As a result, carbon nanotubes are considered to have great potential for field emission and thermionic emission applications.

It is evident that the important parameter that governs the thermionic emissions is the work function, $\phi$. There has been a wealth of results on experimental measurements (by different methods and theoretical calculations of the work function of carbon 
nanotubes [20-36]. We will present some of the reported results on work function values of the carbon nanotubes, first by shortly discussing various methods such as photoelectron emission (PEE), photoemission electron microscopy (PEEM) ultraviolet photoelectron spectroscopy (UPS) and contact potential difference (CPD). Another method having especially practical use in case of the CNT is thermionic emission which we will discuss more in detail.

\subsubsection{Work function of the CNTs - methods of measurement.}

First we will review results of the theoretical work on the work function of the carbon nanotubes reported by a few research groups.

Zhao et al. performed one of the first studies of the work function of single wall carbon nanotubes and SWNT bundles using first-principles methods [32]. Authors considered the models of the semiconducting and metallic tubes and investigated the dependence of $\phi$ on the chirality and tube diameter. Results showed (Table 3.1) that the $\phi$ of individual metallic nanotubes is weakly dependent on the diameter, chirality and is comparable to graphite $\phi$ of $4.91 \mathrm{eV}$. Work function of the semiconductor SWNT decreases with the increase of the tube diameter. Calculations showed, however, that the bundles (ropes) made of SWNT have slightly larger work function than individual SWNT and have no clear dependence on the diameter or chirality. Even though it is not explicitly stated in the work, the object of interest was most probably the side walls of CNT.

Another first-principles study of the CNT work function conducted by Bin Shan and Kyeongjae Cho [33], confirmed that for the tubes with diameters larger than $1 \mathrm{~nm}$ there is no significant dependence on the diameter or chirality, and value of $\phi$ is 
comparable to the one of the graphene sheet $4.66 \mathrm{eV}$ (Figure 3.1). That is not the case for diameters less than $1 \mathrm{~nm}$ - as it could be seen from the results presented in Table 3.1 values could vary from $4.5 \mathrm{eV}$ up to $5.9 \mathrm{eV}$.

Tab 3.1 Theoretical work function values of carbon nanotubes and carbon materials.

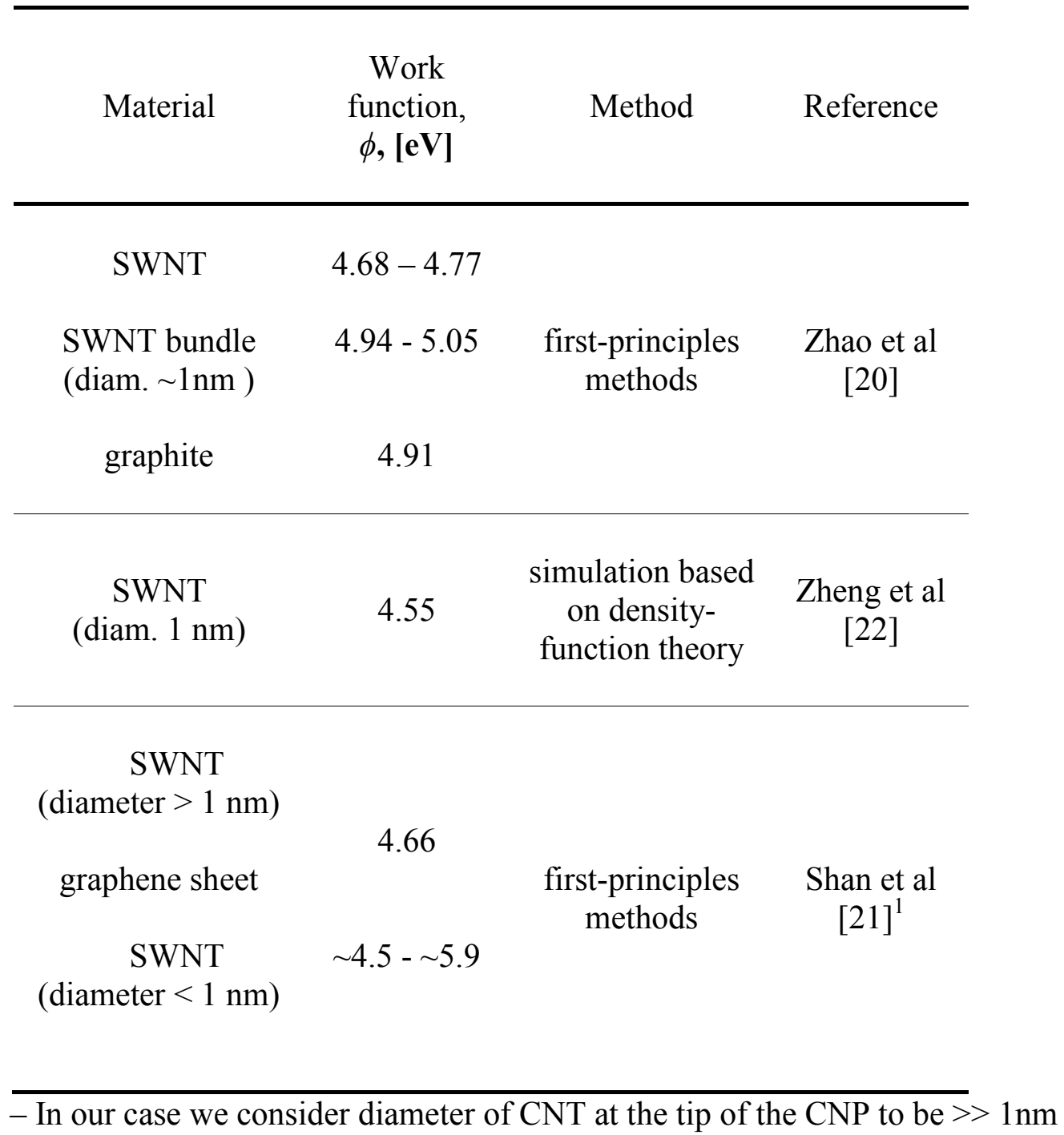

Note that even though it is not explicitly stated in the first principles studies described above, the object of interest were most probably side walls of the CNT in the both cases. Earlier results reported by Zhou et al. regarding the electron structure of the openedended SWNT showed that the presence of non-bonding valence electrons at the tip 
induces variation of the electronic structure and importantly decrease of the work function. Interestingly, one of the other effects is also strengthening of the bonding between the carbon atoms at the mouth of the open - ended CNT [23]. Other theoretical studies by Chen et al. and Zheng et al. of the work function confirmed the fact about lower values of the work function at the tips for the capped and the open-ended SWNT $[22,24]$. On the other hand these studies showed that a decrease of the value of $\phi$ at the capped or open-ended tip depends on the chirality.

To date there are several earlier standard experimental methods for determining the work function of different materials: PEES, PEEM, UPS and CPD. These techniques were used also for the measurements of $\phi$ of the carbon nanotubes.

Photoelectron emission study of the SWNT "bucky paper" and MWNT (grown by arc discharge from the graphite rods) carried out by Shiraishi et al [25] showed that value of the work function of the both single- and multiwall carbon nanotubes, $5.05 \mathrm{eV}$ and $4.95 \mathrm{eV}$ respectively, are larger than for the high oriented pyrolitic graphite (HOPG): $4.80 \mathrm{eV}$ (Table 3.2). It was reported that diameters of the SWNT and MWNT were about $1 \mathrm{~nm}$ and $20 \mathrm{~nm}$ respectively. The higher value of the $\phi$ for CNT compared to HOPG was interpreted as being due to the differences in geometry of the structures. Authors suggest that considering that HOPG (as single graphene sheet) has orthogonal $\sigma-\pi$ valence states. CNT's have mixed $\sigma-\pi$ valence states that will causethe work function to increase. This was supported by additional PEE measurements of the work function of the fullerenes $\mathrm{C}_{60}: 6.0 \mathrm{eV}$; and first principles calculations which reproduced experimental results. In this study, authors determined work function of the sidewalls of the CNT. 
As mentioned earlier, a commonly used experimental method for determination of the work function of different materials is ultraviolet photoelectron spectroscopy. Ago et al. were the first to report the values of the work function of purified MWNT determined by UPS [27].

Tab 3.2. Values of work function of carbon nanotubes and other carbon materials obtained by different experimental methods.

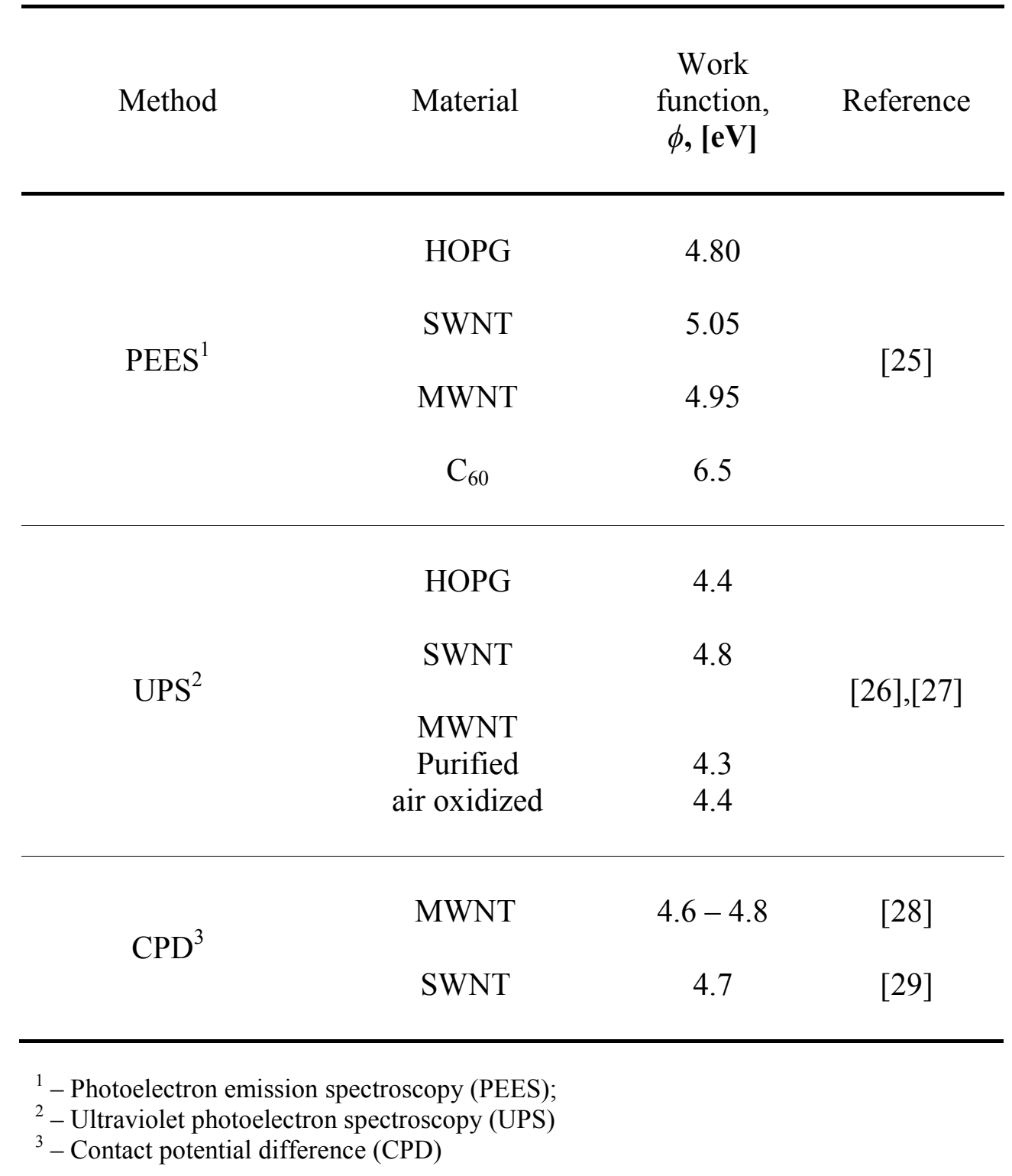


MWNT were grown by arc discharge on the graphite rods (with no catalyst) and were prepared for measurements in a form of the thin film made of metallic and semiconducting MWNT bundles. Measured value of the work function of the MWNT is $4.3 \mathrm{eV}$ and for the HOPG $4.4 \mathrm{eV}$.

Another UPS study by Suzuki et al. revealed that work function of the SWNT (grown by laser ablation) is $4.8 \mathrm{eV}$ and for the graphite $4.6-4.7 \mathrm{eV}$. Tested samples were SWNT bundles where the average diameter of SWNT was $1.4 \mathrm{~nm}$ and that of the bundle $20 \mathrm{~nm}$.

In the second study CNT samples were annealed before the UPS measurement which was not the case for the first one. This could be the source of the discrepancies in the value of the work function between the two groups. The presence of adsorbed molecules of the nitrogen, oxygen or water on the surface of the CNT can affect the values of work function [22]. Considering that in both UPS studies measurements were done on CNT bundles (nonaligned CNT), the spectra are dominated by contribution from the sidewalls of the CNT. Thus authors reported $\phi$ of the sidewalls. It is evident that Suzuki et al. results are in agreement with the theoretical results.

Another method often used for the determination of the work function is Contact Potential Difference. Gao et al. performed experiments on individual MWNT (synthesized by arc discharge) using in situ transmission electron microscope (TEM) technique [29] which is still the CPD method in principle. In this study authors determined that values of the work of the tip of individual MWNTs are in the range 4.6 $4.8 \mathrm{eV}$. Results showed that $\phi$ is not dependent on the diameter $(14-55 \mathrm{~nm})$ of the tubes. Cui et al. CPD measurements of the SWNT [30] resulted in similar values of the work function: $4.6 \mathrm{eV}$. 


\subsubsection{Thermionic emission of the CNT and work function determination.}

Field emission properties of various carbon nanotubes, including single-walled carbon nanotubes (SWNTs), multi-walled carbon nanotubes (MWNTs) [17-19, 36, 37] and conical carbon nanotubes (CCNTs) [40] have been extensively studied. There has been limited work on the thermionic emission of CNT grown by plasma enhanced CVD (PECVD) or standard thermal CVD. Apart from the investigation of the thermionic emission properties of these structures, this type of the measurements was used as a method of determination of the $\phi$ (Table 3.3). The main advantages of the thermionic emission-based method of obtaining work function of the CNT are the (i) accuracy of the measurement and (ii) elimination of the adsorbents [32].

D. Cox et al. were the first to report on the thermionic emission from the individual MWNT (grown by low temperature CVD) [31] using a nanomanipulation system contained within scanning electron microscope (SEM). Individual MWNTs (diameter: $50-100 \mathrm{~nm}$ ) were selected on the substrate and manipulated in situ, in real time, in a SEM with the help of the two sharpened W tips.

According to authors, in spite of the low temperature CVD synthesis, the MWNTs nanotubes were defective and as such had particularly low temperature conductivity. Utilizing this fact and using $\mathrm{W}$ tips as electrodes and running current through the individual nanotube it was possible to significantly increase the temperature, $T$, of the nanotube. Using copper wire for the third electrode and applying voltage, it was possible to measure the thermionic emission (TE) current from the heated MWNT. Figure 3.4 demonstrates the dependence of TE current on heating current which was flowing through the nanotube. It can be seen that this resembles an exponential dependence. Thus, 
authors estimated approximate values of temperature (T) using Richardson-Dushman law (eq. 1.1) and $4.95 \mathrm{eV}$ as the value of the work function of the MWNT sidewall from [18]. According to estimation, $2150 \mathrm{~K}$ is the temperature at which electron emission started; and maximum temperature in this experiment is $2900 \mathrm{~K}$.

Table 3.3 Values of work function of carbon nanotubes obtained from thermionic emission measurements.

\begin{tabular}{|c|c|c|c|}
\hline Material & $T$ range $[\mathrm{K}]$ & $\begin{array}{l}\text { Work function, } \\
\phi[\mathrm{eV}]\end{array}$ & Reference \\
\hline a) Sidewalls & & & \multirow{6}{*}{ [33] } \\
\hline SWNT & $1817-2136$ & $4.70-4.92$ & \\
\hline DWNT & $1846-2151$ & $4.85-4.87$ & \\
\hline MWNT & $1747-2115$ & $4.80-4.91$ & \\
\hline b) Tips & & & \\
\hline MWNT & $1827-2091$ & 4.41 & \\
\hline MWNT yarns & $1468-2207$ & $4.54-4.64$ & [32] \\
\hline $\begin{array}{c}\text { MWNT } \\
\text { (vertically aligned) }\end{array}$ & $1366-1432$ & 4.2 & [35] \\
\hline MWNT yarns & $1100-2300$ & $4.60-4.62$ & [36] \\
\hline MWNT sheets & & $4.55-4.62$ & \\
\hline $\begin{array}{c}\text { MWNT } \\
\text { (vertically aligned) }\end{array}$ & $2021-2398$ & 4.5 & [37] \\
\hline
\end{tabular}


Alternative proper determination of the temperature of the nanotube applying heat transfer analysis based on the dissipative self-heating could not have been possible due to difficulties with accurate value of the thermal conductivity for the MWNT. On the other hand, use of the Richardson - Dushman equation 1.1 could not have provided accurate estimation of the temperature. Because of the fact previously discussed in chapter 2, that the value of the Richardson constant $A_{0}$ from simplified R-D eq. 1.1 is not applicable even in the case of the clean smooth surface of the real metals $\left[25,2^{\text {nd }}\right.$ chapter $]$ - even more it is not applicable in the case of the MWNT. Nevertheless, the exponential part of eq 1.1 or eq. 2.32 is still valid which is illustrated by experimental data [32].

A thorough description of the thermionic emission from the CNT bundles and yarns as well as determination of the value of the work function was presented by Peng Liu et al. $[32,33]$.

The values of $\phi$ obtained by thermionic emission method [32,33] (Table 3.3) are consistent with the theoretical predictions [20-22] (Table 3.1) and roughly agree with photoelectron emission (PEES) measurements [25] (Table 3.2).

Initially, Peng Liu and colleagues investigated thermionic emission from the MWNT yarns. Authors had developed a method to prepare super aligned MWNT arrays by PECVD and from which continuous pure MWNT yarns can be directly drawn. For measurements, samples were prepared using $2 \mathrm{~cm}$ long yarns with diameter of 20-30 microns with ends attached to the electrodes. An Mo plate served as the anode as was placed in front of the angle shaped MWNT yarn, which served as cathode - this is reffered to as simple vauum diode configuration ([25] (ch. 2, page 37), [18]). The yarn was uniformly heated by passing a current through it and anode voltage was applied 
using a sourcemeter. Measurements were performed in high vacuum at the base pressure $\sim 4 \cdot 10^{-8}$ torr and for temperatures in the range $1468 \mathrm{~K}-2207 \mathrm{~K}$.

By collecting $I-V$ curves for different temperatures and using Schottky eq 2.32 or 2.34 for linear fitting of the accelerating field regime part of the $L n I-V^{1 / 2}$ curves [32] as described in Chapter 2.5, authors determined the values of zero field emission current $I_{0}$ for appropriate temperatures $T$. Then after performing linear fitting of the $\operatorname{Ln}\left(\frac{I_{0}}{T^{2}}\right)$ vs $\frac{1}{T}$, value of the work function of the yarn was determined.

Results of the study by Peng Liu et al. showed that values of the work function vary slightly from sample to sample (Table 3.3). Authors note that electrons are emitted mainly from the sidewalls of the MWNT in the yarn. MWNT tips contribution in the work function value is negligible due to comparably small area (10 $\mathrm{nm}$ diameter). Determined values of the emission constant $A=A_{R} t_{0}$ from eq. 2.32 differed from sample to sample and were much more larger than fundamental Richardson constant: $228-824$ $\mathrm{A} / \mathrm{cm}^{2} \mathrm{~K}$. A possible reason suggested is due to the inaccurate estimation of the emission area due to errors in considering roughness of the yarn surface.

After investigating thermionic emission of MWNT yarns Peng Liu et al. followed with the next study. This time the objects of interest were sidewalls and tips of the CNT, thus bundles of the single-, double- (DWNT) and multiwall carbon nanotubes [33] were prepared for measurement. CNTs were synthesized by CVD on a silicon wafer with patterned catalyst islands. Control of the synthesis allowed controlling the number of the ends (tips) of the nanotubes along the bundle. The measurements were performed using the same method as in the previous study of the MWNT yarns.. Again, CNTs were heated 
by passing a current through it. Experimental procedure and conditions were similar to the one in the previous study.

Results revealed that values of the work function of the SWNT, DWNT and MWNT bundles vary slightly from sample to sample (Table 3.3) in the range $4.7 \mathrm{eV}-$ 4.92eV (Table 33.3), and there is no clear evidence of the dependence on the diameter or number of the walls. The value of work function for tips obtained from measurements was smaller $(4.41 \mathrm{eV})$ than in the case of the sidewalls [33] of the CNT, which is in agreement with theoretical predictions $[23,24]$ and what we discussed at the beginning of this chapter. Due to structural differences between sidewall and tip of the CNT, the electron density of states (DOS) would be different for either case. That will affect the value of the work function which could be lower at the end of the CNT [7.8]. Thermionic emission results of Peng Liu and colleagues confirm this.

In parallel to Peng Liu's work on the MWNT yarns, thermionic emission study of the vertically aligned MWNT (pristine and coated by $\mathrm{BaO} / \mathrm{SrO}$ ) was published by Feng Jin et al [135]. MWNT arrays were synthesized by PECVD on the W ribbon with a thin layer of Ni used as a catalyst. The grown MWNTs were approximately $10 \mu \mathrm{m}$ in length and $200 \mathrm{~nm}$ in diameter. $\mathrm{BaO} / \mathrm{SrO}$ was used to reduce work function of the CNT. However the primary interest is in pristine MWNT. Field and thermionic emission measurements were performed using simple planar diode arrangement [25 (ch2, p.37), 134] in ultra-high vacuum (no details reported on the value of the base pressure) with $\mathrm{W}$ strip with the MWNT array acted as the cathode. A source-meter was used to supply a high voltage across the cathode and anode and to measure the electron emission current flowing from cathode to anode. It was not specified in the article how the sample was heated. A quite large value of the field enhancement factor $\beta=1404$ (for the pristine 
MWNT) was determined from field emission measurements using simplified FowlerNordheim eq.2.22 and eq. 2.26. Thermionic emission measurements were performed using the same method as Peng Liu and colleagues, collecting $I-V$ curves at different temperatures in the range: $1366 \mathrm{~K}-1432 \mathrm{~K}$. The value of the work function obtained from the thermionic emission and Richardson-Dushman equation was $4.2 \mathrm{eV}$ which is lower than in case of the Peng Liu's et al measurements (4.41 eV for tips).

The lower value of work function could be explained using a similar argument as previously, by the fact that for vertically aligned MWNT, emission would occur from the tips. Again in according to the theoretical predictions $[23,24]$ one could expect lower value of the work function. As we will see later, another additional factor could cause the further decrease of the work function at the end of the CNT.

Apart from these basic publications on thermionic emission from CNT there have been few other reports. MWNT sheets and "shrunk" yarns were studied by Yang Wei et al [36]. The experimental set up and procedure are very similar to the one used by Peng Liu et al. Measured values of the work function for yarns and sheets (Table 333) are roughly in agreement with the reported by Peng Liu and colleagues [32]. In this work calculated values of the emission constant for MWNT yarns and sheets are much closer to the Richardson constant. Authors claim that this is due to the structural properties of the samples and more accurate estimation of the emission by utilizing energy conservation law and Stefan-Boltzman law.

Another report by the Kolekar et al [37] on thermionic emission from the vertically aligned MWNT grown by the Water Assisted CVD (super growth) on W wires. W wires with MWNT rolled in a shape of the coil acted as a cathode and a Mo foil was used as the anode. Experimental set up and procedure were similar to the one used by 
Peng Liu et al. Work function determined for such structure was $4.5 \mathrm{eV}$ which is in agreement with other reports on TE from the tips or vertically aligned CNT [32,33].

Finally, we would like to note that chronologically the earliest thermionic emission results from random films of SWNT and MWNT (purified nanotube paper) showed unusually low values of work function for CNT: $1.2 \mathrm{eV}$ [23] and $3.46 \mathrm{eV}$ [24]. However these values are not in agreement with theoretical predictions or majority of the experimental results obtained by other methods [25-29].

\subsection{Work function of diamond and thermionic emission properties of the diamond films.}

\subsubsection{Introduction.}

Diamond is another allotrope of carbon, in addition to carbon nanotubes, with exceptional intrinsic properties as well. In the case of diamond, all four $s$ and $p$ orbitals of the carbon will hybridize to form strong $\mathrm{sp}^{3}$ bonds with tetrahedral configuration (Figure 3.9 a). Combined with crystalline structure of the diamond (Figure $3.9 \mathrm{bc}$ ) they lead to, as we mentioned earlier, unique features which include high mechanical hardness, high carrier mobility, high thermal conductivity, low coefficient of thermal expansion, high breakdown electric field. 
a)

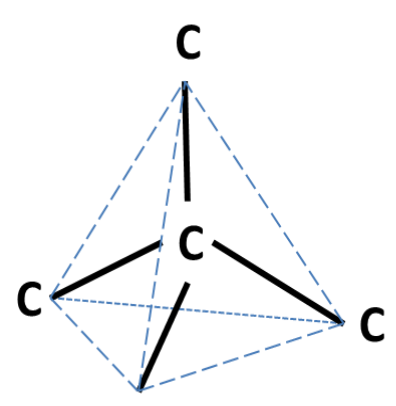

C b)

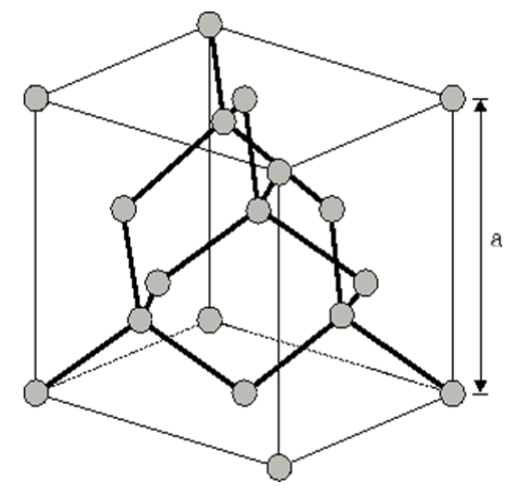

c)

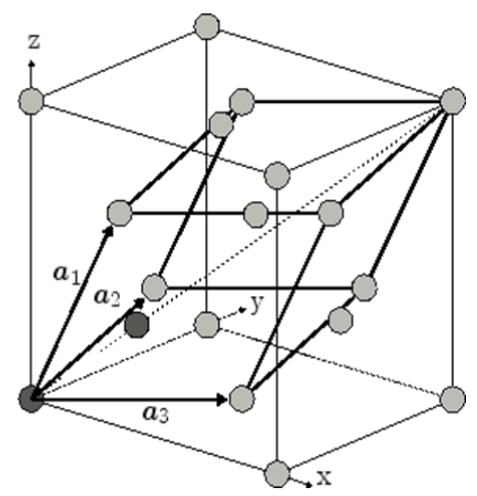

Figure 3.2 a) Schematic view of the tetrahedral $\mathrm{sp}^{3}$ component of diamond unit cell; b) crystallographic unit cell (unit cube) of the diamond structure (a - cell constant, grey circles represent $\mathrm{C}$ atoms); (b) the primitive basis vectors $\left(\overrightarrow{a_{1}}, \overrightarrow{a_{2}}, \overrightarrow{a_{3}}\right)$ of the face centered cubic (fcc) lattice and the two atoms forming the basis are highlighted (Courtesy of Enzo Ungersbock, dissertation, http://www.iue.tuwien.ac.at/phd/ungersboeck/node27.html, 10/2014).

These characteristic features make diamond a good candidate for vacuum electronics and especially for thermionic emission applications. Due to specific properties of diamond, work function (important parameter in TE) could be lowered by: 1) Surface modification/surface coatings of elements inducing dipole moments, affecting the electron affinity; 2) Doping with appropriate dopants causing a change in the position of the Fermi level. What is more, considering the low concentration of intrinsic charge carriers at temperatures below $1000{ }^{\circ} \mathrm{C}$ and a wide band gap $5.47 \mathrm{eV}$ [40], n- type doping would increase conductivity of the diamond what would be beneficial for the electron emission.

Electronic properties of the diamond surface in case of the simplest interface with vacuum are naturally the key factor when discussing the thermionic emission from this material as in case of any semiconductor. Among all the semiconductors, however, diamond possesses a unique feature - the presence of the true Negative Electron Affinity 
of hydrogen terminated surfaces - which we will discuss shortly. Another important subject is the surface and surface defect states in case of the diamond which will be reviewed as well.

\subsubsection{Negative Electron Affinity, $n$ type doping of diamond and Thermionic}

\section{Emission.}

Diamond grown using chemical vapor deposition technique exhibits negative electron affinity (NEA) i.e., the presence of a vacuum level below the conduction band minimum $(\mathrm{CBM})$ due to hydrogen termination on the surface. This can ease the emission of electrons coming

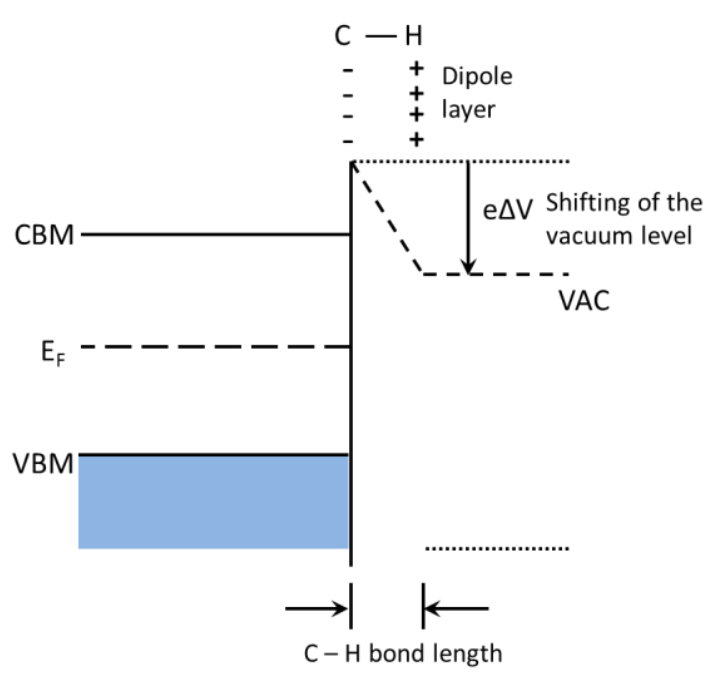
from the conduction band overcoming the small energy barrier that results from the Figure 3.3 Lowering of electron affinity of diamond by electrostatic effect of the surface termination by hydrogen.

reduced work function [41].

On diamond surfaces terminated by hydrogen is formed a surface dipole layer setup by partially ionic $\mathrm{C}-\mathrm{H}$ bonds - the more electronegative carbon is pulling electron from the hydrogen. Presence of such a dipole layer causes a potential step $\Delta \mathrm{V}$ perpendicular to the surface - as it is illustrated in the figure 3.3 [4.2]. This potential step, over a distance of the order of the $\mathrm{C}-\mathrm{H}$ bond length, implies that vacuum level (Vac) position is below the conduction band minimum (CBM). Respectively for fully hydrogen 
terminated surface Vac is lowered by $e \Delta V$ (where $e$ is the elementary charge) compared to its value for clean diamond surface with no dipole layer.

Table 3.4 Experimental values of the electron affinity for different diamond faces.

\begin{tabular}{|c|c|c|c|}
\hline Orientation & Termination & $\begin{array}{l}\text { Electron } \\
\text { aff. [eV] }\end{array}$ & Publication \\
\hline$(100)-(2 \times 1)$ & clean & 0.5 & \multirow{3}{*}{$\begin{array}{l}\text { F. Maier et al, Phys. Rev. B } 64 \text { (2001) } \\
165411\end{array}$} \\
\hline$(100)-(2 \times 1)$ & $\mathrm{H}$ & -1.3 & \\
\hline$(100)-(1 \times 1)$ & $\mathrm{O}$ & 1.7 & \\
\hline$(100)-(1 \times 1)$ & $\mathrm{O}$ & 0.54 & $\begin{array}{l}\text { Y.M. Wang et al, Diam. Relat. Mater. } 9 \\
(2000) 1582\end{array}$ \\
\hline$(111)-(2 \times 1)$ & clean & 0.38 & J. B. Cui et al, Phys. Rev. Lett. 81 (1998) \\
\hline$(111)-(1 \times 1)$ & $\mathrm{H}$ & -1.27 & 429 \\
\hline$(110)-(1 \times 1)$ & clean & 0.7 & $\begin{array}{l}\text { P. K. Baumann and R. J. Nemanich, } \\
\text { Phys. Rev. B 58 (1998) } 1643\end{array}$ \\
\hline$(110)-(1 \times 1)$ & $\mathrm{H}$ & -1.0 & $\begin{array}{l}\text { L. Diederich et al, Surf. Sci. } 424 \text { L314 } \\
\text { (1999) }\end{array}$ \\
\hline
\end{tabular}

Experimental studies revealed that depending on orientation of diamond surface (hydrogenated by plasma), value of NEA vary (Table 3.4) from $-0.7 \mathrm{eV}$ to $-1.3 \mathrm{eV}$ [42]. This is in comparison to oxygen terminated diamond surfaces with value of positive electron affinity ranging from $0.54 \mathrm{eV}$ to $1.7 \mathrm{eV}$ and to the clean diamond surfaces with $\chi$ $=0.38-0.70 \mathrm{eV}[42,43]$.

In addition to the presence of a NEA, it was presumed that the work function of diamond could be further lowered by appropriate $\mathrm{n}$ type doping to bring Fermi level $\left(\mathrm{E}_{\mathrm{F}}\right)$ closer to the conduction band minimum (CBM). We need to note that experimental study of the work function of the nitrogen doped single crystalline diamond and n-type diamond films has shown that situation is more complex. As a result of band bending at the surface, reduction of the work function has not been observed at all or has been less effective than expected. P-type doping into diamond with boron (B) has been widely studied and it is known to introduce an acceptor level $(0.37 \mathrm{eV})$ above the valance band 
maximum [44]. However, study of n-type doping into diamond is limited and has been very challenging due to limited or no incorporation of dopants in to bulk diamond lattice. Several n-type dopants such as nitrogen $(\mathrm{N})$, phosphorus $(\mathrm{P})$, and sulfur $(\mathrm{S})$ have been studied earlier [45] (Figure 3.4).

Nitrogen has been shown to create deep donor levels at $1.7 \mathrm{eV}$ below the bottom of conduction band due to its structural distortion in diamond lattice. Consequently, Ndoped diamond does not yield room temperature conductivity, further limiting its electronic feasibility [46]. Moreover it has been reported that the

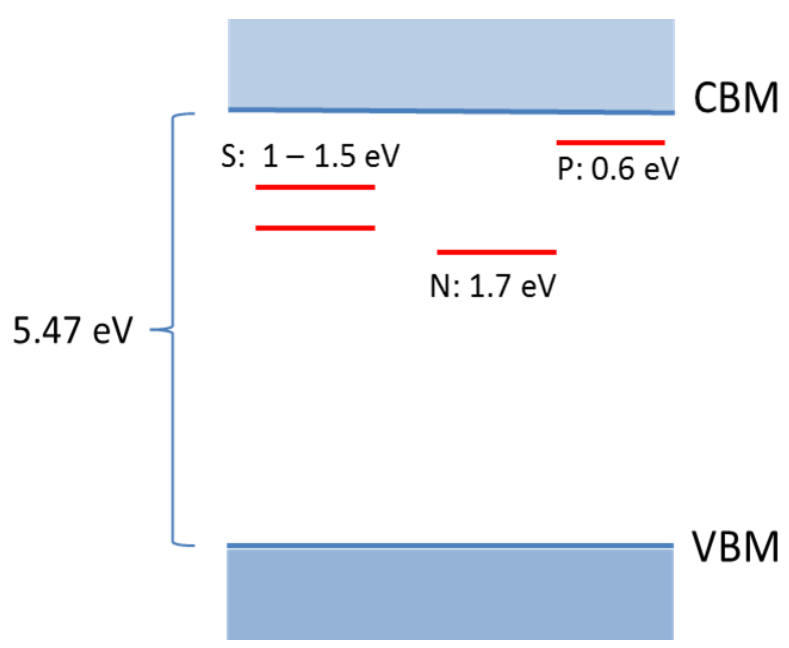

Figure 3.4 Donor levels of the different impurities for the diamond. Where CBM conduction band minimum; VBM - valence band maximum.

n-type conductivity of $\mathrm{N}$ doped polycrystalline/ultra-nanocrystalline diamond (UNCD) films could be attributed to the nitrogen incorporation into grain boundaries (possibly an enhanced conductivity through interconnected shells of grain boundaries) [46]. Though theoretical modeling of sulfur (S) doping into diamond has not been reported to exhibit shallow donor levels, it is still a controversy over the type of donor level (shallow or deep) created by S [45].

Co-doping of sulfur and boron has shown to exhibit n-type conductivity, but no significant reduction in the work function has been observed [47].Doping with phosphorus into single crystalline diamond (111) is known to create a shallow donor level $0.6 \mathrm{eV}$ below the CBM [48]. It is also predicted that in P-doped diamond (polycrystalline or homoepitaxial) films at the back contact (diamond/ cathode metal) 
electrons can be easily extracted due to the presence of ionized donors in the space charge region [49] and due to the creation of defects during P incorporation, which in turn eases the movement of electrons to be extracted [11]. However, the following factors have limited the success of controlled doping of phosphorus into the diamond lattice to any satisfactory level. First, because the size of the P atom is larger than carbon, some native defects and structural displacement will accompany during phosphorus incorporation (lattice mismatch and covalent bond length). Second, P incorporation is more favorable on (111) surface compared to (100) surface [50]. Nevertheless, considerable progress in phosphorus doped n-type diamond has been reported on (111) and (001) orientation [48, 51-53] along with recent study on (100) oriented single crystal diamond [51] using gas phase source of phosphorus. Recently, the growth and incorporation efficiency of phosphorus as a function of surface orientation of grains in [110]-textured polycrystalline CVD diamond has been reported [54]. Growth of phosphorus-doped polycrystalline diamond films on silicon substrates has also been reported using gas phase (as dopant phosphine $\mathrm{PH}_{3}$ ) [55] and organic precursors in case of the single crystalline homoepitaxial P doped films grown on diamond [56]. However, thermionic emission or work function measurements have not been performed in most of these materials.

Some of the investigations based on thermionic emission from nitrogen doped homoepitaxial diamond [57], nanocrystalline (NCD) [58] and ultrananocrystalline diamond (UNCD) [59] films have resulted in observed work functions as low as $1.3 \mathrm{eV}$ (Table 3.5). In other instances, thermionic emission energy distribution (TEED) results on undoped diamond films have been shown to exhibit work function value of $3.3 \mathrm{eV}$ 
[60]. A work function value as low as $0.9 \mathrm{eV}$ has been observed for the phosphorus doped diamond film [61].

Table 3.5 Values of work function of doped diamond films.

\begin{tabular}{cccccc}
\hline Dopants & $\begin{array}{c}\text { Work } \\
\text { function } \\
\varphi[\mathrm{eV}]\end{array}$ & $\mathrm{A}$ & $\begin{array}{c}\text { Type of } \\
{\left[\mu \mathrm{A} / \mathrm{cm}^{2} \mathrm{~K}^{2}\right]}\end{array}$ & $\begin{array}{c}\text { Minimum } \\
\text { diamond } \\
\text { film }\end{array}$ & $\begin{array}{c}\mathrm{T}[\mathrm{K}] \\
\text { References }\end{array}$ \\
\hline $\mathrm{S}$ & $\sim 2.5$ & $40-45$ & $\mathrm{NCD}$ & 880 & \\
\hline $\mathrm{N}$ & $1.5-1.9$ & $0.1-10$ & $\mathrm{NCD}$ & 520 & \\
\hline $\mathrm{P}$ & 0.9 & 10 & $\mathrm{PCD}$ & 620 & \\
\hline
\end{tabular}

Based on the published results of thermionic emission from the $\mathrm{n}$ type doped diamond, it could be speculated that it could be possible in future to tune work function of the diamond by using appropriate dopants in the range of the $\phi$ values $0.9 \mathrm{eV}-2.5 \mathrm{eV}$ which are of significant importance regarding thermionic energy conversion.

Some of the surface modifications/coatings have been reported to lower the work function values of diamond [63-68]. However, thermal stability of these surfaces has either been not tested or unsatisfactory towards the thermionic emission. Hence, the search for the proper material with required work function values is still ongoing.

\subsubsection{Electronic properties of the surface of the diamond. Intrinsic and defect states at the surface of the diamond.}

Surface states are intrinsic features of the material specific for the crystallographic surface under consideration, which presence is the result of the truncation of the periodic 
potential of a crystal by surface - even in the case of ideal perfectly ordered surface of the single crystal $[8,42]$. Surfaces states are the basis for the two dimensional structure which is a display of the dispersion relations between the energy of the corresponding Eigen-states and the crystal momentum vector $\mathbf{k}_{\|}$parallel to the surface. Due to the fact that the crystal lattice unit cell at the surface and near surface is different from the one for the bulk - with the larger surface of unit cell usually [42] (reconstruction phenomenon) dispersion relation of the surface states is determined for the Brillouin zone corresponding to the reconstructed surface $[8,42]$. ]. If the surface states are degenerate with Bloch states [8] of the infinite crystal (with the same $\mathbf{k}_{\|}$), they usually mix and form resonances. In the opposite case, surface states are localized at the surface and can take part in charge exchange with states of semi-infinite bulk but on condition that surface states position is within fundamental energy gap of the semiconductor - for example diamond. In this case surface states could pin the Fermi level at the surface [42].

Surface states on diamond were previously investigated experimentally (electron scattering and spectroscopy) and theoretically (density functional theory) for three reconstructed crystallographic surfaces (111), (110) and (100) and these studies provided a consistent description of the surface states of the main crystallographic surfaces [42]. For all three crystallographic orientations, the dangling bonds of the clean surfaces form $\pi$ - electron systems which lead to different densities of surface states depending on the topology of the surfaces. On diamond reconstructed surface (100) $2 x 1$ atoms form $\pi$ bonded symmetric dimers and based on simple tight binding description this interaction is mainly responsible for the bonding-antibonding $\left(\pi-\pi^{*}\right)$ splitting between occupied and unoccupied (dimer) $\pi$ orbitals. The dimer arrangement on the surface (rows separated by "large" distance of $2.52 \AA$ along (011) direction causes further weaker splitting of the 
dimer orbitals and the formation of the surface band structure with an occupied band originating from the $\pi$ (bonding) orbital and an empty band originating from the $\pi^{*}$ (antibonding) orbital with a gap $1.3 \mathrm{eV}$ between occupied and unoccupied states. It is important to note that occupied surface states do not extend into the absolute gap of the diamond. Instead, they are placed within the valence band with respect to energy, thus no charge exchange could take place between them and bulk diamond and they will not induce band bending [42].

For the surfaces (111) and (110) situation is different. In this case dangling bonds are arranged in symmetric $\pi$ bonded chains without dimerization .

Reconstruction $2 \times 1$ is required on the (111) surface but not for the (110). The distance between $\pi$-orbitals is $1.45 \AA$ for the (111) and $1.54 \AA$ for the $(110)-$ much smaller than for the (100) surface. In result the $\pi$ - state system is formed, which is delocalized in one direction along the chains. As a consequence, these surfaces are predicted to be metallic. Theoretical predictions for the surfaces (111) and (110) are however, in contradiction with the experimental data [42]. For the surface (111) presence of the narrow band gap was detected $(0.5 \mathrm{eV})$ and for (110) there is no conclusive experimental results. There is no clear evidence that surface for these orientations could induce Fermi level pinning at the surface [42].

In case of the hydrogen termination of the surfaces no occupied states within band gap are predicted theoretically or detected experimentally. Unoccupied states are predicted but their presence is not confirmed experimentally [42].

Another type of states localized at the surface are defect states. The most basic surface defect is an isolated dangling bond but these bonds could be arranged along the surface steps or in more or less extended clusters on the surface of the material, not only 
single crystalline but also polycrystalline as well. For diamond behavior of this type of defects approaches asymptotically that of graphitic or more generally $\mathrm{sp}^{2}$ bonded patches.

Two dimensional surface density

of states S-DOS is the important quantity in the discussion of the interaction of the surface defect states with bulk [42]. SDOS is formed by interaction and disorder of the individual defects on the surface of the diamond. Ristein et al. based on the case of the $p$ type diamond

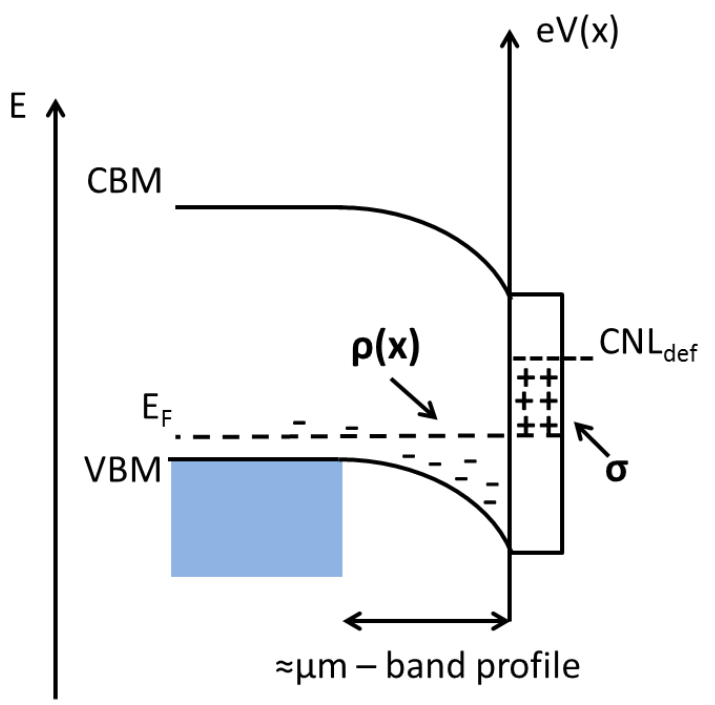
and S-DOS similar to the graphite explained the mechanism of this interaction. In equilibrium the Fermi levels at the interface of the bulk and Figure 3.5 Illustration of defect induced surface band bending on a p-type diamond according to J.Ristein et al., [42]. A graphite like DOS of the surface is assumed.

surface of the diamond need to be aligned. Thus, initially electrons are diffusing from defect states into the bulk of the diamond creating hole depletion layer with negative space charge and positive surface charge density until equilibrium is reached and bulk surface Fermi level is aligned across interface Figure 3.5 [42]. The space charge profile creates the band bending which fulfills Poisson's equation and has to be consistent with the local Fermi occupation statistics and this depends on the local position of the bands relative to Fermi level. It is important to note that under assumption that space charge density $\rho$ in the depletion layer is constant (Schottky approximation) the total area charge density $\sigma$ in the hole depletion area (Figure 3.5) is dependent on acceptor concentration and most importantly on difference between Fermi levels of the bulk and surface. The 
total (negative) charge in the hole depletion layer of the diamond (Figure 3.5) needs to be compensated by the same amount of positive charge in the surface defects. This positive charge is also a function of the surface Fermi level and it is equal to zero when it is aligned with charge neutrality level of the defect system $\mathrm{CNL}_{\text {def. }} \mathrm{CNL}_{\text {def }}$ is assumed to be aligned with intrinsic graphite Fermi level.

According to J.Ristein et al., it is a self-consistent mechanism which allows determination of surface $\mathrm{E}_{\mathrm{F}}$ position and the band profile unambiguously - based on analysis of the relation between the defect system and the band structure and requirement for the total charge neutrality. Essentially, it is a characteristic of the heterosystem similar to the band offset between different semiconductors [42]. Finally, Ristein et al. noted that surface band bending of the moderately doped diamond is effectively induced already by surface defect concentrations of less than $1 \%$ of a monolayer and a $10 \%$ of a monolayer are already sufficient to pin the surface Fermi level. The experimental results of the $\mathrm{X}$ ray photoelectron (XPS) and photoelectron yield spectroscopies of the clean and hydrogenated surfaces (111) and (100) of the single crystal diamond [42] were consistent with presented mechanism.

Independent investigation of the band bending by Diederich et al., has demonstrated that electron affinity and work function of differently terminated, doped (nitrogen and boron) and oriented single crystal diamond surfaces by XPS and ultraviolet photoelectron spectroscopy [43] showed consistency with the surface defect mechanism proposed by Ristein et al. UPS measurements allowed to determine the value of the work function (vacuum level - Femi level energy difference) and presence of the negative electron affinity (conduction band minimum - Fermi level energy difference); XPS 
measurements provided information about value of the band bending at the surface of the doped diamond (shifting of the core carbon 1s levels) [Diederich et al., 43].

Analysis of the XPS and UPS measurements of the (100) surfaces of the strongly Nitrogen doped and Boron doped diamond revealed significant downward band bending for the $\mathrm{N}$ doped diamond in case of the clean $(2.4 \mathrm{eV})$ and hydrogenated surface $(1.7 \mathrm{eV})$.

Experimental results showed that there is evidence for significant downward bending $(1.6 \mathrm{eV})$ for the clean surface of the $\mathrm{B}$ doped diamond; weak band bending $(0.7$ $\mathrm{eV}$ ) was reported for the $\mathrm{H}$ terminated surface.

Again we need to note that this result for the B doped diamond is in good agreement with the experimental results of Ristein et al and what is especially important is consistent with the mechanism proposed by Ristein regarding $p$ type diamond [42]. Thus it is reasonable to apply Ristein's description of the doped diamond-vacuum interface for the $\mathrm{n}$ type diamond.

Kono et al.'s investigations of hydrogen terminated phosphorus doped diamond surface (111) using XPS, UPS and He-I excited secondary electron spectroscopy (SES) showed an upward band bending of $\sim 3.2 \mathrm{eV}$ toward the surface. [53] Surface Fermi level position was determined to be $1.8 \mathrm{eV}$ above valence band maximum. They suggested that such large upward band bending could have been induced by the surface Fermi level pinning caused by surface defect states which behave like graphitic patches in analogous to the case of the $\mathrm{p}$ type diamond studied by Ristein et al. 


\subsection{Summary}

We reviewed different experimental methods of determining the value of the work function of the CNT. It is clear that thermionic emission is an accurate and reliable method considering its consistency with theoretical prediction of the work function and other experimental methods such as PEES. On the other hand, TE measurements could serve as a tool to study surface effects of the pristine or functionalized CNT and of course diamond (doped and with functionalized surfaces).

Previous theoretical and experimental studies show that work function is naturally affected by the specific geometric, atomic and electronic structure of the tips of the CNT. Finally, it has to be noted, that special atomic and electronic properties of the diamond surface indicate that nature of the electron emission (photoemission or thermionic emission) mechanism from this material is not trivial and requires caution in description

of the emission, calculation and/or determination the origins of the value of the work function, . 


\section{CHAPTER 4}

\section{CONICAL CARBON NANOTUBES. THERMIONIC EMISSION PROPERTIES AND WORK FUNCTION}

In this chapter we report results of the study of the thermionic emission properties of conical carbon nanotubes directly grown on platinum wires and graphite foils. The thermionic emission results were used to extract the work function values of CCNTs and further confirmed from ultra-violet emission spectroscopy.

\subsection{Synthesis of the Conical Carbon Nanotubes.}

\subsubsection{Introduction}

Conical carbon nanotube (CCNT) could be considered as modified multiwall carbon nanotubes consisting of central MWNT at the core surrounded by helical graphitic sheets in such manner that it has a conical geometry (Figure 4.1). Due to the unique features CCNTs are naturally expected to have superior field emission characteristics which were experimentally confirmed [69].

CCNTs could be grown on different substrates: Pt wire, graphite foil, W or Mo foil $[69,70]$. Depending on the substrate and synthesis conditions CCNTs have a diverse 
morphology with length of the tube in the range $1-70 \mu \mathrm{m}$, diameter of the tip: $5-100$ $\mathrm{nm}$, and diameter of the base: $0.1-5 \mu \mathrm{m}$ (Figure 4.1).

a)

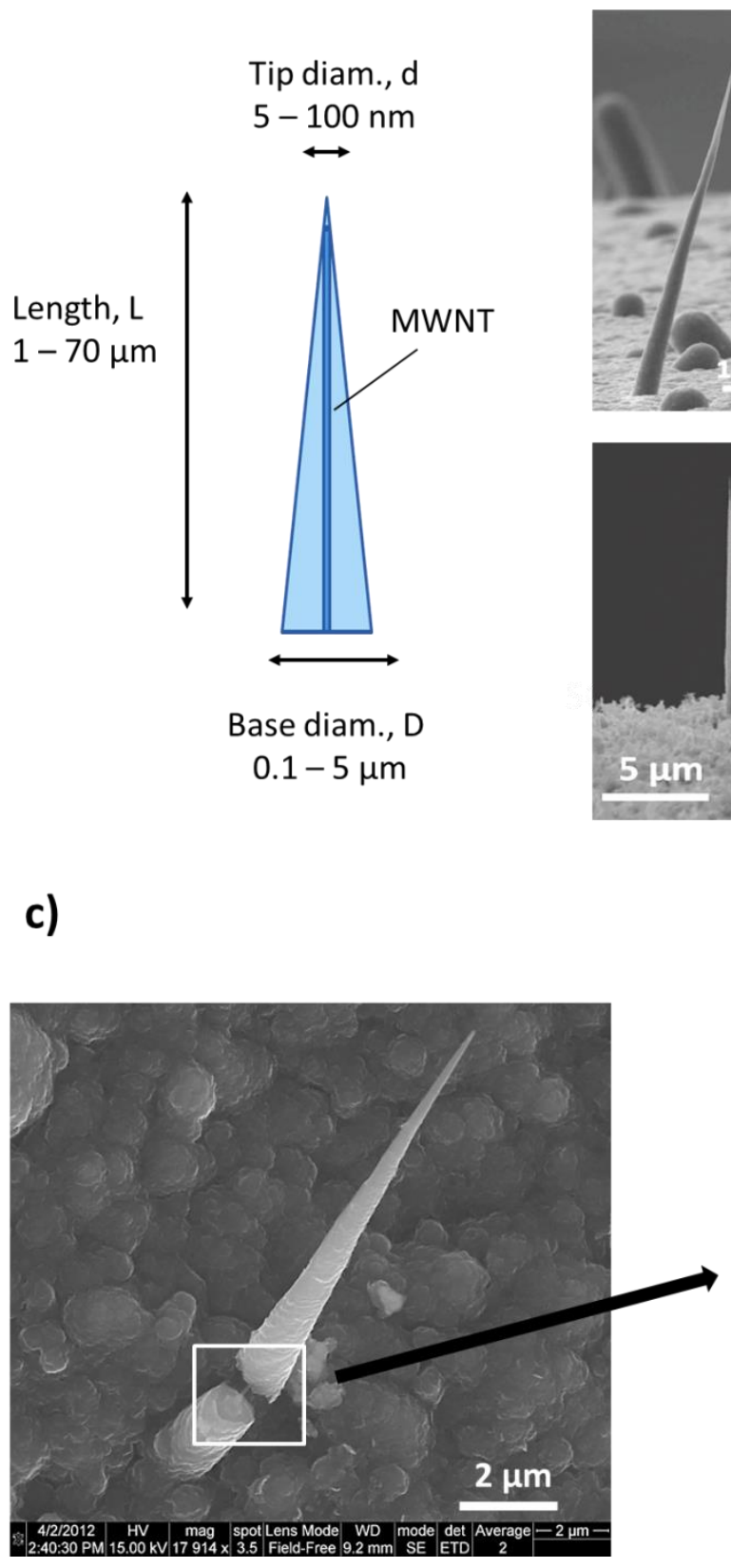

b) SEM image of the tip
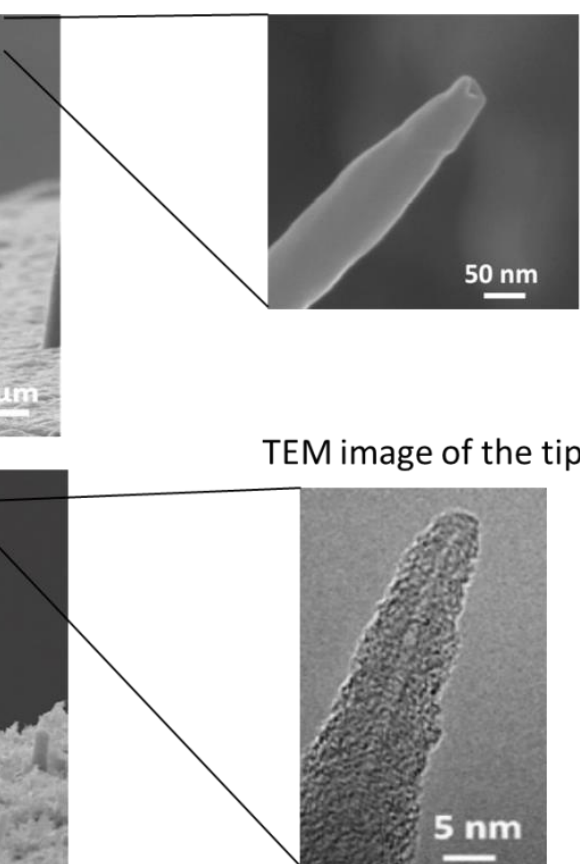
In this work we will concentrate on the samples with the array of the vertically aligned CCNTs grown on Pt wire and graphite foil. In this case the growth of CCNTs is uniform with respect to the coverage on the surface of the substrate and morphology (length and diameter of the tips and bases). In the case of Mo substrates the result of the growth was typically less uniform.

\subsubsection{Experimental procedure.}

Studied CCNT samples were grown by microwave plasma assisted chemical vapor) deposition (MWCVD) using AsTeX 5010 microwave plasma reactor (1.5 kW, $2.45 \mathrm{GHz}$ ) on two different substrates: (i) platinum wire (Alfa Aesar, $300 \mu \mathrm{m}$ diameter, 99.9\% (metals basis)) $[69,70]$ and (ii) graphite foil (Alfa Aesar, $130 \mu \mathrm{m}$ thick, $99.8 \%$ (metals basis)) with the sputtered thin layer $(30 \mathrm{~nm})$ of the Pt [70]. Total length of the Pt wire samples were $\sim 3-4 \mathrm{~cm}$ and average area of deposition was $0.02 \mathrm{~cm}^{2}(\sim 2 \mathrm{~mm}$ section of Pt wire on one end). For graphite foils, average area of deposition was $0.5 \mathrm{~cm}^{2}$ and total area of the sample $\sim 1 \mathrm{~cm}^{2}$. Samples grown on Pt wire are labeled 1, 2 and 3 while samples on graphite foil are labeled 4, 5 and 6 (Table 2). Substrates Pt wire and pure graphite foil (before sputtering) are usually cleaned in acetone sonication bath. Pt wires were placed vertically in graphite susceptor (Figure $4.2 \mathrm{~b}$ ) inside the vacuum chamber. Graphite foils after Pt sputtering were rolled around $1 \mathrm{~mm}$ graphite rod and then placed vertically in graphite susceptor as well (Figure $4.2 b$ ). 
a)

MWCVD chamber

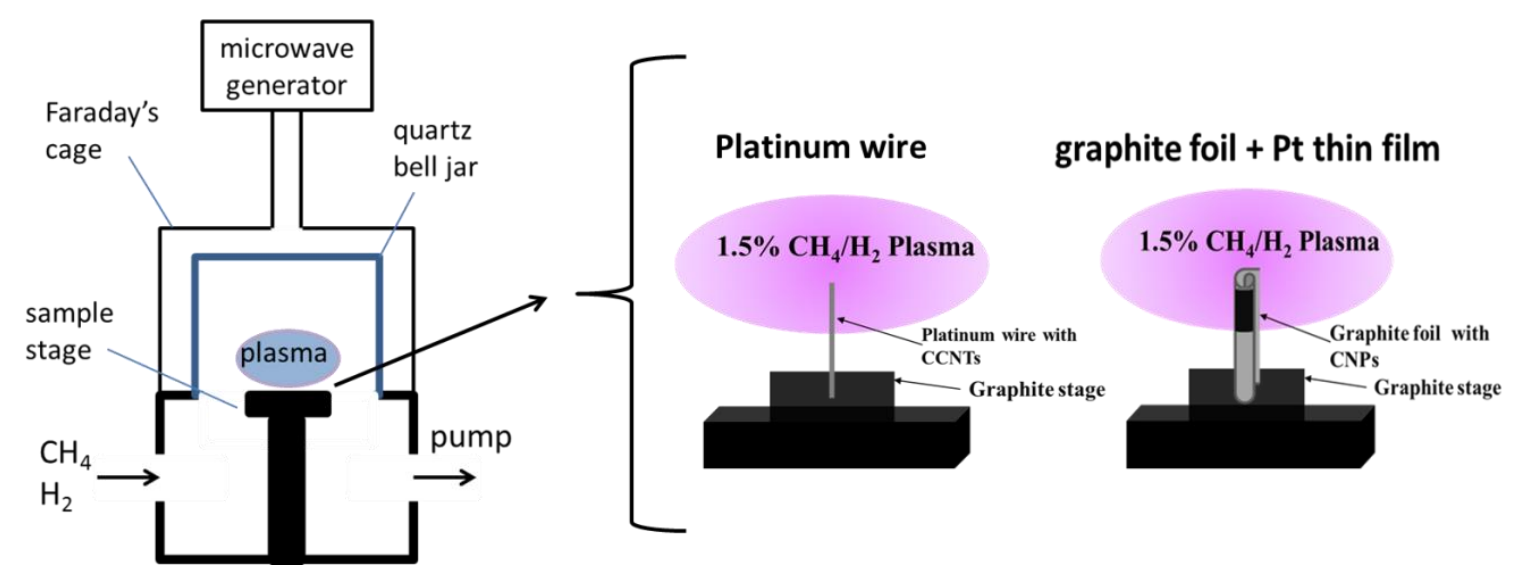

Figure 4.2 a) Schematic view of the vacuum chamber for MWCVD growth and b) arrangement of the substrates $\mathrm{Pt}$ wire and graphite foil on the susceptor inside the chamber.

More detailed experimental procedure of the growth and sample preparation has been described in detail elsewhere $[69,70]$. However, some changes during the growth process were introduced for this particular study for both samples grown on the Pt wire and graphite foil. The experimental procedure is still similar to that of previous work [70], except for the addition of a two-step process with a change in the gas phase composition to vary the structural characteristics of CCNTs. Step 1 consists of carbon deposition using $1.35 \%$ (Pt wire) or 2.5 vol. \% (graphite foil) methane in $200 \mathrm{sccm}$ of hydrogen followed by a deposition and etching with $1 \%$ (Pt wire) or 2 vol. \% (graphite foil) methane in $200 \mathrm{sccm}$ of hydrogen in step 2 . Time of the $1^{\text {st }}$ step is $15 \mathrm{~min}$ and of the 2 step 10 min. Pt wire samples were synthesized at pressures 25 torr and microwave power $950 \mathrm{~W}$; graphite foil samples at $30-50$ torr and microwave power $900-1000 \mathrm{~W}$. 
Final important detail is that during the growth both type of substrates were immersed vertically into plasma (Figure 4.2b).

\subsubsection{CCNT - Result of the growth.}

As a result of the MWCVD growth, samples were obtained with an array of randomly scattered Conical Carbon Nanotubes (CCNTs) on the substrates. Figure 1 shows the SEM images of the four CCNTs samples under investigation. There are visible variations in density, morphology, and aspect ratio. The insets show the enlarged view of the individual CCNT tip. The close-up view of sample 6 as shown in Fig. 4.3 (e) shows presence of "horn-like" structures with blunt tips along with CCNTs. The characteristics for the CCNT samples are summarized in Table 2.
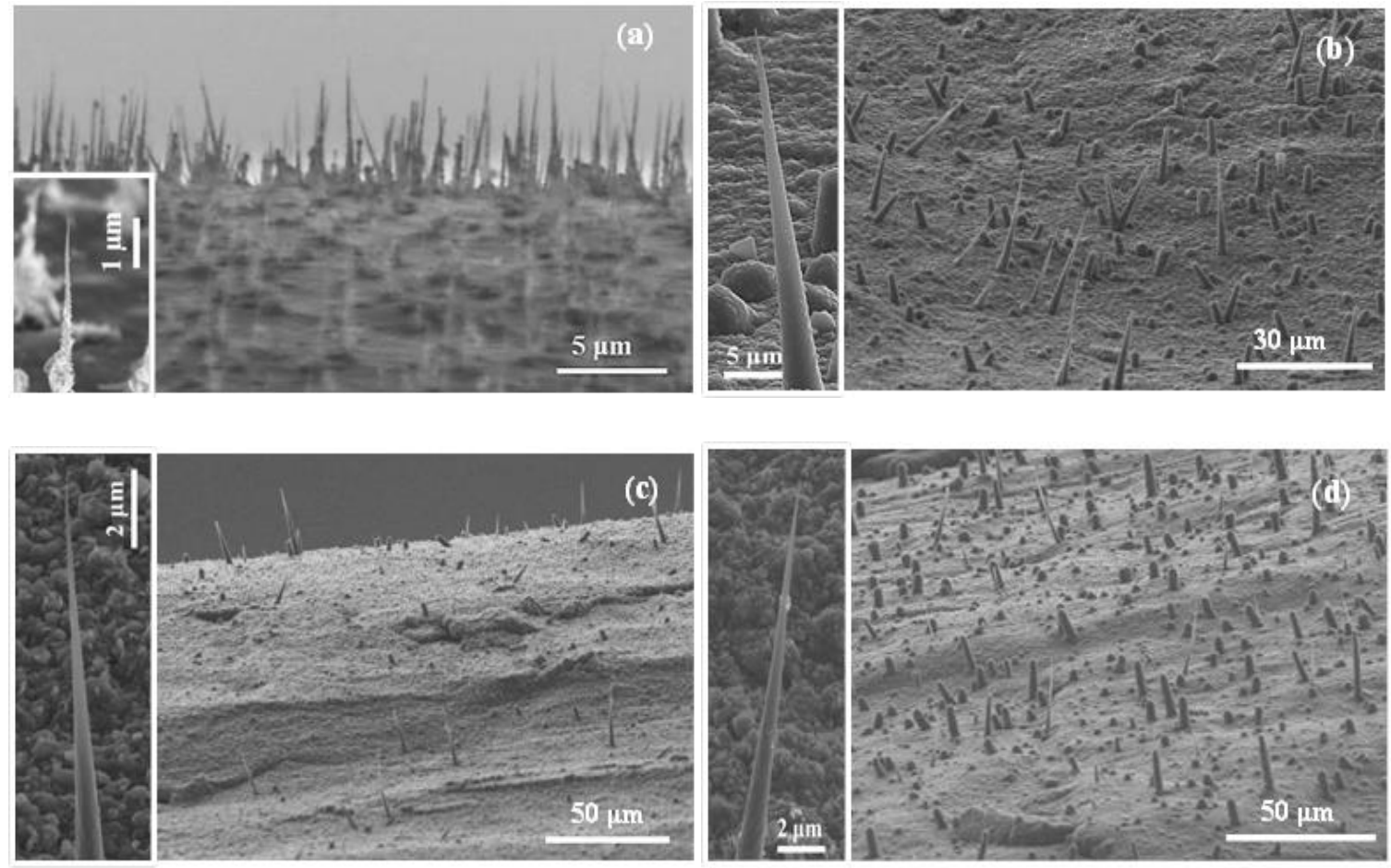


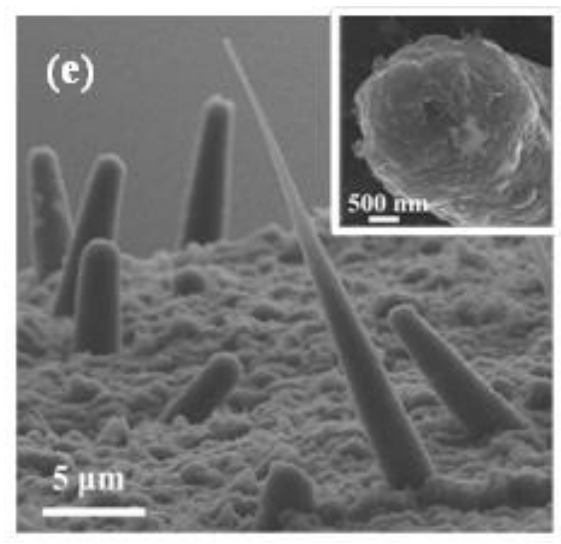

Figure 4.3 SEM images of array of CCNTs on Pt wire: (a) sample 1. On graphite foil: (b) sample 4, (c) sample 5 and (d,e) sample 6 with insets showing the enlarged view of the corresponding CCNT tip. The image (e) shows presence of microhorns structure along with CCNTs with inset showing top part of the microhorn. Images were obtained using NOVA Nano SEM 600. (Used with permission - Apendix A).

Table 4.1 Characteristics of the CCNTs grown on Pt wire and graphite foil.

(Used with permission - Apendix A).

\begin{tabular}{|c|c|c|c|c|c|}
\hline & CCNT sample & $\begin{array}{l}\text { Length }^{1}, \\
1[\mu \mathrm{m}]\end{array}$ & $\begin{array}{c}\text { Base } \\
\text { Diameter }^{1} \text {, } \\
\mathrm{D}[\mu \mathrm{m}]\end{array}$ & $\begin{array}{l}\text { Tip Diameter } \\
\text { d }[\mathrm{nm}]\end{array}$ & $\begin{array}{c}\text { Aspect ratio, } \\
\text { 1/D }\end{array}$ \\
\hline $\begin{array}{c}\mathrm{Pt} \\
\text { wire }\end{array}$ & $1-3$ & $5-10$ & $0.1-0.5$ & $10-20$ & $50-100$ \\
\hline \multirow{4}{*}{ 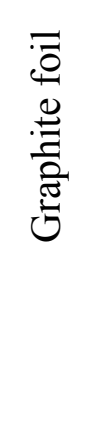 } & 4 & $15-30$ & $2-4$ & $30-50$ & $10-30$ \\
\hline & 5 & $2-15$ & $0.5-1$ & $50-100$ & $4-30$ \\
\hline & $\begin{array}{c}6 \\
(\mathrm{CCNT})\end{array}$ & $15-25$ & $1-2.5$ & $60-100$ & $15-25$ \\
\hline & (“microhorns") & $0.5-50$ & $3-4$ & $500-2000$ & $1.7-10$ \\
\hline
\end{tabular}

${ }^{1}$ Length and diameter of CCNT are averaged values obtained from characterization of several different areas of each sample. 
CCNTs grown on platinum wire are usually smaller in size with aspect ratio higher than in the case of graphite foil. The density of the growth of the CCNTs for Pt wire is on average significantly larger $\left(10^{7} / \mathrm{cm}^{2}\right)$ in comparison with graphite foil samples: sample (4) $-10^{4} / \mathrm{cm}^{2}$; sample (6) $-10^{2} / \mathrm{cm}^{2}$ for both CCNTs and microhorns, respectively. However, these density values are rough estimates, as it is considered as an array of randomly grown CCNTs.

\subsection{CCNT - Thermionic Emission and UPS measurements.}

\subsubsection{Thermionic emission - experimental.}

Thermionic emission and field emission measurements were performed on each sample in a vacuum chamber at a base pressure about $10^{-7}$ Torr. Two different arrangements of measurements were used for the CCNTs grown on (i) platinum wire and (ii) graphite foil (Figure 4.4).
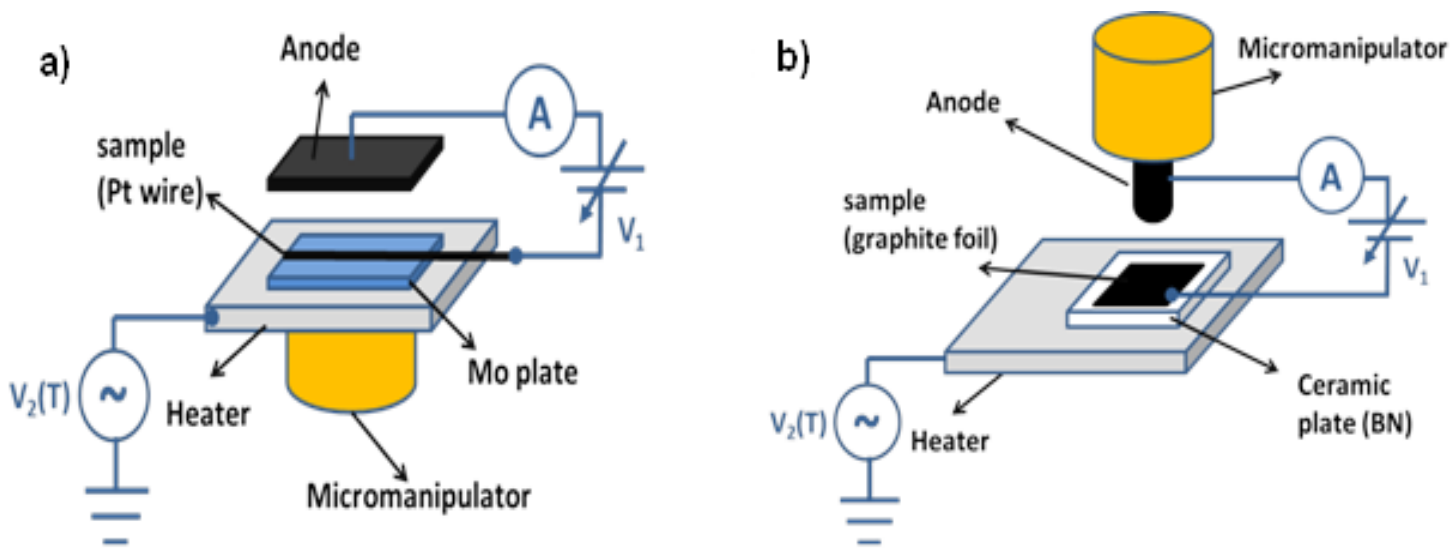

Figure 4.4 Field emission/thermionic emission measurement set-up for CCNTs grown on: (a) Pt wire; (b) graphite foil. (Used with permission - Apendix A). 
In the case of the platinum wire, the sample was placed in a V-groove of a molybdenum plate (Figure 2 (a)). A flat molybdenum anode was slowly moved toward the CCNTs by means of a micromanipulator. In the second case, conducting graphite foil with synthesized CCNTs was placed on a thin ceramic plate (Boron Nitride) supported on a Pyrolitic Boron Nitride (PBN) heater (Fig. 2 (b)). A conducting molybdenum wire was attached mechanically to the graphite foil so that wire and foil act as the cathode. A molybdenum anode was arranged over the sample and attached to the micromanipulator (Fig. 2 (b)). Zero distance $(\mathrm{d}=0)$ between cathode (sample) and anode was established by observing a sudden electrical short when the anode just touched the sample. Measurements were performed at a set distance (d) for different temperatures by sweeping the voltage $U$ from 0 to $500 \mathrm{~V}$ while recording the current $I$ using a picoammeter (Keithley 6487) equipped with a built-in variable voltage source. PBN heater was connected to a separate power supply. Temperatures were measured using an infrared pyrometer (Raytek MA2SCCF; Infrared; single color; Spectral response: 1.6 $\mu \mathrm{m})$.

After loading the samples into the chamber and reaching desired pressure, field emission measurements were performed. I-U characteristics of the CCNTs were studied at room temperature for varying separations between the cold cathode and the anode. This was done in order to define conditions for which field enhanced thermionic emission will be the dominating mechanism of the electron emission to the vacuum and to obtain information about field emission properties of our samples. Separation distances between anode and cathode for thermionic emission measurements were typically $1000-2000$ $\mu \mathrm{m}$. 


\subsubsection{UPS - Experimental.}

UPS measurements were performed using multi-chamber ultra-high vacuum (UHV) surface science facility (VG Scientific/ RHK Technology) comprising of a 150 mm radius CLAM 4 hemispherical analyzer. CCNT arrays on platinum wire were studied using He-I (21.23 eV) and He-II (40.81 eV) UV excitations. A stable bias was provided to avoid the instrumental cutoff in the lens system of the analyzer at low kinetic energy (KE) for all the UPS spectra measurements. The external bias and the spectra were shifted back to zero-bias position through data post-processing. The calibration of the UPS spectrometer was performed by measuring and validating the absolute position of the Fermi level of a standard gold sample.

\subsubsection{Thermionic Emission and UPS of the CCNT - Results and discussion.}

The thermionic Current-Voltage characteristics measured at various temperatures are shown in Figure $4.5 \mathrm{a}$, b. These are results obtained from the CCNT grown on the Pt wire, sample 1. It is evident that I-V curves have a characteristic shape for FETE (Schotky curve). Similar to experimental I-V curves for MWNT and MWNT yarn or sheets discussed in chapter 3.1.2. For the I-V curves one can distinguish a low voltage region with a dramatic increase of the emission current with the voltage (retarding field regime) followed by a saturation-like region at higher voltages (accelerating field regime) $[1,9,32,70,73,74]$. Thus we can use Schottky equations 2.31 and 2.34 from the chapter 2. As noted before, in practice, instead of current density $J$ and electric field $E$ we consider current 

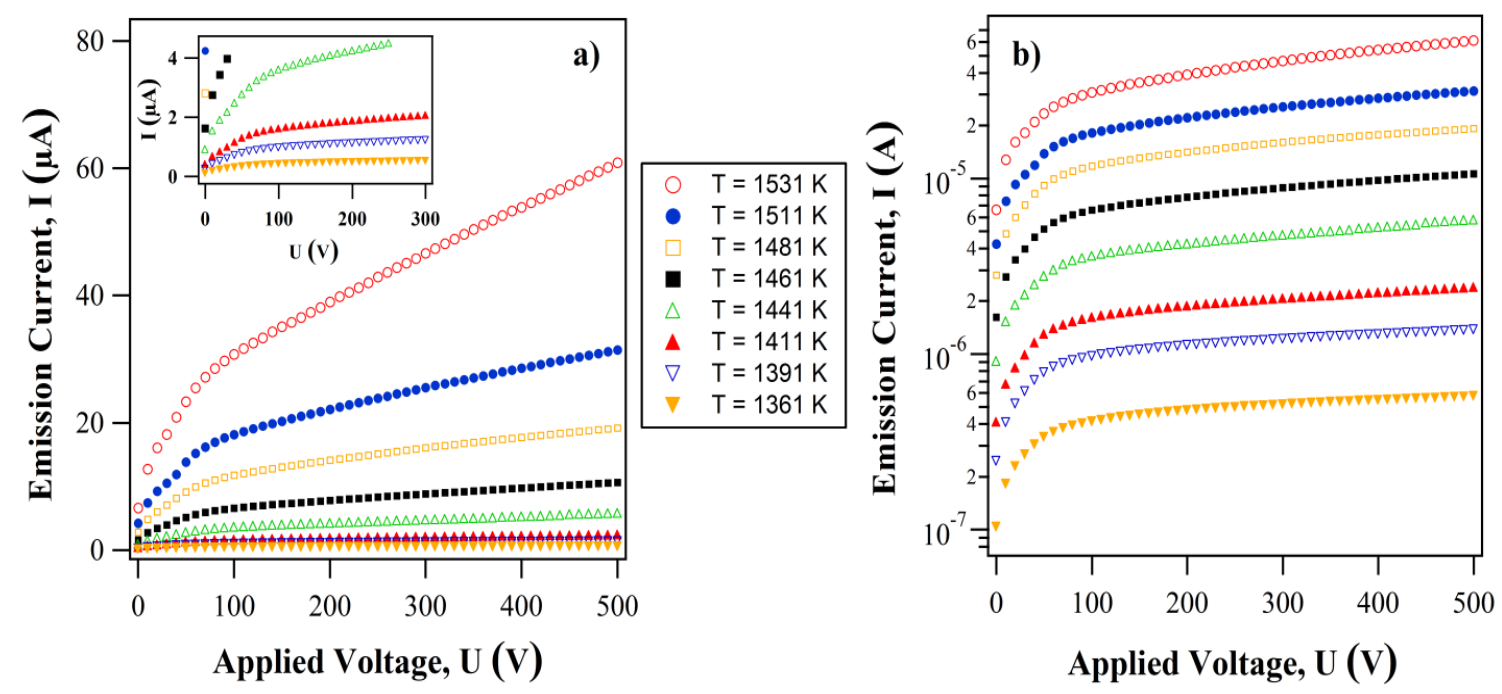

Figure 4.5 (a) Emission current versus applied voltage at different temperatures for CCNT grown on platinum wire, sample 1; (the inset shows magnified $I-U$ curves at lower temperatures); (b) $I-U$ curve for various temperatures same as in (a) but the current $I$ (for clarity) in logarithmic scale. (Used with permission - Apendix A).

$I$ dependence on temperature $T$ and applied voltage $U$. Zero field emission current, $I_{0}$, is determined from thermionic $I-V$ (Figure 4.6 a) characteristics and $\operatorname{Ln}(I)$ vs $\sqrt{U}$ curves (Figure 4 a) for each temperature [9,32]. By extrapolating linear saturation part of $\operatorname{Ln}(I)$ vs $\sqrt{U}$ curves one can obtain the value of zero field current $I_{0}$ modifying equation (2.31),

$$
L n J=L n J_{0}+\frac{C}{k T} \sqrt{E}
$$

Once the values of $I_{0}$ are known, by plotting $\operatorname{Ln}\left(\frac{I_{0}}{T^{2}}\right)$ vs $\frac{1}{T}$ (Figure 4(b)) one can find the work function from the slope. The temperature dependence of zero field current, $J_{0}$ is plotted in Figure 4 (c), which follows Richardson-Dushman model. Since current density, $J$ is defined as current per unit area, $J=I / S$ and use of $\operatorname{Ln}(J)=\operatorname{Ln}\left(\frac{I}{S}\right)$ in eq. (3) implies 
that intercept $\operatorname{Ln}\left(I_{0}\right)$ constitutes the emission area $S$ [32]. However, the slope (work function) of the linear fit, $\operatorname{Ln}\left(\frac{I_{0}}{T^{2}}\right)$ vs $\frac{1}{T}$ does not depend on the size of the area $S$.
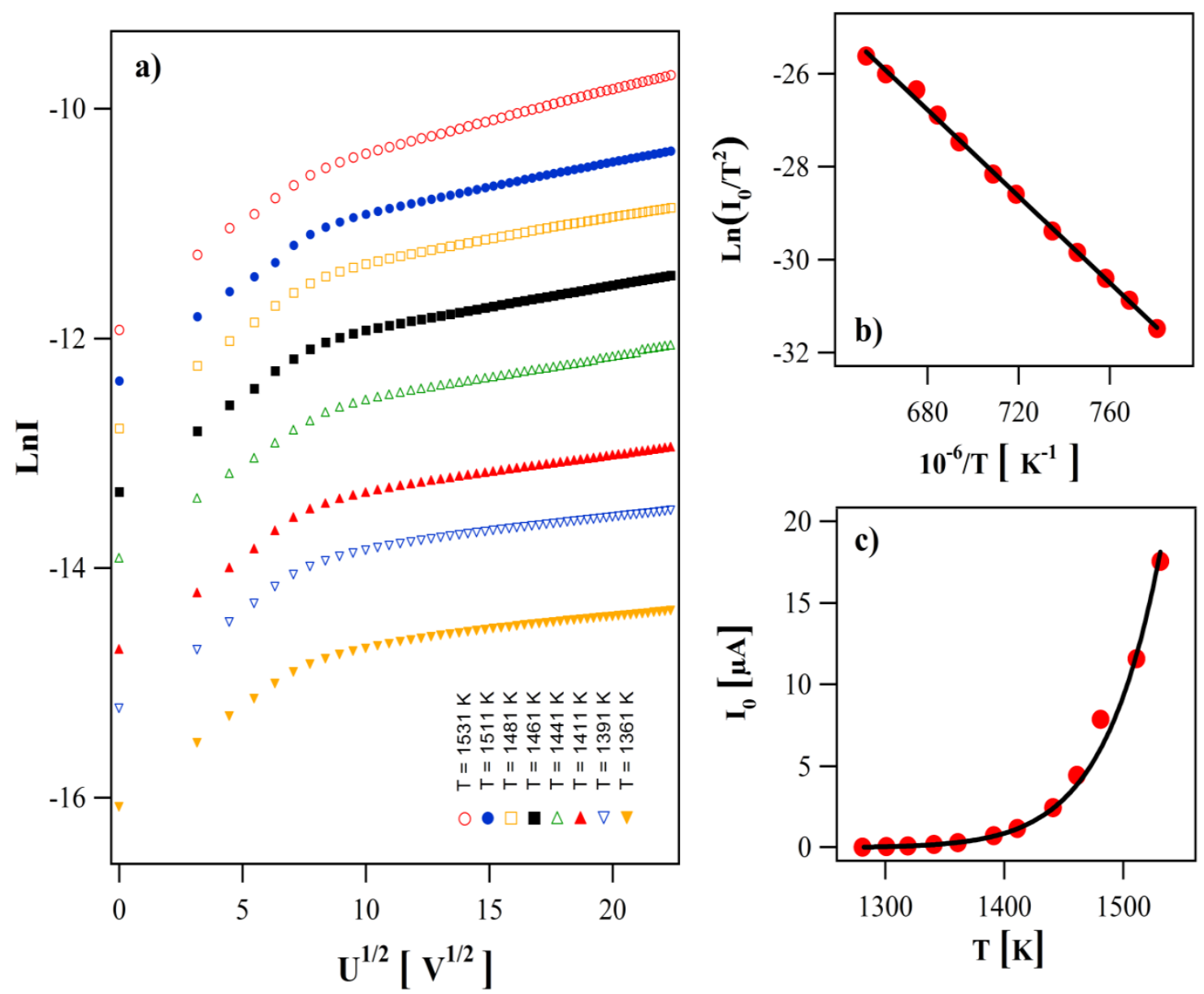

Figure 4.6 (a) $\operatorname{Ln}(I)$ vs $U^{1 / 2}$ curves showing low voltage retarding field regime followed by linear higher voltages accelerating field regime for sample 1 ; (b) $\operatorname{Ln}\left(I_{0} / T^{2}\right)$ versus (1/T) plot; (c) Zero field current $I_{0}$ vs $T$. (Used with permission - Apendix A).

The value of work function for CCNTs grown on platinum wire is calculated to be $\sim 4.1 \mathrm{eV}$. This is significantly lower than values reported for carbon nanotubes, which are usually on average $\sim 4.6-5 \mathrm{eV}$ (Table 4.1).

For the samples with CCNT's grown on graphite foil the value of work function was obtained using the same method. 

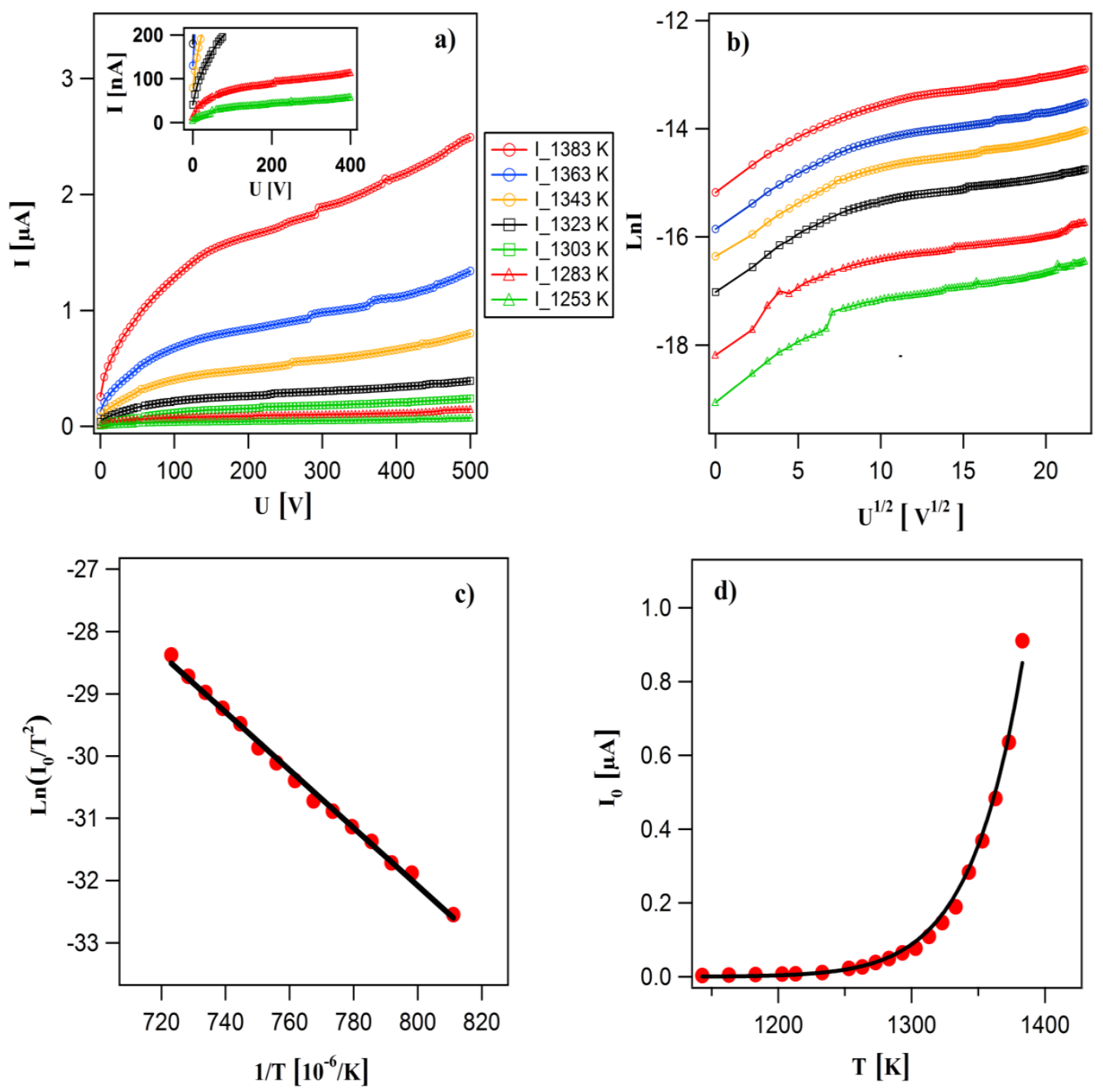

Figure 4.7 Sample 4 grown on graphite foil : (a) $I$ - $U$ characteristic; (b) $\operatorname{Ln}(I)$ vs $U^{1 / 2}$; (c) Ln $\left(I_{0} / T^{2}\right)$ versus $(1 / T)$ plot with best linear fit; (d) Richardson - Dushman curve, $I_{0}$ vs T. (Used with permission - Apendix A).

Fig. 5(a) shows the thermionic current, I vs. the cathode-anode voltage, $U$ for various temperatures. Fig. 5(b) shows $I$ vs $U^{1 / 2}$ plot for each temperature which will be used to extract $I_{0}$ values as described earlier. Fig. 5(c) shows the $\operatorname{Ln}\left(I_{0} / T^{2}\right)$ versus $(1 / T)$ plot for each temperature with the best linear fit whose slope is used to calculate the work function, $\Phi$. The temperature dependence of the zero field current, $I_{0}$ satisfies the Richard-Dushman model as shown in Figure 5(d). The analysis yields a value of $4.1 \mathrm{eV}$ 
for $\Phi$ for sample 4 and for samples 5 and $6-4.3 \mathrm{eV}$ and $4.7 \mathrm{eV}$, respectively. Thermionic emission results for all samples are shown in table 4.2 and Richardson-Dushman plots for all 3 CCNT samples grown on the graphite foil are shown in the Figure 4.8.
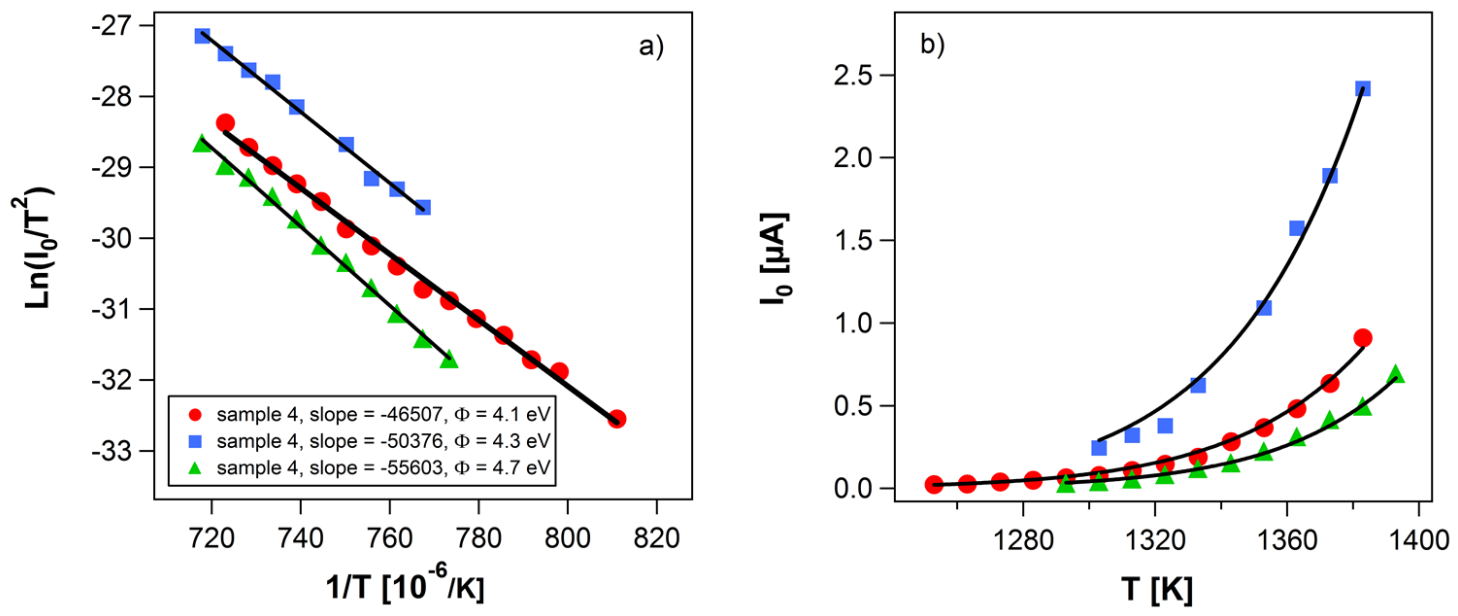

Figure 4.8 Richardson-Dushman plots for all three samples grown on the graphite foil (samples 4-6): a) linear $\operatorname{Ln}\left(I_{0} / T^{2}\right)$ versus $(1 / T)$ plots; b) the temperature dependence of the zero field current, $I_{0}$.

It has been found that this value of 4.1- $4.3 \mathrm{eV}$ for samples 1-5 (except sample 6) is smaller than the value claimed for most of the multi-wall carbon nanotubes. The onset of thermionic emission occurs at a temperature as low as $900{ }^{0} \mathrm{C}$ which is lower than the reported temperatures in thermionic emission of the sidewalls of MWNT and in some case tips [32]. Though comparable to the value $\phi$ from vertically aligned MWNT [35]. These properties could possibly be attributed to the local electric field enhancement. It was shown that CCNTs have high aspect ratio and excellent field enhancement factors in addition to sparse distribution, reducing the screening effect [69]. The previously described structural differences of sample 6 in comparison with other CCNT samples (presence of microhorns) could be the reason for the higher observed value for work function. 
In order to determine the work function by Ultraviolet Photoemission Spectroscopy (UPS), CCNTs grown only on platinum wires (significantly higher work function $5-6 \mathrm{eV}$ ) were studied. Figure 6 shows the low kinetic energy part of the He-I emission spectra of CCNT arrays on a platinum wire. The work function was then determined from the intersection of low-KE cut-off tail with the background level. Value of $4.5 \mathrm{eV}$ was obtained for sample with CCNT arrays on platinum wires.

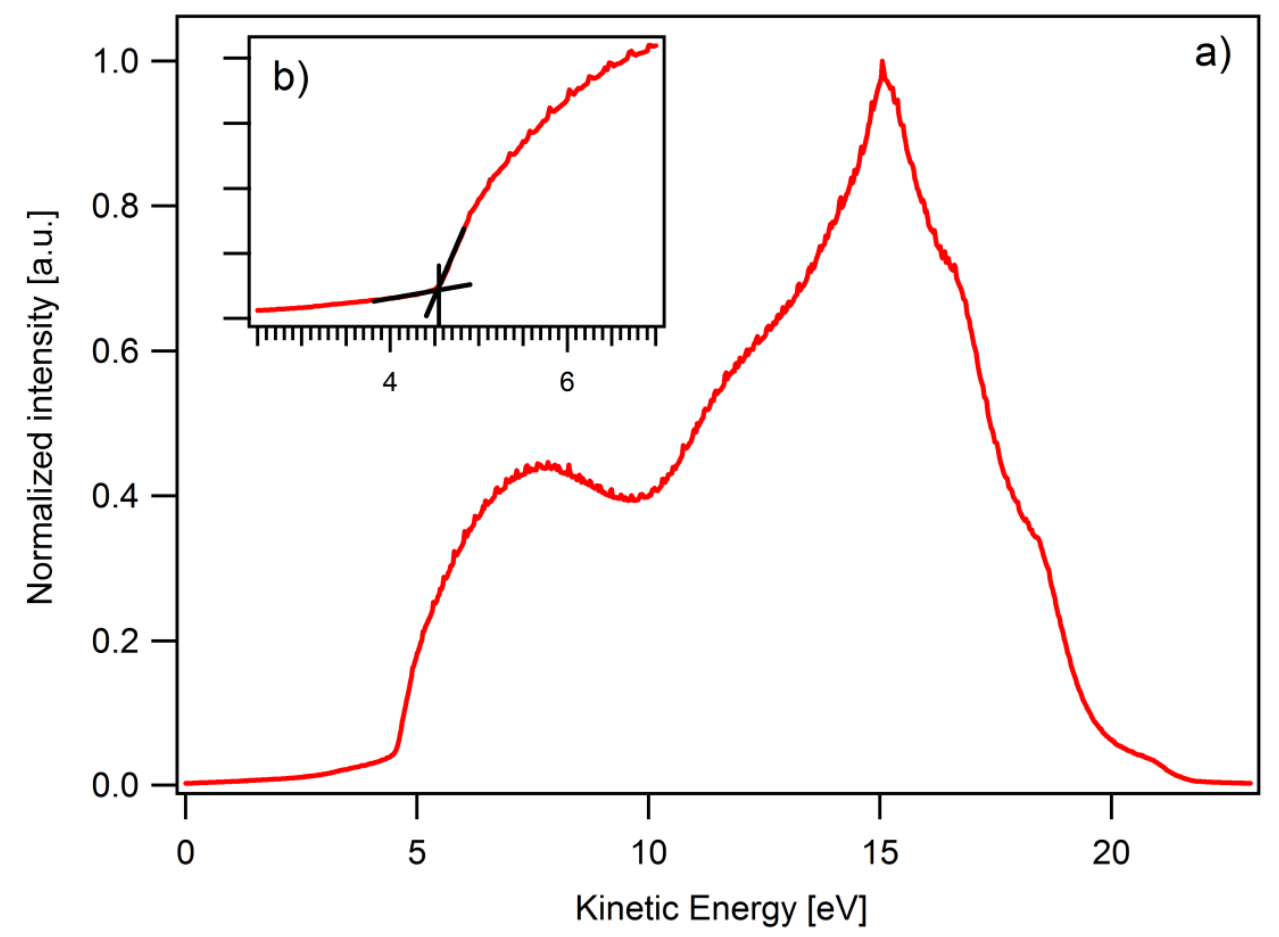

Figure6 4.9 a) Low kinetic-energy He - I (21.23 eV) spectra of CCNT arrays on platinum wire (sample 3 ) with the inset b) showing low kinetic cut-off energy positions indicative of work function. (Used with permission - Apendix A).

As mentioned, room temperature field emission properties of all the samples were studied for different anode-cathode separation distances $\boldsymbol{d}$ (Figure 4.9). These measurements could provide additional important information that can help understand the wide range of $\phi$ values for the six samples with different morphologies on different substrates. 
Figure 4.10 shows a typical Fowler-Nordheim curves for different separation distances. The value of $\boldsymbol{\beta}$ is determined using simplified Fowler-Nordheim equation 2.22, 2.27 as described in the chapter 2.3: $J=\frac{a E_{b c}^{2}}{\Phi} e^{-\frac{b \Phi^{\frac{3}{2}}}{E_{b c}}}[24,28]$; where, $a=1.54 \cdot 10^{-6}$ $(\mathrm{eV}) \mathrm{A} / \mathrm{V}^{2} ; b=6.83 \cdot 10^{9} \mathrm{~V} /(\mathrm{eV})^{3 / 2} \mathrm{~m}$. And $E_{l o c}=\boldsymbol{\beta} \cdot E_{a p p} ; E_{l o c}-$ local $($ effective) electric field; $E_{a p p}$ - applied electric field, $E_{a p p}=U / d$. Value of $\phi$ is obtained from thermionic emission measurements.

Table 4.2 Summary of the work function values of each CCNT sample grown on graphite foil and on platinum substrate. (Used with permission - Apendix A).

\begin{tabular}{|c|c|c|c|c|c|}
\hline & $\begin{array}{c}\text { Sample } \\
\quad \#\end{array}$ & $\begin{array}{l}\text { Work function, } \\
\Phi(\mathrm{eV})\end{array}$ & $\begin{array}{l}\mathrm{d} \beta / \mathrm{dx} \\
\text { (slope) }\end{array}$ & $\begin{array}{c}\beta \\
\left(\begin{array}{c}\text { value at } 120 \\
\mu \mathrm{m})\end{array}\right.\end{array}$ & $\begin{array}{c}\text { Aspect } \\
\text { ratio, } \\
\mathrm{d} / 1\end{array}$ \\
\hline \multirow{2}{*}{$\begin{array}{l}\text { Platinum } \\
\text { wire }\end{array}$} & 1,2 & 4.1 & 18.09 & \multirow{2}{*}{$\sim 2000$} & \multirow{2}{*}{$\sim 100$} \\
\hline & 3 & $\begin{array}{c}4.2 \text { (TE) } \\
4.5 \text { (UPS) }\end{array}$ & & & \\
\hline \multirow{3}{*}{$\begin{array}{l}\text { graphite } \\
\text { foil }\end{array}$} & 4 & 4.1 & 15.71 & 1536 & $10-30$ \\
\hline & 5 & 4.3 & 8.52 & 1040 & $4-30$ \\
\hline & 6 & 4.7 & 7.39 & 1134 & $\begin{array}{c}15-30 \\
(1.7-10)\end{array}$ \\
\hline
\end{tabular}


a)

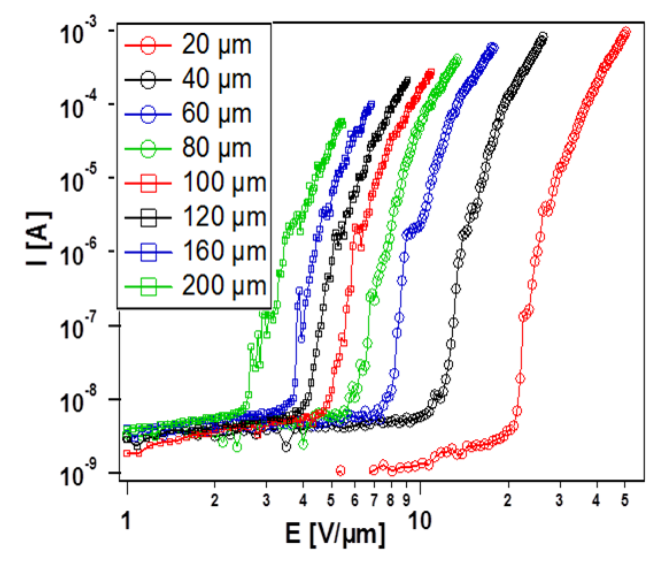

b)

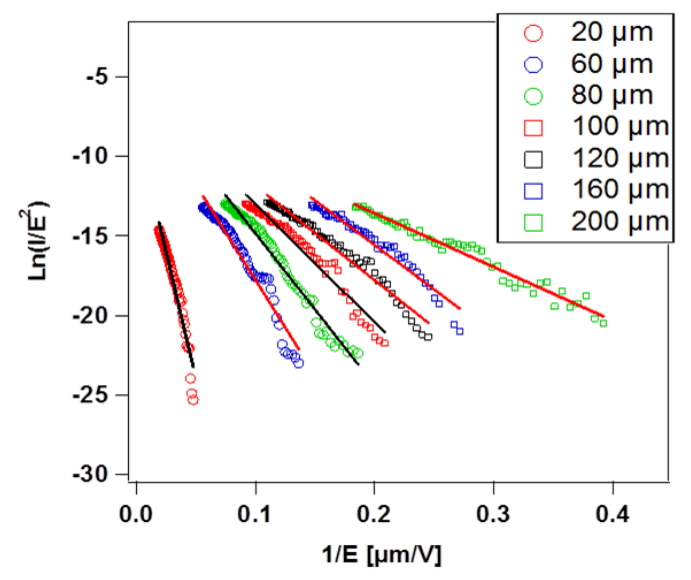

Figure 4.10 Field emission measurements of the sample 4: a) current I dependence on electric field E; b) corresponding Fowler-Nordheim curves.

It is known that $\boldsymbol{\beta}$ depends on the inter-electrode distance (d) and other factors such as tip radius, aspect ratio [71,76] and field screening effect [71,77]. In the case of CCNTs grown on different substrates, the discrepancy can be attributed to the structural characteristics of these conical morphologies with open edges on the outer surface acting as emission sites. From Figure 4.11 it is clearly seen that field enhancement is increasing with distance at a higher rate for samples with lower work function.

At a separation distance $\sim 120 \mu \mathrm{m}, \boldsymbol{\beta}$ values for samples 1 and 4 are above 1500, while for samples 5, 6 it remains less than 1000. It should be noted that samples 1 and 4 have larger aspect ratio and higher density of the CCNTs compared to samples 5 and 6 (Tables 4.1, 4.2). 


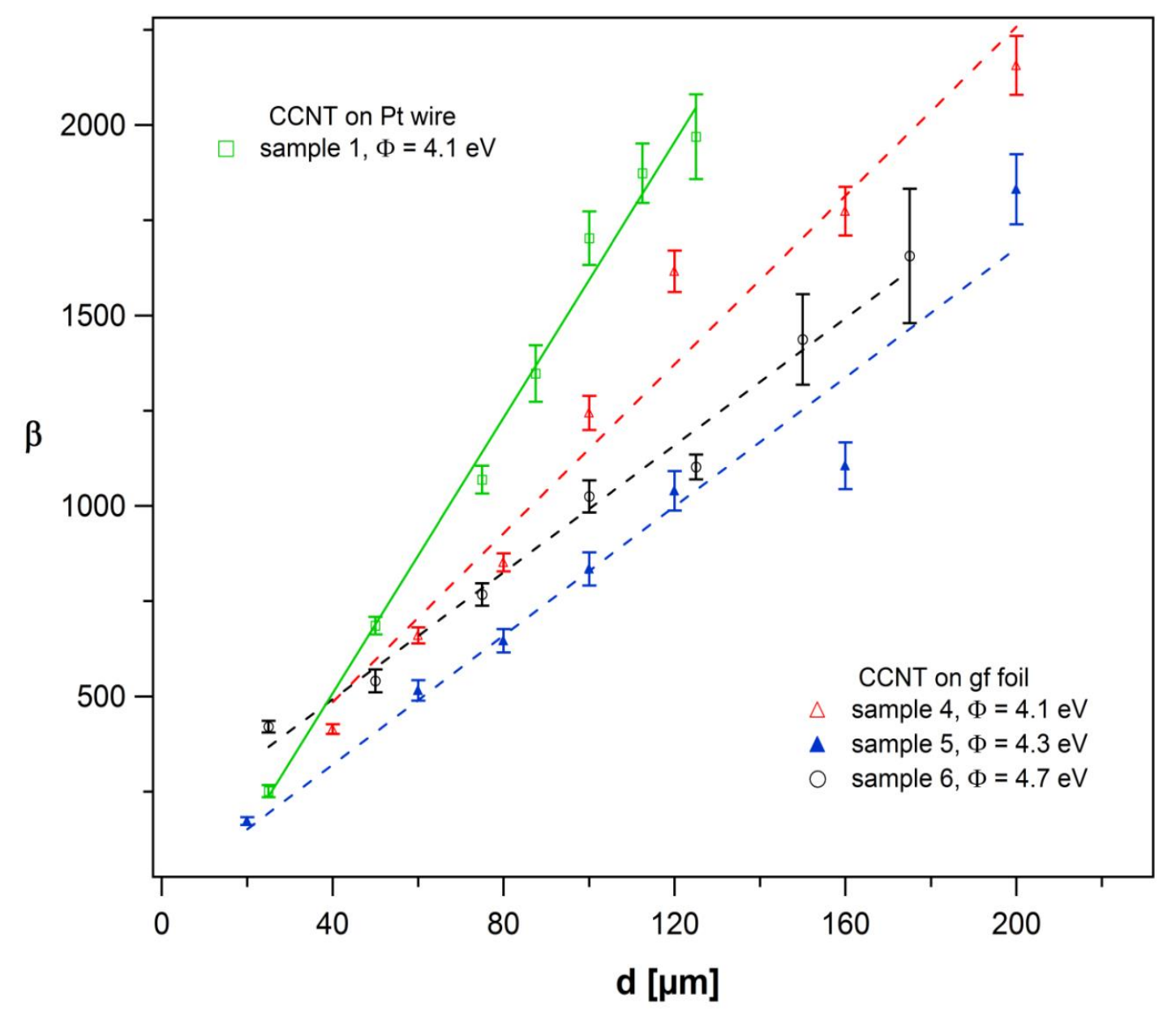

Figure 4.11 The field enhancement factor, $\beta$ vs. cathode-anode separation $\boldsymbol{d}$ for the four CCNT representative samples grown on graphite foils. (Used with permission - Apendix A).

The reduction of work function of CCNTs could be related to geometric electric field enhancement $\boldsymbol{\beta}$ which induces local large fields at the tip of the CCNT. The presence of such local fields at the tip of CCNT could lead to the field penetration effect, which causes a reduction of the effective work function [24]. It was shown in a theoretical study that for SWNT (with open tip), field penetration can reduce the value of effective work function even by $\sim 1.8 \mathrm{eV}$ [24]. It has been reported that the magnitude of the electric field to which SWNT was exposed is $\sim 0.33 \mathrm{~V} / \AA$. A. Mayer et al have shown 
that the field penetration effects could be more pronounced for the MWNT, especially the one with open convex tip [24].

It was illustrated at th beginning of this chapter that specific structural feature of CCNTs studied in this work is that they consist of an MWNT at the core along the axis of the CCNT. Concentric graphene layers are deposited around MWNT during the subsequent synthesis [69]. The number of graphene layers is gradually reduced towards the tip, where MWNT is exposed, which allows us to apply a field penetration model for CCNTs.

For a cathode-anode separation of $\sim 2000 \mu \mathrm{m}$, the maximum applied electric field at the tip of the cathode, $\mathrm{E}_{\text {applied,max }}=0.25 \mathrm{~V} / \mu \mathrm{m}$ during the thermionic emission measurements. Assuming a value of $\boldsymbol{\beta}$ of 2000 extracted from the field emission measurements for the largest cathode-anode separation of $200 \mu \mathrm{m}$ for the sample 1 , the local electric field for sample 1 will be

$E_{l o c}=\beta \cdot E_{\text {applied, } \max }=2000 \cdot 0.25 \mathrm{~V} / \mu \mathrm{m}=500 \mathrm{~V} / \mu \mathrm{m}$

Electric field of such magnitude according to the proposed field penetration model for SWNT [24] will reduce the potential barrier by $\sim 0.4 \mathrm{eV}$. While it is a rough estimation and one must consider the fact that the CCNTs have an exposed MWNT at the tip, we argue that our results of thermionic emission are consistent with the model in which field penetration is the main contributor for lowering the potential barrier and reduction of the value of the effective work function. It is also important to note that low densities of the CCNTs make the screening effect negligible [69].

The relationship between the values of effective work function and field enhancement factors for other samples (Table 4.2) seems to be in agreement with this 
model with the exception of sample 6. Even though this sample has a higher value of work function $(\sim 4.7 \mathrm{eV})$, the $\boldsymbol{\beta}$ value is higher than that for sample 5. It might be due to the presence of horn-like structures (microhorns) on the graphite foil in addition to CCNTs. Microhorns do not have exposed MWNTs and the aspect ratio is smaller compared to CCNTs (Table 4.1, Figure 4.3 (e)). However, the density of microhorns is comparable to that of CCNTs. As a result, microhorns could contribute to the increase of the value of work function for sample 6. It is important to note that the influence of the field enhancement on thermionic emission properties of CCNTs in relation to the screening and field penetration effects is a more complex problem; it can depend on various parameters such as density, aspect ratio, magnitude of applied electric field, and temperature.

The difference between the values of the work function obtained by UPS (Figure 6) and thermionic emission for sample 1 is also consistent with the field penetration model. As stated before, the presence of an electric field can cause a reduction of the potential barrier of CCNTs. This is obviously not the case for UPS. In addition, though UV photo-electro spectroscopy analysis was done under UHV conditions, the samples previously exposed to air were not annealed. Absorption of the gases at the tip of the CCNTs could have affected the UPS measurements.

Finally, we would like to comment on the field penetration effect in relation to previously published works presenting values of work function of carbon nanotubes obtained by similar methods (field enhanced thermionic emission) [33-37]. Influence of the field penetration effect has not been discussed in any of these articles.

As we noted before the earliest thermionic emission results from random films of SWNT and MWNT (purified nanotube paper) showed unusually low values of work 
function for CNT: $1.2 \mathrm{eV}$ [38] and $3.46 \mathrm{eV}$ [39]. These values are not in agreement with theoretical predictions or experimental results obtained by other methods [20-22]. The authors do not provide information about field emission measurements or field enhancement factors, nor many details about experimental procedure and number of tested samples. Hence it is hard to comment on these results in the context of the field penetration effect.

Thermionic emission measurements from MWNT yarn [32] and bundles of SWNT/DWNT/MWNT [33] produced results in agreement with theoretical calculations and other experimental methods as we discussed in chapter 3. The authors performed field emission measurements from the yarn and concluded that field emission comes mostly from carbon nanotube tips protruding out of the yarn (values of field enhancement $\boldsymbol{\beta}$ are not presented) and most of the thermionic emission comes from the CNT sidewalls. Primary factors for neglecting thermionic emission from the tips are small density of the exposed CNT tips in the yarn, small area of the tips $(10 \mathrm{~nm})$ and long length of the MWNT (several hundreds of microns). Based on these facts and presented work function results, it is hard to conclude whether field penetration effect should be considered in this case.

The same reasoning concerning field penetration effect could be applied to results of thermionic emission from the SWNT/DWNT/MWNT bundles [33]. It was reported that for the MWNT bundle with some tips appearing in the middle, the measured work function is smaller than without tips. Zhou et al. indicates that the work function of the tips is smaller than for sidewalls due to different electronic structure and image potential [20]. The authors do not present field emission data for CNT bundles and don not discuss 
field enhancement on the tips of the carbon nanotubes. Again, it is hard to conclude whether field penetration effect should be considered in these measurements.

The last cited article on thermionic emission measurements presents results from a vertically aligned multiwall carbon nanotube array [37]. The obtained value of work function $4.2 \mathrm{eV}$ is lower than the predicted value for CNT tip: $\sim 4.4 \mathrm{eV}$ [20]. The authors performed field emission measurements. The calculated value of field enhancement factor is comparable to ours $-\beta=1404$ (no information is provided about separation distance). No reasons for smaller work function for pristine MWNT are provided. We speculate that the work function could be reduced because of the field penetration effect.

Ultimately, it would be beneficial to estimate the values of thermionic emission current densities of CCNTs. First the surface of the entire sample was considered. Though the average effective area (area of deposition of CCNT) of Pt wire is smaller $\left(0.02 \mathrm{~cm}^{2}\right)$ than that of graphite foil $\left(0.5 \mathrm{~cm}^{2}\right)$, the density for CCNTs on platinum wire is much higher and an estimated number of CCNTs would still be larger than in the case of graphite foil.

Table 4.3 Estimated values of current densities for the whole sample and for individual CCNT. (Used with permission - Apendix A)

\begin{tabular}{|c|c|c|c|c|c|c|c|c|}
\hline & \multirow[b]{2}{*}{$\begin{array}{l}\text { CCNT } \\
\text { sample }\end{array}$} & \multirow[b]{2}{*}{$\begin{array}{c}\mathrm{T}, \\
{[\mathrm{K}]}\end{array}$} & \multirow[b]{2}{*}{$\begin{array}{c}I_{0} \\
{[\mu \mathrm{A}]}\end{array}$} & \multirow[b]{2}{*}{$\begin{array}{r}I_{F E T E}^{2} \\
{[\mu \mathrm{A}]}\end{array}$} & \multicolumn{2}{|c|}{ whole sample $^{1}$} & \multicolumn{2}{|c|}{ per CCNT } \\
\hline & & & & & $\begin{array}{c}J_{0} \\
{\left[\mu \mathrm{A} / \mathrm{cm}^{2}\right]}\end{array}$ & $\begin{array}{c}J_{F E T E}{ }^{3} \\
{\left[\mu \mathrm{A} / \mathrm{cm}^{2}\right]}\end{array}$ & $\begin{array}{c}J_{0, i}{ }^{4}{ }^{2} \\
{\left[\mathrm{nA} / \mathrm{cm}^{2}\right]}\end{array}$ & $\begin{array}{c}J_{F E T E, i}{ }^{5} \\
{\left[\mathrm{nA} / \mathrm{cm}^{2}\right]}\end{array}$ \\
\hline $\begin{array}{c}\mathrm{Pt} \\
\text { wire }\end{array}$ & 1 & $\begin{array}{l}1381 \\
1531\end{array}$ & $\begin{array}{l}0.74 \\
17.5\end{array}$ & $\begin{array}{c}1.37 \\
60\end{array}$ & $\begin{array}{c}74 \\
1750\end{array}$ & $\begin{array}{c}137 \\
6000\end{array}$ & $\begin{array}{c}0.74 \\
17.50\end{array}$ & $\begin{array}{c}1.37 \\
60\end{array}$ \\
\hline $\begin{array}{l}\text { Graphite } \\
\text { foil }\end{array}$ & 4 & 1393 & 0.91 & 2.5 & 1.82 & 5 & 0.18 & 0.5 \\
\hline
\end{tabular}


${ }^{1}$ Emission is considered from entire deposition area of the sample.

${ }^{2} \mathrm{I}_{\text {FETE, } i}$ - field enhanced thermionic emission (FETE) current value for applied voltage $U=500 \mathrm{~V}$;

${ }^{3} J_{\text {FETE }}$ - current density for the entire sample (or array of CCNTs) for $U=500 \mathrm{~V}\left(E_{a p p}=0.25 \mathrm{~V} / \mu \mathrm{m}\right)$.

${ }^{4} J_{0, i}$ - zero field current density per individual CCNT.

${ }^{5} J_{F E T E, i}$ - current density per individual CCNT for $U=500 \mathrm{~V}\left(E_{a p p}=0.25 \mathrm{~V} / \mu \mathrm{m}\right)$.

Also for Pt wire emission area we estimated $0.01 \mathrm{~cm}^{2}$ as only one semicircular arc of the wire is exposed to the anode. Using this value of area and measured currents, we estimated values of the zero field current density $J_{0}$ and field enhanced current density $J_{\mathrm{FETE}}$ which are presented in the Table 4.3.

The values of current densities from Table 4.3 were calculated using as the effective emission area, the area of the whole sample (CCNTs deposition area). Using this value of area and measured currents, we estimated values of the zero field current density $J_{0}$ and field enhanced current density $J_{\mathrm{FETE}}$ which are presented in the Table 4.3 For the whole deposition area of CCNTs array of sample 1, values of current densities are significantly larger than for sample 4 . Considering the current density per individual CCNT, the values for sample 1 are only slightly larger than for sample 4.

The value of FETE current density of the entire sample was reported only for vertically aligned MWNT array and it is comparable with ours [6]. At temperature $\mathrm{T}=$ $1386 \mathrm{~K}$ and applied electric field $E_{a p p}=0.25 \mathrm{~V} / \mu \mathrm{m}$, current density is $J_{F E T E} \sim 50 \mu \mathrm{A} / \mathrm{cm}^{2}$. None of the referred articles on thermionic emission from carbon nanotubes (with similar method of measurement) reported the values of current densities per individual carbon nanotube. The reported maximum value of thermionic emission current density from individual MWNT sidewalls was estimated to be $-2 \mathrm{~A} / \mathrm{cm}^{2}$ at temperature $\sim 2900 \mathrm{~K}$ [31]. This value was derived using a different experimental method - SEM in-situ 
measurements from MWNT sidewalls - with the assumption that the value of carbon nanotube work function was about $4.95 \mathrm{eV}$.

\subsubsection{Control measurements and conductivity of the CCNT.}

We performed the control experiment with bare graphite foil and bare platinum wire at high temperatures and did not observe any significant emission at the given anode-cathode separation $(1 \mathrm{~mm})$. The data for bare graphite foil are presented in Figure 8. Measurements were performed on a $1 \mathrm{~cm}^{2}$ sample of pristine graphite foil. Separation distance was maintained at $\sim 1 \mathrm{~mm}$. In Figure 4.12 (a) are compared $I-V$ curves for CCNT sample 1 and 4 (Pt wire and graphite foil) with the $I-U$ curve for pristine graphite foil with no CCNTs. Figure 1 (b) shows emission current measurements at different temperatures for bare graphite foil.
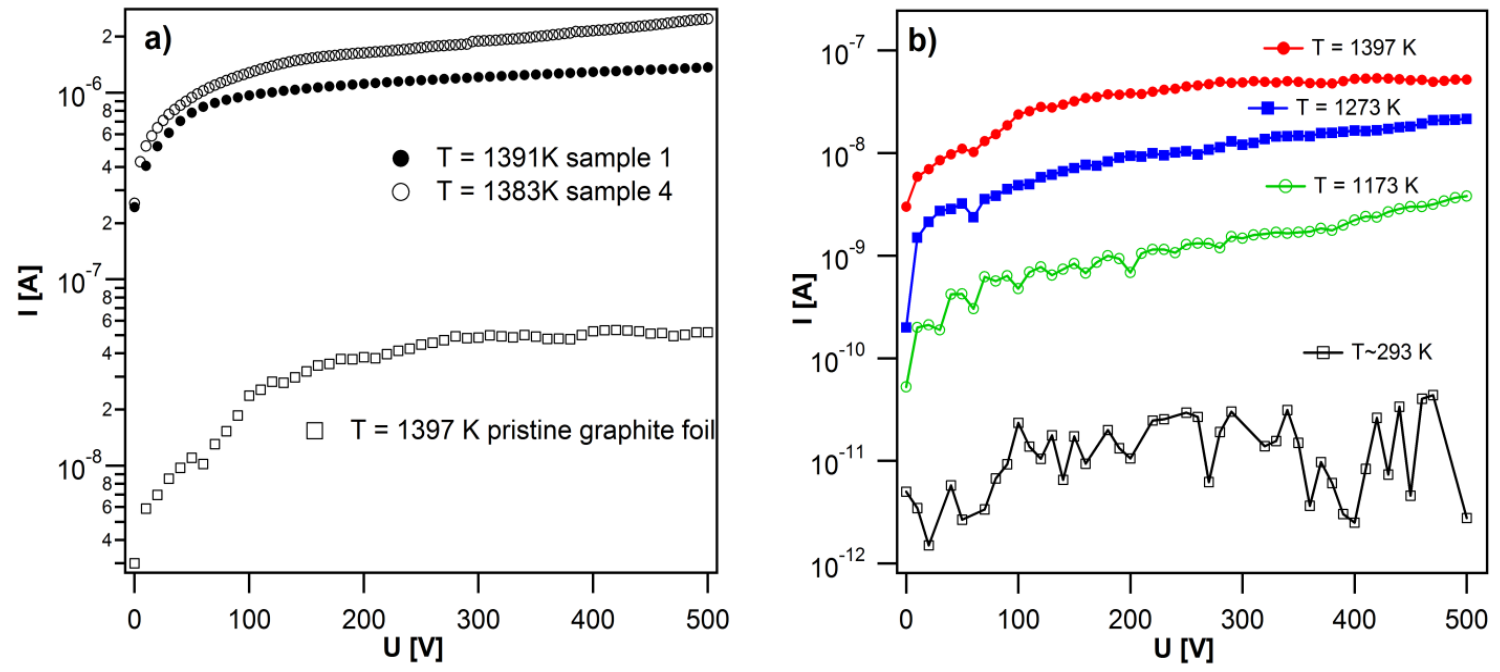

Figure 4.12 $I-U$ characteristic at different temperatures for bare graphite foil and CCNTs on graphite: a) comparison of emission properties between bare graphite and CCNT on graphite foil at same temperature in logarithmic $I$ scale; b) emission properties for bare graphite foil at different temperatures. (Used with permission - Apendix A). 
At maximum attainable temperature, $T=1397 \mathrm{~K}$ maximum value of the current is $52 n A$ for applied voltage $U=500 \mathrm{~V}$. This is an order of magnitude smaller than the emission current for CCNTs under similar conditions.

In an attempt to estimate the quality of the electric contact from the tips of the CCNTs to the substrate - particularly graphite foil. We performed measurements in order to determine $\mathrm{I}-\mathrm{V}$ characteristic of the bare $\mathrm{CCNT}$ using the same experimental set up as for the thermionic emission. The studied sample has CCNTs with an average height about 10-20 $\mu \mathrm{m}$ which are vertically aligned on surface of the foil. Considering that micromanipulator with attached molybdenum anode has accuracy of $\sim 10 \mu \mathrm{m}$ and the fact that our CCNT sample serves as a cathode.

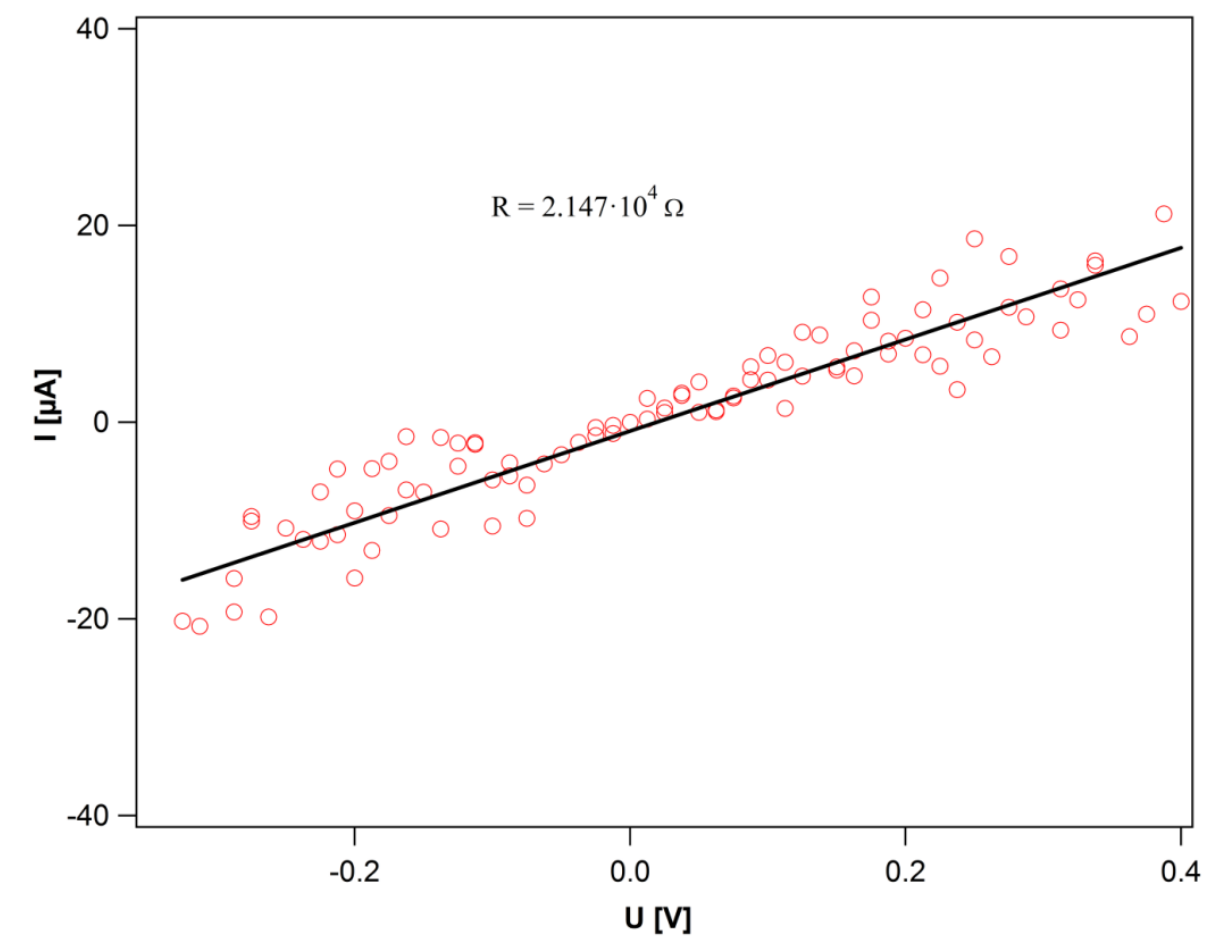

Figure 4.13 I-U characteristic of the array of CCNT grown on the graphite foil in anode-CCNT contact mode. (Used with permission - Apendix B). 
It is possible to make a contact only with CCNTs (not graphite foil) by reducing in small steps sample-anode separation distance and monitoring the change of the current with applied low bias $(\sim 1 \mathrm{~V})$. When the contact is made value of the current would increases usually by one or two orders. Results are presented in Figure 4.12.

From Figure 4.12 it is evident that it forms an Ohmic contact. However measured high value of the resistance $20 \mathrm{k} \Omega$ is probably related to imperfect electric contact between anode and CCNTs. Study of the field emission from CCNTs arrays grown on platinum and graphite foil substrates showed that they are efficient emitters comparable with the vertically grown carbon nanotubes. This fact itself would imply that CCNTs grown on the graphite foil have a good electric contact with substrate and themselves have good electric properties.

\subsection{Summary.}

In summary, CCNT samples were synthesized on Pt wire and graphite foil with different densities, tips sizes, lengths, and wall structures. The work function $\Phi$ was determined by thermionic emission, while the range of $\Phi$ values for each sample could be indirectly related to the morphological characteristics such as aspect ratio, density, and wall structure of CCNTs. The observed lower values for $\phi$. are significantly smaller than reported for multi-walled carbon nanotubes (MWNTs). 


\section{CHAPTER 5}

\section{THERMIONIC EMISSION FROM PHOSPHOROUS DOPED DIAMOND CRYSTALS SUPPORTED BY CONICAL CARBON NANOTUBES. UPS MEASUREMENTS OF THE CVD DIAMOND FILM}

In this chapter we report results of the study of the thermionic emission properties of conical carbon nanotubes grown on the graphite foil coated with $\mathrm{P}$ doped diamond crystals and UPS measurements of the P doped diamond film grown on the $\mathrm{Si}$. We discuss the results of the thermionic emission and UPS measurements and propose the possible mechanisms standing behind determined values of the work function.

\subsection{Synthesis of the $P$ doped diamond crystals and diamond film.}

\subsubsection{Introduction}

We address the challenge of obtaining n-type conductivity in diamond through doping of individual diamond microcrystals which due to increased surface area can be expected to exhibit very interesting overall conductivity values owing to size effects. Such studies can accurately be performed using appropriate architecture allowing the synthesis of individual diamond crystals. Here, we present a novel architecture consisting 
of diamond nanocrystals grown on the tips of conical carbon nanotube (CCNT) array $[69,70]$ and the results of thermionic emission properties. CCNT backbone is expected to provide conducting pathways for diamond crystals. Hence, vertically grown CCNTs allow synthesis of the diamond crystals with increased surface area as well serve as an efficient conducting channels. In addition, work function of undoped and phosphorus doped diamond films were also studied using ultraviolet photoelectron spectroscopy (UPS) for comparison.

\subsubsection{Experimental procedure. Synthesis of the diamond crystals supported by CCNTs and diamond films on Si substrates.}

CCNTs coated with diamond crystals were grown using AsTeX 5010 microwave plasma reactor $(1.5 \mathrm{~kW})$ - the same reactor which hwas used for the synthesis of the CCNT. The CCNT arrays synthesized on graphite foils [70,71] were used as the substrate for nucleation and growth of diamond crystals. These foil substrates were pretreated in ultrasonic diamond solution bath (0-1micron powder in acetone solution) for few seconds followed by cleaning with acetone. The foils were then immersed vertically into the

plasma as shown in Figure 1(a) for the growth of diamond, similar to the growth of CCNT arrays [71] except the height of the foil substrates in this case was lowered towards the diamond growing regime inside the reactor. Di-tertiary butyl phosphine was used as the liquid source for the phosphorus doping. The schematic of the bubbler set up was shown in the Figure 1(b). Hydrogen was used as a carrier gas for the doping precursor. 


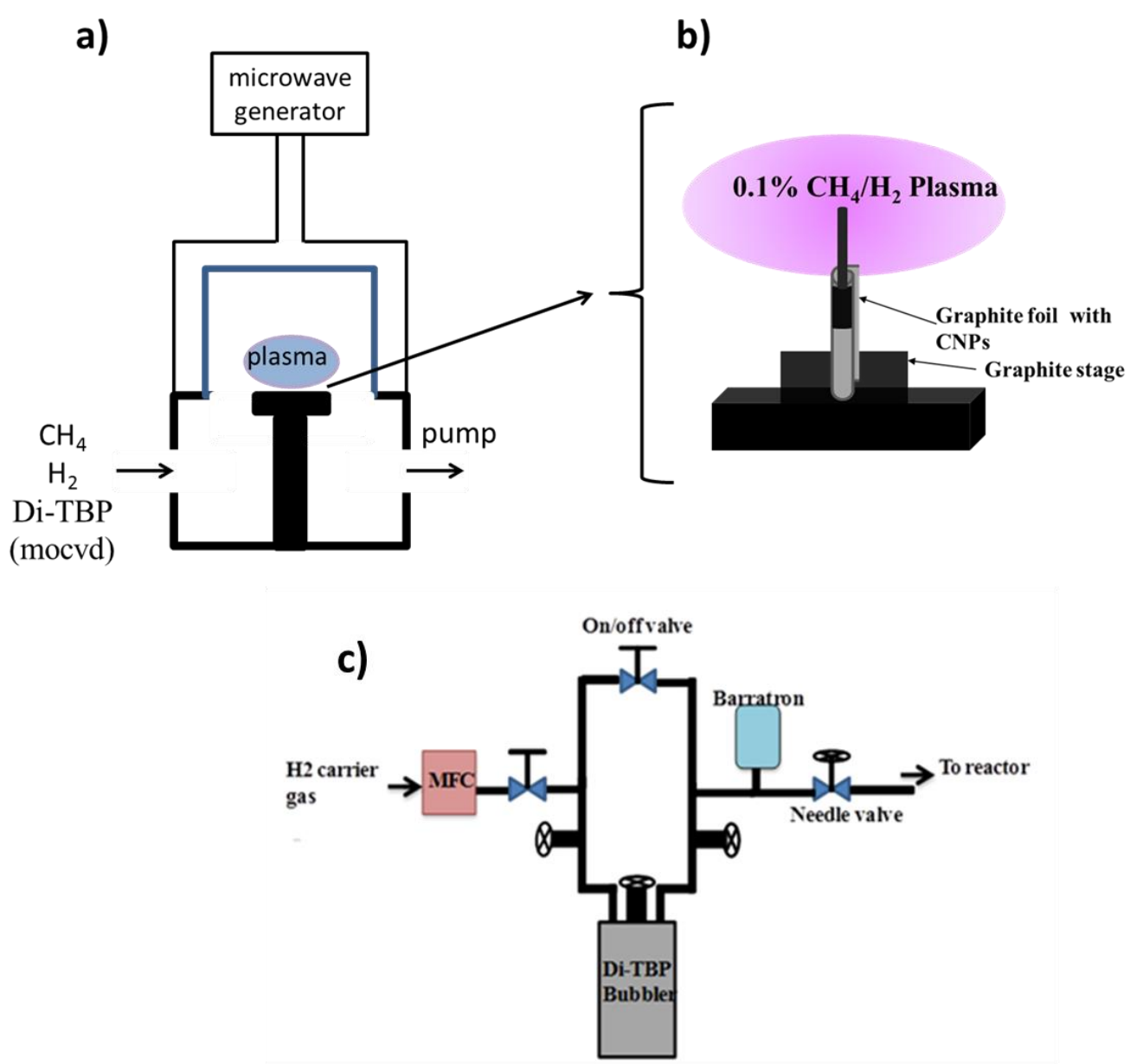

Figure 5.1. Experimental set up for growth of diamond crystals on CCNT arrays: a) vacuum chamber; b) susceptor with grahite foil illustrating sample arrangement; c) bubbler set up for phosphorus doping. (Used with permission - Apendix B).

Depositions were performed at microwave power of 900-1000W, pressures of 30-50 torr with $0.2-1.5 \%$ methane in hydrogen with total flow rate of $200 \mathrm{sccm}$. The phosphorus concentration was varied from $8000-30,000 \mathrm{ppm}$ (with vapor pressure of Di-TBP being measured as 1.7 torr), varying the bubbler pressure and carrier gas (hydrogen) flow rate. Estimated phosphorous/carbon ratio during growth is $0.001-0.004$. The duration of the experiments lasted from $12 \mathrm{~min}$ to $1 \mathrm{hr}$. The carrier gas $\left(\mathrm{H}_{2}\right)$ flow rate of $4-15 \mathrm{sccm}$ was used with bubbler pressure of 720 torr. 
Undoped diamond films were grown on silicon wafers pretreated in ultrasonic diamond solution bath mentioned above. The growth experiments were conducted under the conditions of $3-5 \%$ methane in hydrogen $(200 \mathrm{sccm})$ at 60 torr, $1450 \mathrm{~W}$ microwave power and the sample stage at a temperature of $650{ }^{\circ} \mathrm{C}$ for about $10 \mathrm{hrs}$. P-doping of these diamond films were performed using same dopant source as above. The conditions include $0.2 \%$ methane in hydrogen $(200 \mathrm{sccm})$ at $980 \mathrm{~W}$ microwave power and 50 torr pressure. The carrier gas (hydrogen) flow rate of $4 \mathrm{sccm}$ is used while maintaining the bubbler pressure at 460 torr for about 9 hrs. The phosphorous concentration was 28500 ppm and estimated phosphorous/carbon ratio 0.005 .

\subsubsection{Result of the growth. CCNTs coated with diamond crystals and diamond film on Si substrate.}

Figure 3 shows the SEM images of bare CCNTs, CCNTs coated with undoped diamond crystals, and CCNTs coated with P-doped diamond crystals. The size of the undoped and P-doped agglomeration of diamond crystals is in the range of 1-2 $\mu \mathrm{m}$. The presence of the phosphorus in the diamond crystals was confirmed from the Secondary ion mass spectroscopy (SIMS) as shown in Figure 4, but quantitative value cannot be reported reliably owing to the rough analytical surface. Note that the very rough analytical surface results in a uniform P concentration at larger depths rather than a P tail off presumably due to the increase in roughness exposing more of the nanotubes during the profiling. 

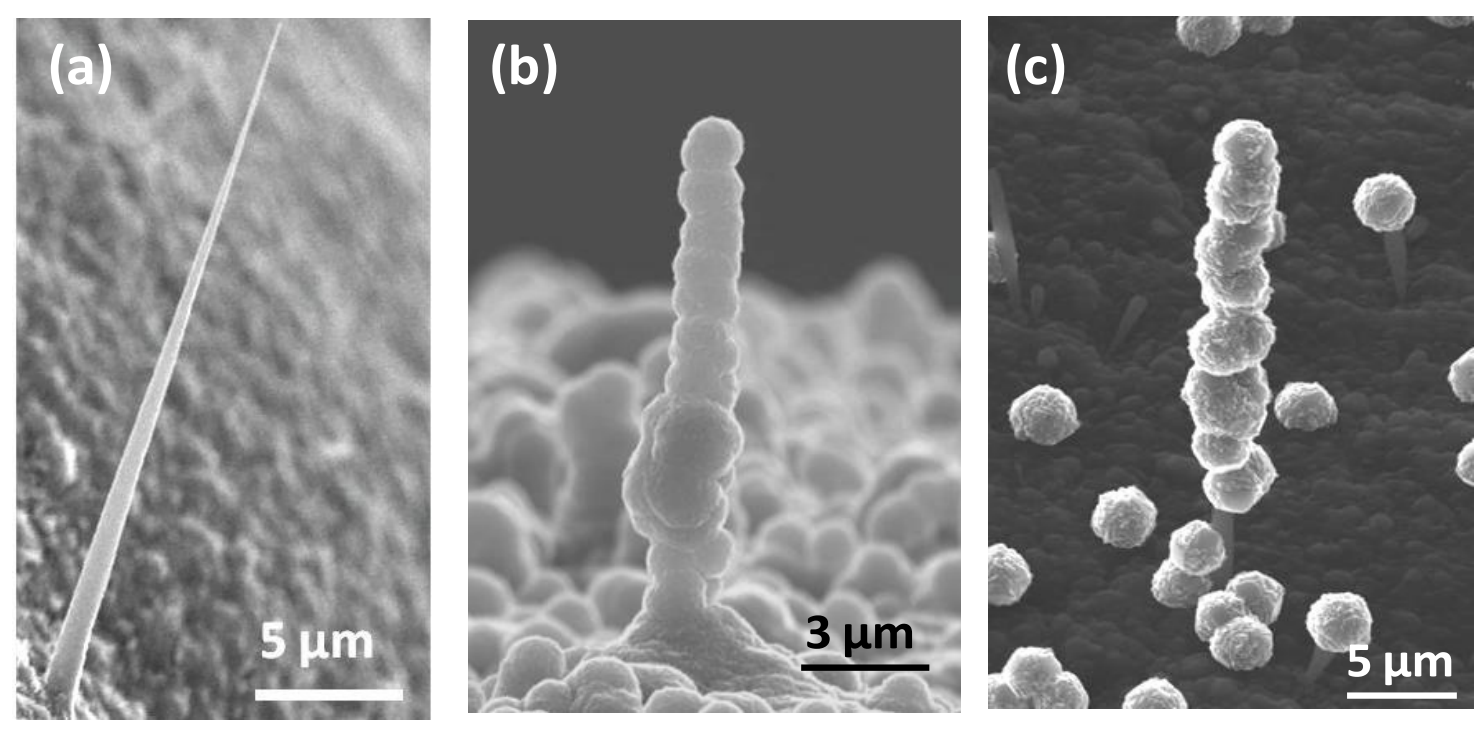

Figure 5.2 Scanning electron microscope (SEM) images of individual a) as synthesized CCNT b) undoped diamond coated CCNT c) P-doped diamond coated CCNT. (Used with permission - Apendix B)

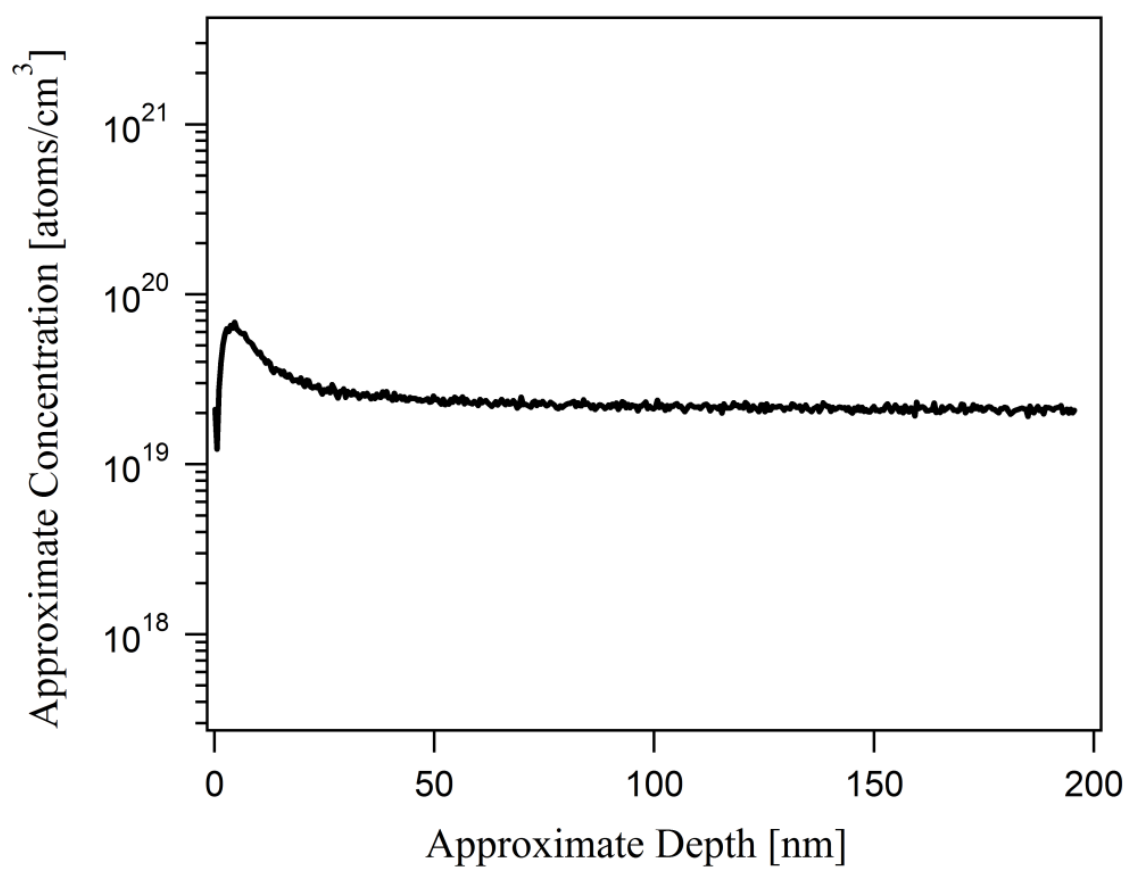

Figure 5.3 Secondary ion mass spectroscopy (SIMS) depth profile of phosphorus in Pdoped diamond crystals on conical carbon nanotubes. Vertical axis represents an approximate concentration. (Used with permission - Apendix B)

The role of the resistivity of the substrate can be mostly ruled out, due to the presence of the conducting substrate (graphite foil) and conducting underlying structures (CCNTs) 
for the diamond emitters. This is advantageous for the emission applications where the resistivity of the substrate plays an important role on the values of emission currents.

In Figure 5.4 SEM images of the P doped the diamond film grown on $\mathrm{Si}$ substrate are shown. As can be seen it is an uniformly grown diamond film with grain sizes ranging approximately $0.5 \mu \mathrm{m}-1 \mu \mathrm{m}$. However it could be seen that the film has significant roughness And as a result SIMS analysis (Figure 5.5) shows a sharp peak for the concentration at the surface as in the case of the P doped diamond on CCNT. Again SIMS data have rather qualitative evidence for the presence of $\mathrm{P}$ impurities in the diamond film.

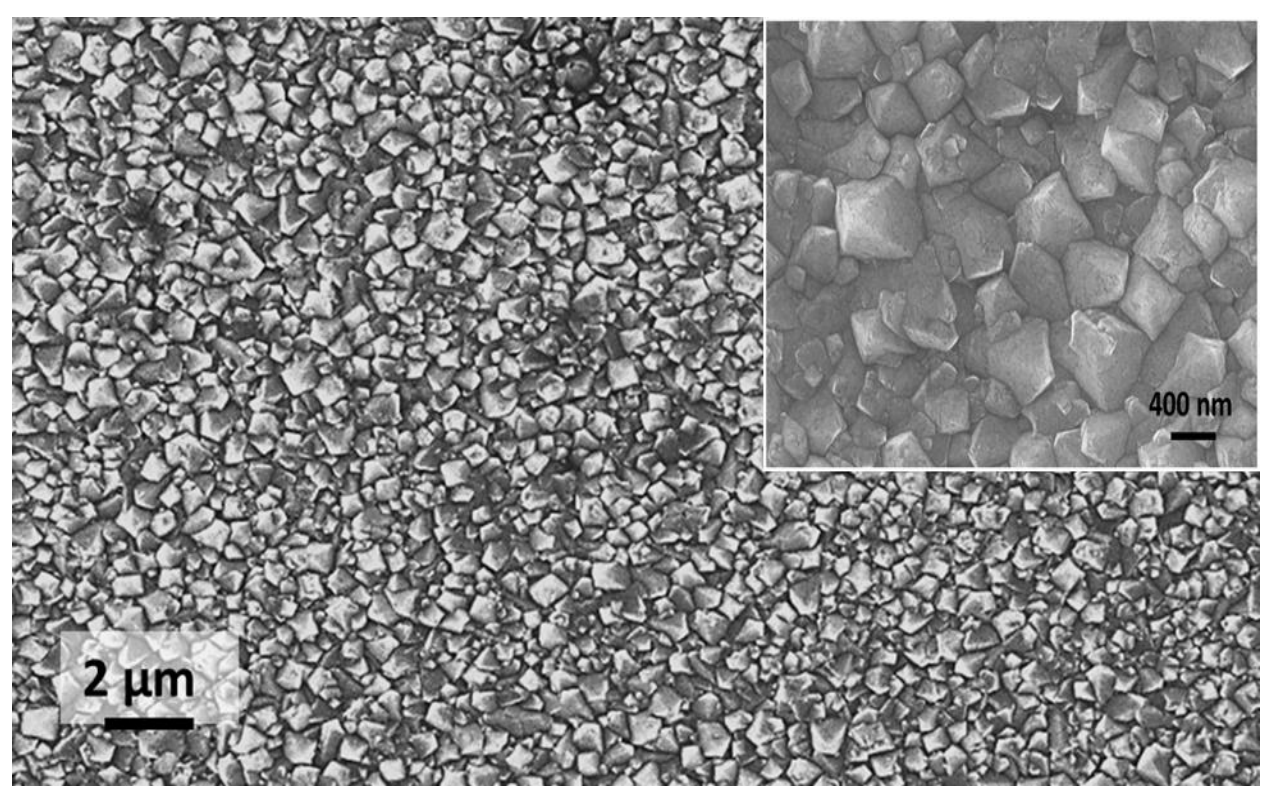

Figure 5.4 SEM images of the P doped diamond film grown on the Si substrate. 


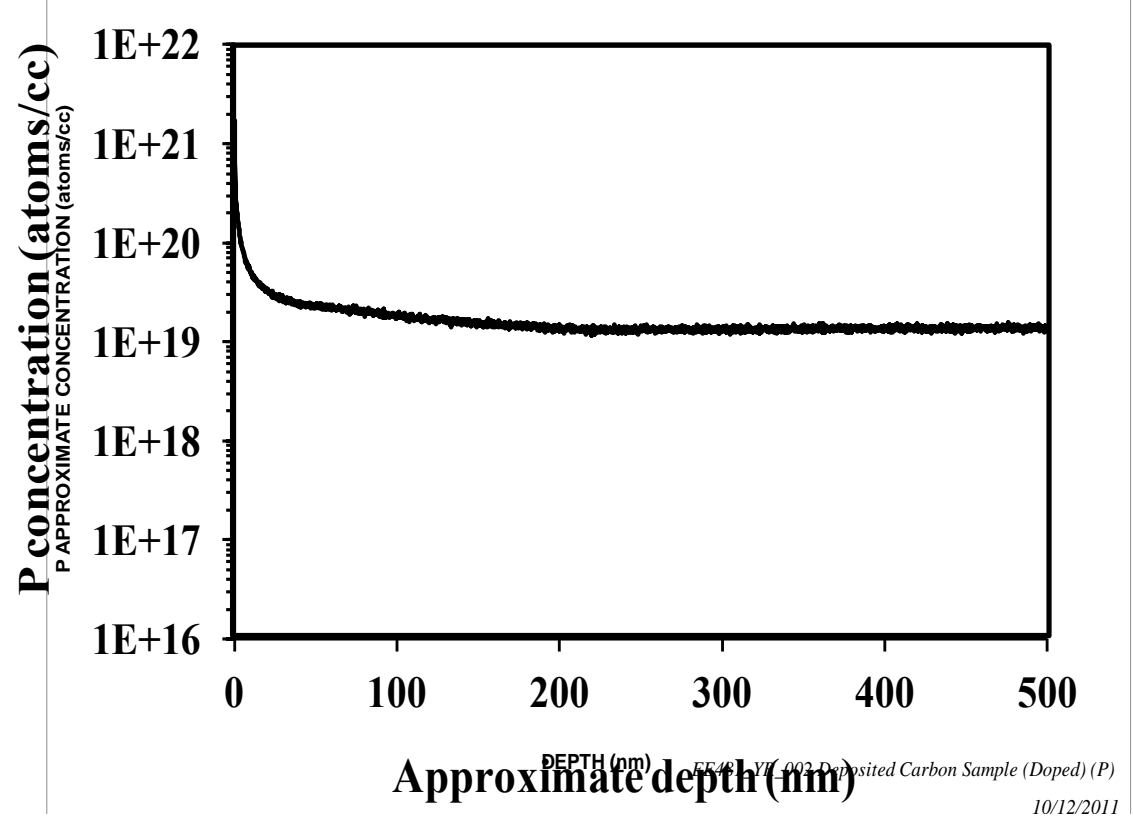

Figure 5.5 Secondary ion mass spectroscopy (SIMS) depth profile of phosphorus in Pdoped diamond films on silicon wafer. (Used with permission - Apendix B).

\subsection{CCNT coated with diamond - Thermionic emission.}

\subsubsection{Thermionic Emission - Experimental.}

Thermionic emission measurements were performed on each sample in a vacuum chamber with base pressure of $\sim 8 \times 10^{-8}$ Torr and $10^{-6}$ Torr at room temperature and 1000 ${ }^{\circ} \mathrm{C}$ respectively. The conducting graphite foil with synthesized CCNT's (or CCNT's with diamond) was placed on a ceramic plate (Boron Nitride) supported on a Pyrolitic BoronNitride $(\mathrm{PBN})$ heater. A conducting molybdenum electrode was attached mechanically to the graphite foil so that the wire and the foil act as the cathode. A molybdenum anode was arranged over the sample and attached to the micromanipulator 
(Figure 4.2). The anode had a shape of the cylinder where the base is facing the sample. Diameter of the anode is $7 \mathrm{~mm}$. Zero distance $(d=0)$ between cathode (sample) and anode was established by observing a sudden electrical short when the anode just touched the sample. Measurements were performed at a set distance, $d$ for different temperatures by sweeping the voltage $U$ from 0 to $500 \mathrm{~V}$ while recording the current $I$ using a picoammeter (Keithley 6487) equipped with a built-in variable voltage source. PBN heater was connected to a separate power supply. Temperature was measured using an infrared pyrometer (Raytek MA2SCCF; Infrared; single color; Spectral response: $1.6 \mu \mathrm{m}$ ).

\subsubsection{Thermionic Emission and work function determination. Results and discussion.}

The work function of the CCNT coated with P doped diamond and undoped diamond was calculated using the same method which we have used to obtain work function values for bare CCNTs. Thus by rearranging and re-plotting thermionic $I-U$ characteristic (Figure 5.6(a)) curves as $\operatorname{Ln}(I)$ vs $\sqrt{U}$ (Figure 5.6(b)), the value of zero field current $I_{0}$ can be extracted for each temperature. The temperature dependence of zero field current, $I_{0}$ is plotted in Figure 5(c), which follows Richardson-Dushman model. Hence the plot of $\operatorname{Ln}\left(\frac{I_{0}}{T^{2}}\right) \quad$ vs. $1 /$ T should result in a straight line (Figure 5(d)) with the slope being $\phi / k T$.

For doped diamond supported on CCNTs a work function value of $\sim 2.23 \mathrm{eV}$ was obtained by this analysis.

Using the same method we obtained work function values for bare CCNT coated with undoped diamond. $I-U$ and $L n(I)-U^{1 / 2}$ curves for bare CCNTs for comparison (Figure 
5.7(a) and (b)) and CCNTs with undoped diamond (Figure 5.7(c) and (d)) are presented at varying temperatures.
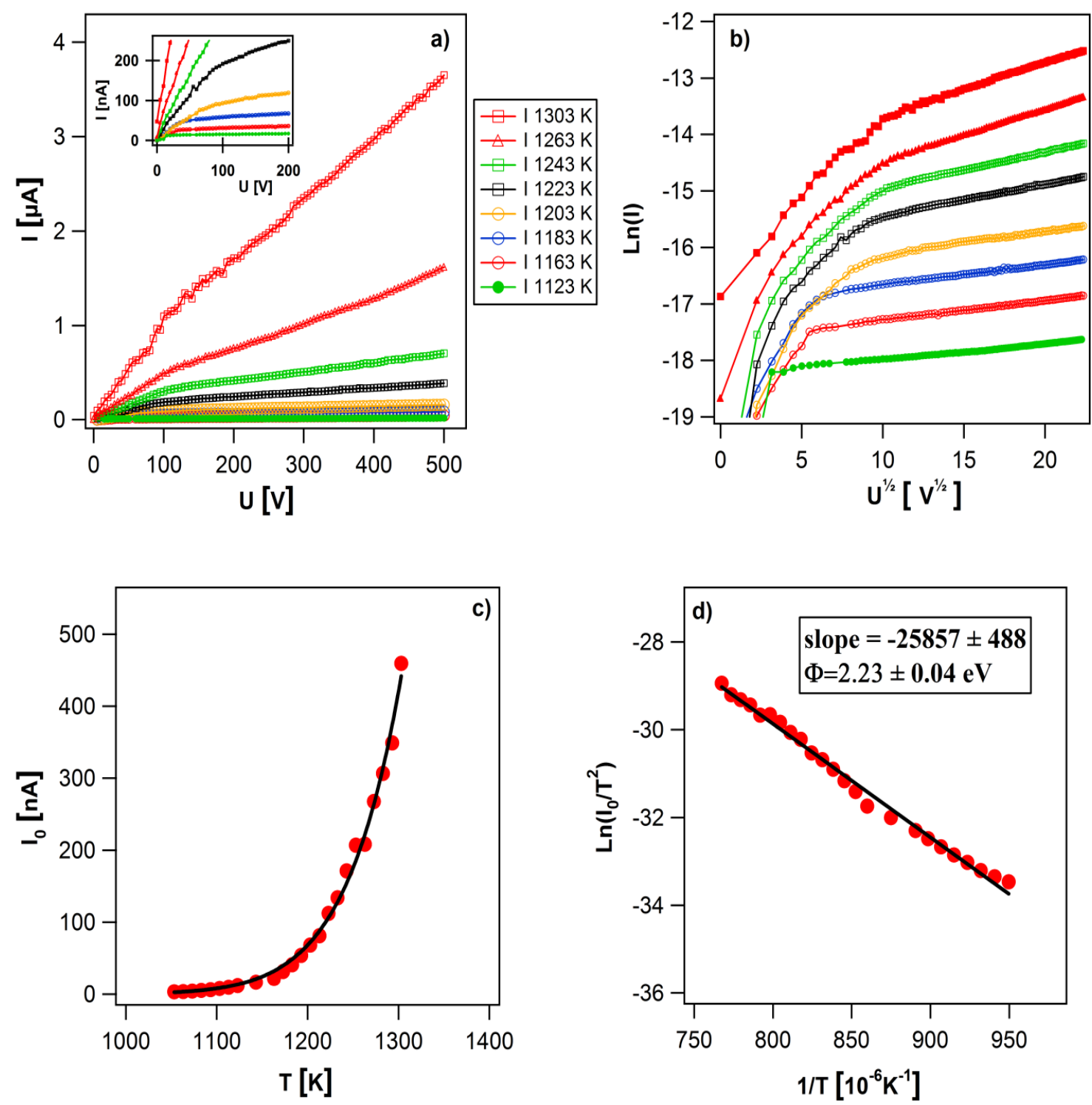

Figure 5.6 P doped diamond crystals on CCNTs: (a) Thermionic I-V characteristics for different temperatures, (b) $\operatorname{Ln}(\mathrm{I})$ vs $\mathrm{U}^{1 / 2}$ (c) Richardson_Dushman curve (d) linear fit of the experimental points - $\operatorname{Ln}\left(\frac{J_{0}}{T^{2}}\right) \mathrm{vs} \frac{1}{T}$. (Used with permission - Apendix B). 

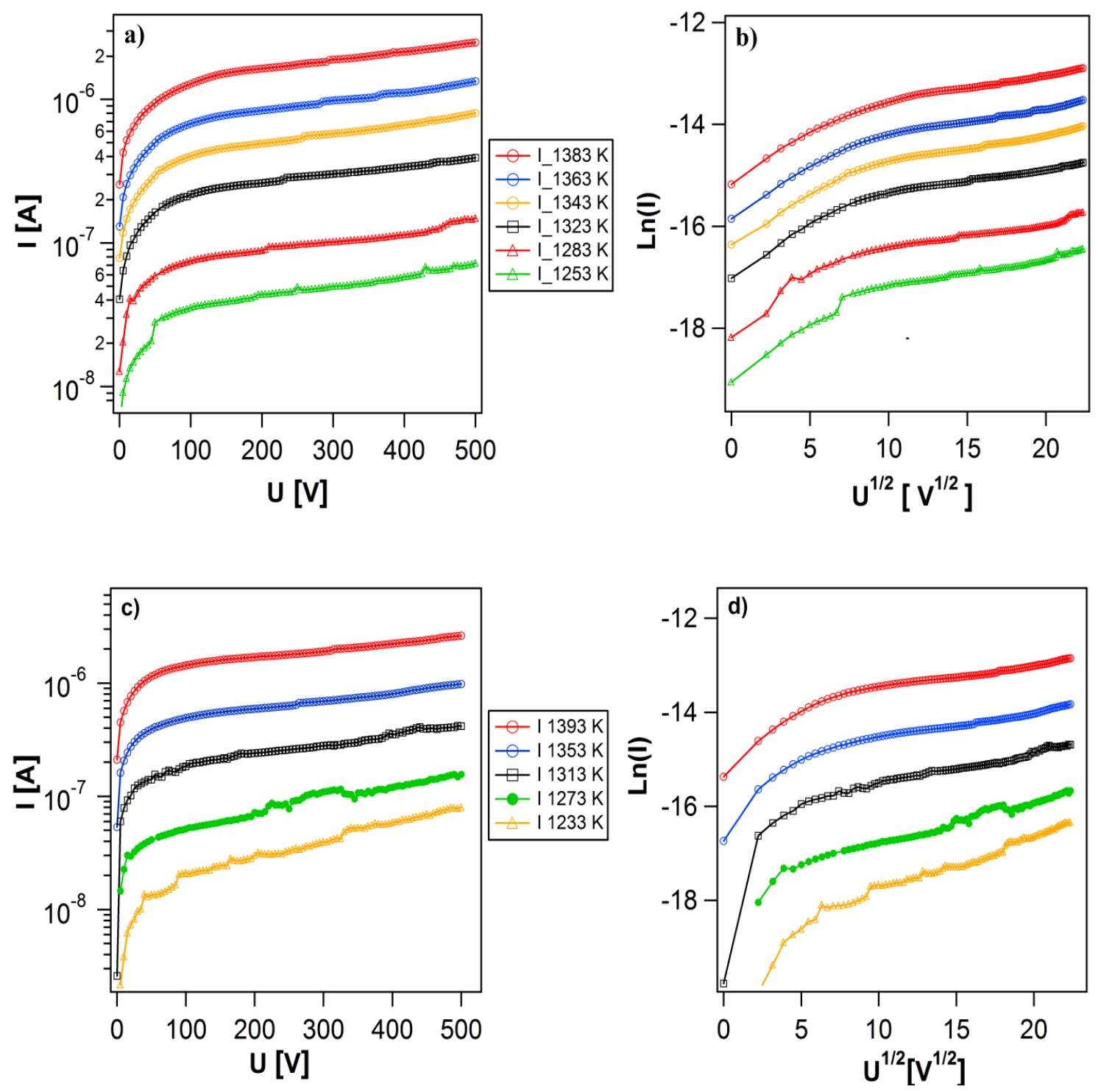

Figure 5.7. Thermionic $I-V$ characteristics and $\operatorname{Ln}(\mathrm{I})$ vs $\mathrm{U}^{1 / 2}$ plots for: $(\mathrm{a}, \mathrm{b})$ bare CCNTs [37] and (c,d) CCNT's with undoped diamond. (Used with permission - Apendix B).

Figure 5.8 summarizes the analysis of Richardson-Dushman formalism for all three samples. The work function values obtained for bare CCNTs, undoped diamond coated CCNTs and P-doped diamond coated CCNT are $4.1 \mathrm{eV}$ [71], $4.26 \mathrm{eV}$ and $2.23 \mathrm{eV}$ respectively. The temperature $(1053 \mathrm{~K})$ at which we were able to observe emission current in case of P-doped diamond is lower than for the other two samples, which could be attributed to its lower work function value. Considering the diamond crystals at the 
tips being the emitting area, the experimental value of the emission constant $A$ is 0.14 $\mathrm{A} / \mathrm{cm}^{2} \mathrm{~K}$. The maximum obtained value of current density is $575 \mu \mathrm{A} / \mathrm{cm}^{2}$ at temperature $1303 \mathrm{~K}\left(1030{ }^{\circ} \mathrm{C}\right)$.
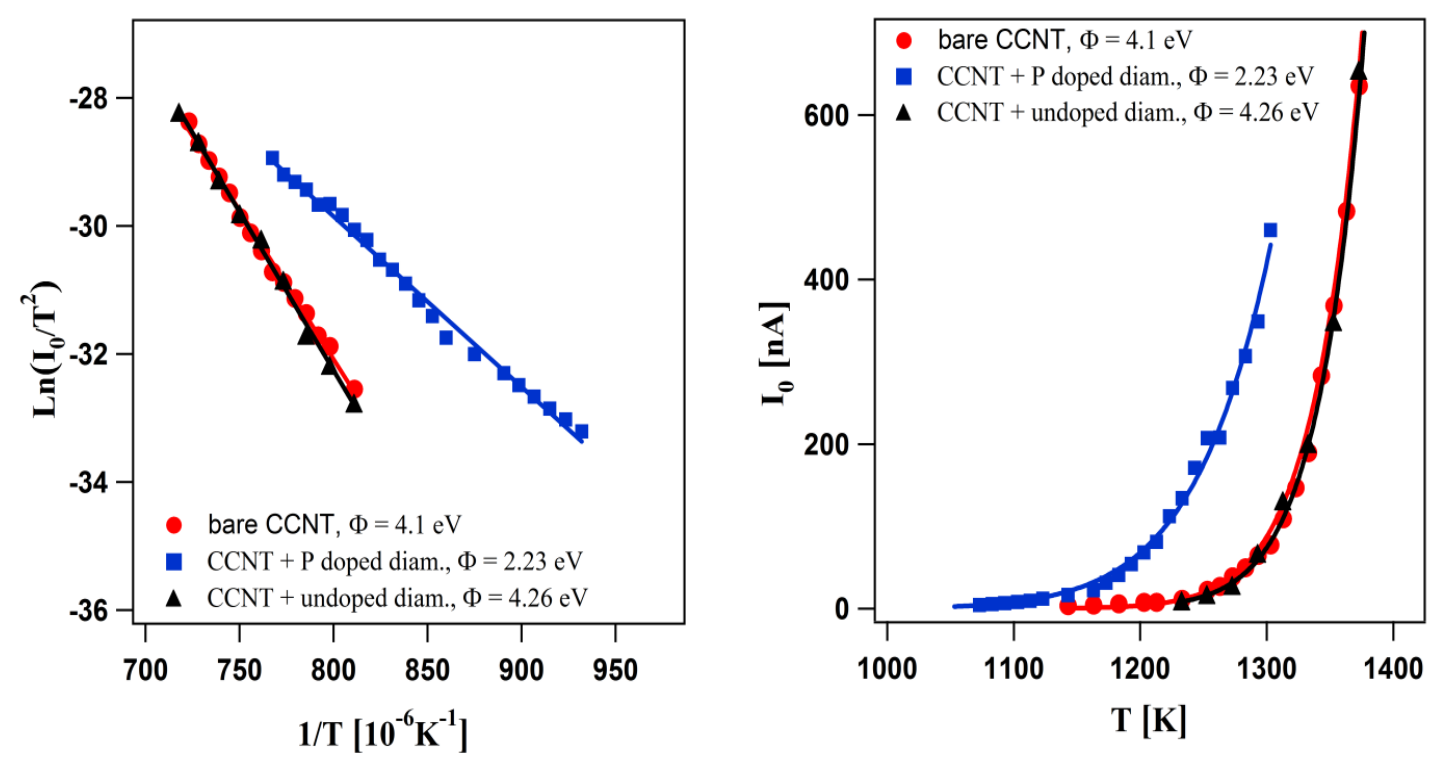

Figure 5.8. Thermionic emission data (dots) and data-fit (line) to Richardson-Dushman equation from thermionic emitters based on bare CCNTs (red), CCNT coated with undoped diamond (green) and CCNT coated with phosphorus doped diamond ( blue). (Used with permission - Apendix B).

It is clearly evident that phosphorus doped diamond has significantly lower work function value compared to bare CCNT arrays and undoped diamond coated CCNT arrays. However, reported value of the work function for P doped diamond film obtained by thermionic emission method $-0.9 \mathrm{eV}$ [62] is significantly lower than ours. The main reason for higher value of work function for the CCNTs coated with P doped diamond is the loss of NEA during the measurements at high temperatures. Previous studies have shown that NEA is lost in the temperature range of $700{ }^{\circ} \mathrm{C}-900{ }^{\circ} \mathrm{C}$ [62]. Minimum temperature at which we were able to measure field enhanced thermionic emission current was about $780{ }^{\circ} \mathrm{C}$ for $\mathrm{P}$ doped diamond sample. Thus reduction of the work function is attributed most probably only to the raising of the Fermi level closer to the 
conduction band from donor states of phosphorus. However in order to obtain a clearer picture, further assumptions needed to be made.

As we mentioned before in chapter 3, previous studies have shown that highly $\mathrm{P}$ doped diamond (face (111)) has upward band bending of $3.2 \mathrm{eV}$ toward surface [53]. The main factor causing such large value was the position of the surface $\mathrm{E}_{\mathrm{Fs}}$, found to be $1.9 \mathrm{eV}$ and $1.6 \mathrm{eV}$ from the valence band maximum at the surface $\left(\mathrm{VBM}_{\mathrm{s}}\right)$ for $\mathrm{H}$ terminated and $\mathrm{O}$ terminated surfaces respectively of $\mathrm{P}$ doped diamond, which is lower than the bulk $\mathrm{E}_{\mathrm{F}}$ situated $0.6 \mathrm{eV}$ below $\mathrm{CBM}$. Surface $\mathrm{E}_{\mathrm{Fs}}$ position is determined by the characteristic surface states, which depend on the amount and nature of the surface defects. It was inferred that diamond surface defects have graphitic nature with charge neutrality point (CNP) estimated to be $1.4 \mathrm{eV}$ above VBM [53,39].

Depending on the type of doping, surface Fermi level can be shifted relatively to CNP. Thus the mechanism of upward bending toward the surface for P doped diamond (in general $\mathrm{n}$ type) was explained qualitatively as the surface $\mathrm{E}_{\mathrm{Fs}}$ shifting and pinning caused by surface defects in the form of graphitic patches or $\mathrm{sp}^{2}$ bonded carbon atoms $[14,39]$. SEM images show highly defective surfaces of diamond crystals grown on CCNTs. We can apply this mechanism of band bending in our case (diamond on CCNTs and diamond film) under the assumption that the approximate position of the surface $\mathrm{E}_{\mathrm{Fs}}$ for $\mathrm{P}$ doped diamond to be $\sim 1.8-2.0 \mathrm{eV}$ above VBM.

Figure 8 shows the band diagrams of diamond surface constructed in order to explain the possible scenarios according to the measured values of work functions of the diamond coated CCNTs. For that purpose, in addition to the position of the Fermi level, it is needed to know the values of the positive electron affinity (PEA) and the band bending $\eta$. It has been reported that donor level for $\mathrm{P}$ in diamond is $0.6 \mathrm{eV}$ below CBM in the bulk 
[48]. The value of PEA for clean diamond surfaces has been reported to be about $0.38-0.7$ $\mathrm{eV}[42,43]$ for a nod doped diamond while the band gap $(\Delta \mathrm{E})$ is $\sim 5.5 \mathrm{eV}$. Undoped polycrystalline diamond films have $\mathrm{p}$ type properties with the bulk $\mathrm{E}_{\mathrm{F}}$ lying $\sim 1 \mathrm{eV}$ above VBM [21].

a)
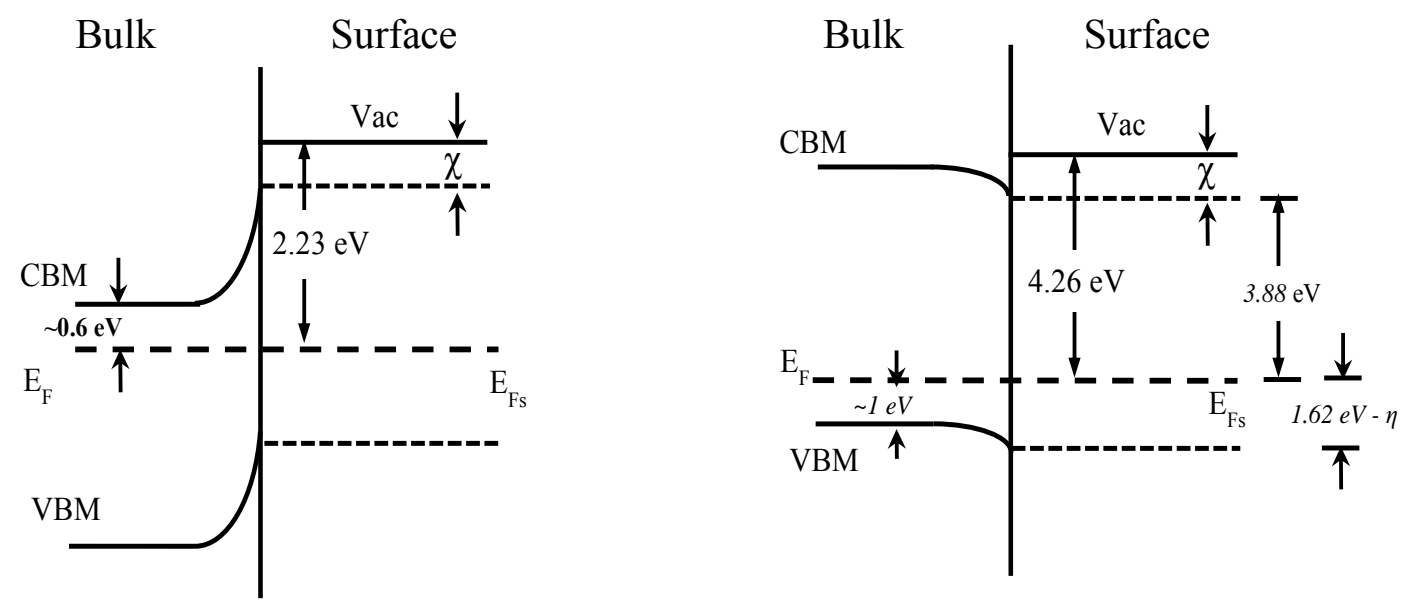

$$
\begin{aligned}
& \mathrm{CBM}-\mathrm{E}_{\mathrm{Fs}}=2.23-\chi \\
& 0.6+\eta=2.23-\chi
\end{aligned}
$$

$$
\mathrm{CBM}-\mathrm{E}_{\mathrm{Fs}}=3.88 \mathrm{eV}
$$$$
\mathrm{E}_{\mathrm{Fs}}-\mathrm{VBM}=1.62 \mathrm{eV}-\boldsymbol{\eta}
$$

b)
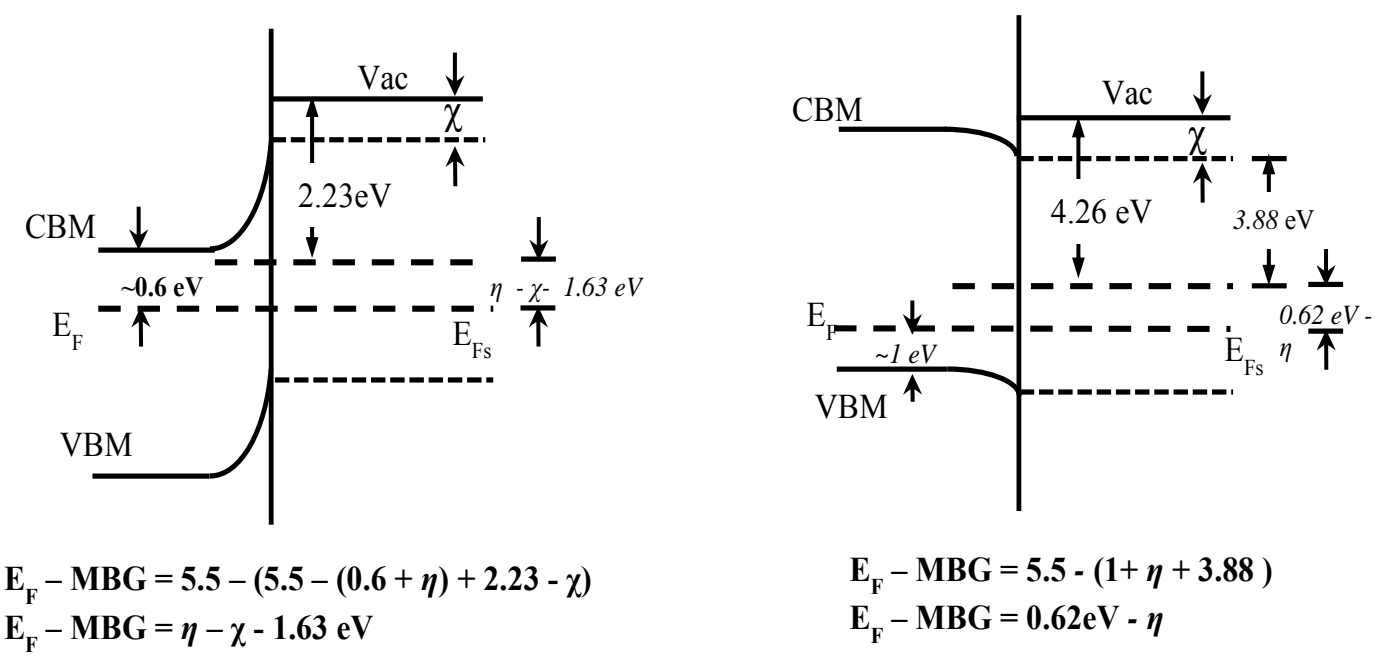
c)

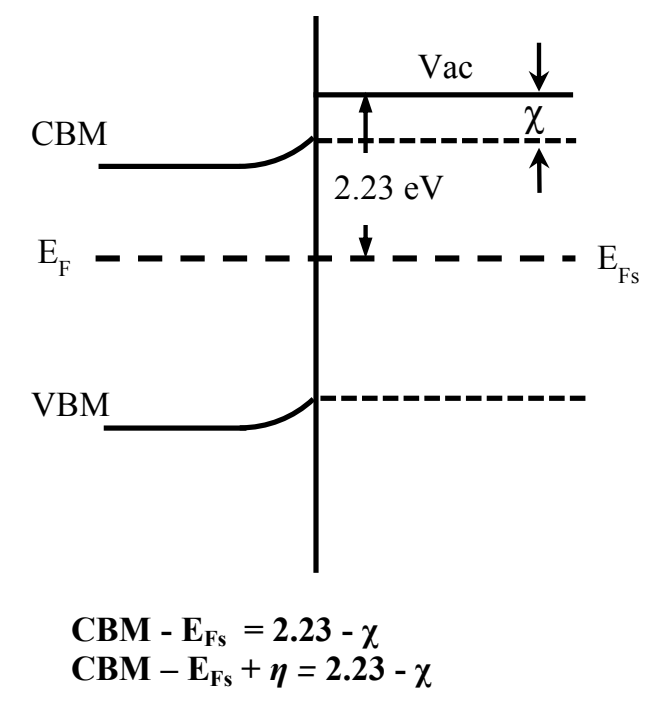

Figure 5.9. Surface energy band diagram of $P$ doped and undoped diamond grown on CCNT's: a) no midband-gap states - work function value comes from position of Fermi level only; b) presence of the midband-gap state; c) only for CCNTs with P doped diamond - bulk Fermi level is much lower in the band gap and there is weak band bending. By short dashed line denoted conduction band minimum $\left(\mathrm{CBM}_{\mathrm{s}}\right)$ and valence band maximum $\left(\mathrm{VBM}_{\mathrm{s}}\right)$ at surface. By long dashed line denoted Fermi level and midband-gap state (MBG). (Used with permission - Apendix B).

We propose three possible scenarios which result in obtained value of work function for P doped diamond grown on CCNTs stems: first is the pinning of the bulk Fermi level to the surface Fermi level and resulting in band bending. In second case could be considered combination of the pinning and the presence of the midband-gap (MBG) state originated from defects (due to doping and morphology of the individual diamond crystals) (Figure 5.9) [21]. Finally the third possibility (regarding only P doped diamond coated CCNTs) is that presence of the defects and impurities could cause lowering of the bulk Fermi level. As an additional effect - surface states could be compensated by the donors and defects. Based on the band diagrams we can give more detail description of 
possible cases for P doped diamond on CCNTs which can result in such values of work function:

(i) there is no midband-gap states (or it's overlapping with Fermi level), which results probably in lower value of upward band bending $\eta=1.63-\chi$ than it was reported for $\mathrm{P}$ doped diamond. Position of surface Fermi level $\mathrm{E}_{\mathrm{Fs}}$ below conduction band minimum at the surface $\left(\mathrm{CBM}_{\mathrm{s}}\right)\left(\mathrm{CBM}_{\mathrm{s}}-\mathrm{E}_{\mathrm{Fs}}=2.23-\chi\right)$ would imply low amount of surface graphitic defects;

(ii) If we assume the presence of the midband - gap states for CCNTs coated with P doped diamond crystals we can define energy difference between MBG and Fermi level at the surface which depends on the value of the band bending $\eta$

$$
\begin{aligned}
& \mathrm{E}_{\mathrm{F}}-\mathrm{MBG}=\Delta \mathrm{E}-\left[\left(\mathrm{E}_{\mathrm{Fs}}-\mathrm{VBM}\right)+(\mathrm{CBM}-\mathrm{MBG})\right] \\
& \mathrm{E}_{\mathrm{F}}-\mathrm{MBG}=5.5-[(5.5-(0.6+\eta))+2.23-\chi]=\eta-\chi-1.63 \mathrm{eV}
\end{aligned}
$$

and for CCNTs coated with undoped diamond

$$
\begin{aligned}
& \mathrm{E}_{\mathrm{F}}-\mathrm{MBG}=\Delta \mathrm{E}-\left[(\mathrm{CBM}-\mathrm{MBG})+\left(\mathrm{E}_{\mathrm{Fs}}-\mathrm{VBM}\right)\right] \\
& \mathrm{E}_{\mathrm{F}}-\mathrm{MBG}=5.5-(3.88+1+\eta)=0.62 \mathrm{eV}-\eta
\end{aligned}
$$

Based on the previous reports about the position of pinned Fermi level for P doped diamond - if $\mathrm{E}_{\mathrm{Fs}}-\mathrm{VBM}=1.6 \mathrm{eV}$; obtained value of band bending $\eta$ would be $3.86-\chi$, whereas if $\mathrm{E}_{\mathrm{Fs}}-\mathrm{VBM}=2 \mathrm{eV}$ then $\eta=2.9 \mathrm{eV}$.

(iii) Lowering of the bulk Fermi level and raising of the surface Fermi level due to surface (state compensation) would result in weak band bending. In this situation energy difference would be expressed as follows

$$
\mathrm{CBM}-\mathrm{E}_{\mathrm{f}}=2.23-\chi-\eta
$$


Considering weak band bending we can write that $\eta \rightarrow 0$ and $\mathrm{CBM}-\mathrm{E}_{\mathrm{f}}=2.23-\chi$. If we asssume that value of electron affinity at the surface of the doped diamond crystals would be comparable with the value of electron affinity of the clean surface of the undoped diamond $-0.38 \mathrm{eV}-0.7 \mathrm{eV}$. We can conclude that Fermi level could be situated approximately $1.5-2 \mathrm{eV}$ below conduction band minimum.

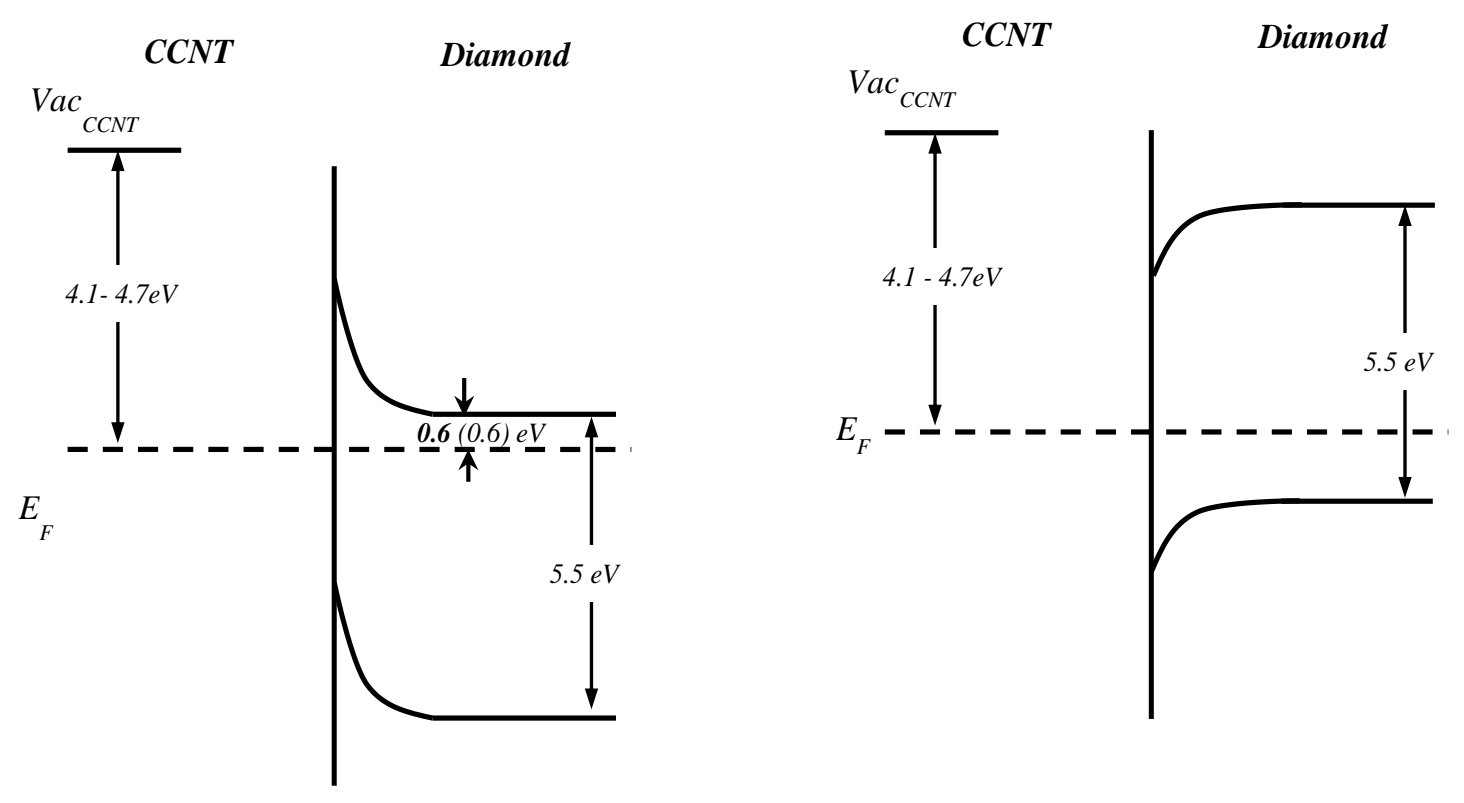

Figure 5.10. Schematic view of band diagram illustrating the band bending at the interface of CCNTs and diamond. (Used with permission - Apendix B).

To complete the picture of the thermionic emission mechanism from our hybrid structurewe must address the fact that at lower temperatures $\left(<800{ }^{0} \mathrm{C}\right)$ we have not observed sufficiently high values of $\mathrm{I}_{\mathrm{FETE}}$ (to be detected) which is the onset of the presence of NEA. We believe that main reason is the development of the potential barrier at the CCNTs and P doped diamond interface due to band bending as shown in Figure 5.10 in analogous to the case of metal/n-type semiconductor structures. Considering the 
CCNTs as the conducting channels for diamond it is needed to attain higher temperatures $\left(>800{ }^{\circ} \mathrm{C}\right)$ to obtain sufficient values of current across the interface to supply electrons for emission.

\subsection{Ultraviolet Photoelectron Spectroscopy of diamond films grown on the Si substrate.}

\subsubsection{UPS - Experimental procedure.}

Here, a multi chamber ultra-high vacuum (UHV) surface science facility (VG Scientific /RHK Technology) comprising of a $150 \mathrm{~mm}$ radius CLAM 4 hemispherical analyzer was used. The base pressure of the chamber was in the $10^{-9}$ torr range. A differentially pumped He-discharge lamp was used as the UV radiation source. The samples for this type of measurement typically have the active material in proper electrical contact with a gold film sputtered on copper foil to equilibrate the Fermi levels $\left(E_{F}\right)$. For that reason a part of the copper foil sputtered with gold was used as the sample and the other half was used for acquiring the reference gold spectra. Double sided copper tape was used to make the electrical contact between silicon substrates with diamond films and the underlining gold film on copper foil. The measurements were performed using He-I (21.23 eV) and He-II (40.81 eV) UV excitations. A stable bias was provided to avoid the instrumental cutoff in the lens system of the analyzer at low kinetic energy (KE) for all the UPS spectra measurements. The external bias was taken into

consideration in order to shift the spectra back to zero-bias position through post 
acquisition data processing. The calibration of the UPS spectrometer was performed by measuring the absolute position of the Fermi level of the gold sample using He-I. A spectrum, measured using a negative bias of $4.9 \mathrm{eV}$ is shown in the Figure 2(a) resulting in an $\mathrm{E}_{\mathrm{F}}$ value of $21.23 \mathrm{eV}$ that is in well agreement with the expected value. Similarly He-II spectrum (Figure 5.11(b)) shows Fermi level value of $40.83 \mathrm{eV}$, which also agrees with the literature value [72]. As the active sample is in electrical contact with the reference gold film, the sample also has the same Fermi level as of the gold. The work function of the material is then determined from the intersection of low-KE cut off tail with the background level.
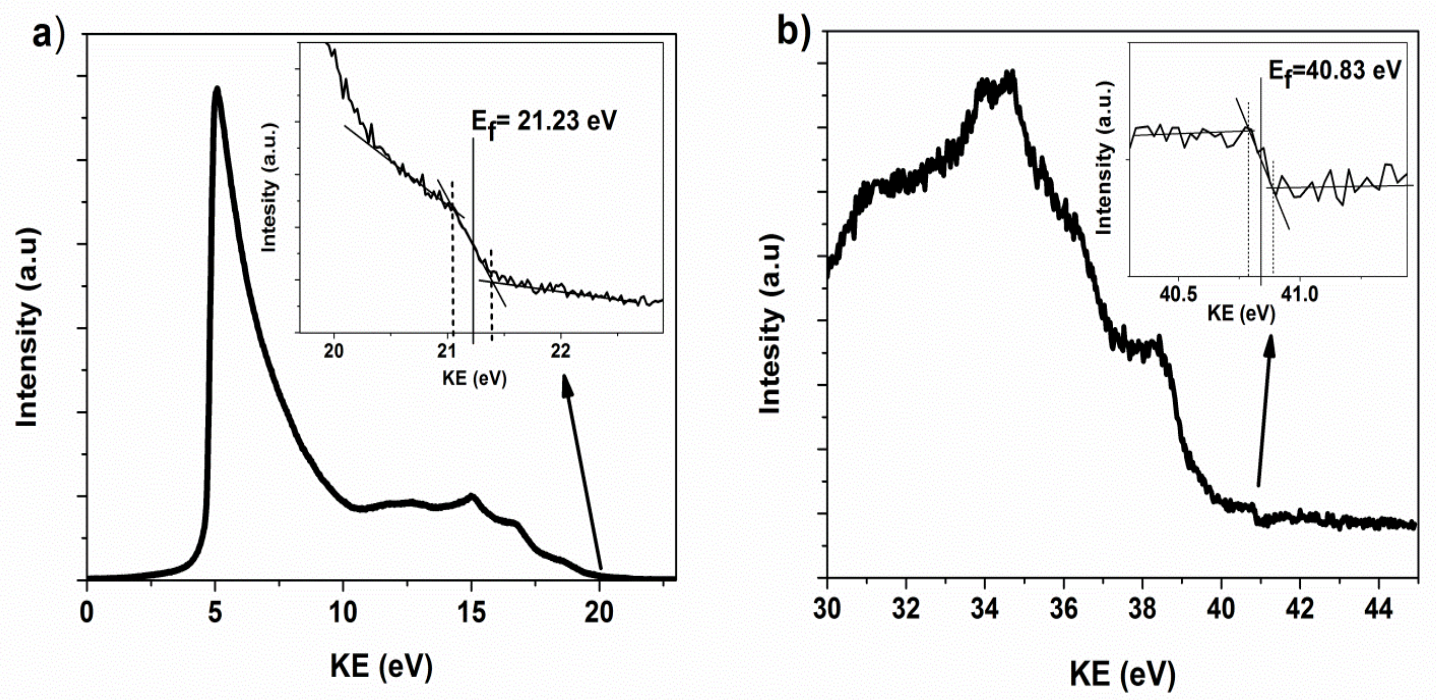

Figure 5.11 UPS spectra of gold film showing Fermi level positions at (a) $21.23 \mathrm{eV}$ for $\mathrm{He}$ I (b) $40.83 \mathrm{eV}$ for He II radiations. Insets show magnified part of UPS spectra with Fermi edge. (Used with permission - Apendix B). 


\subsubsection{UPS and work function determination of the diamond films - \\ Results and discussion.}

We discussed in the chapter 3 that UPS measurements of diamond could provide information about electron affinity and surface Femi level position [80]. In view of the fact that we didn't observe any evidence for NEA in case of CCNTs coated with diamond we turned our attention to $\mathrm{P}$ doped diamond films grown on silicon substrate. Figure 5.12 shows the low kinetic energy part of the He-I emission spectra of (a) undoped and (b) phosphorous doped films. Both undoped and P-doped diamond films exhibit two peaks one for a low KE cut-off and a second high intensity peak characteristic of work function and NEA behavior with electrons coming from $\mathrm{CBM}_{\mathrm{s}}$ respectively [80].
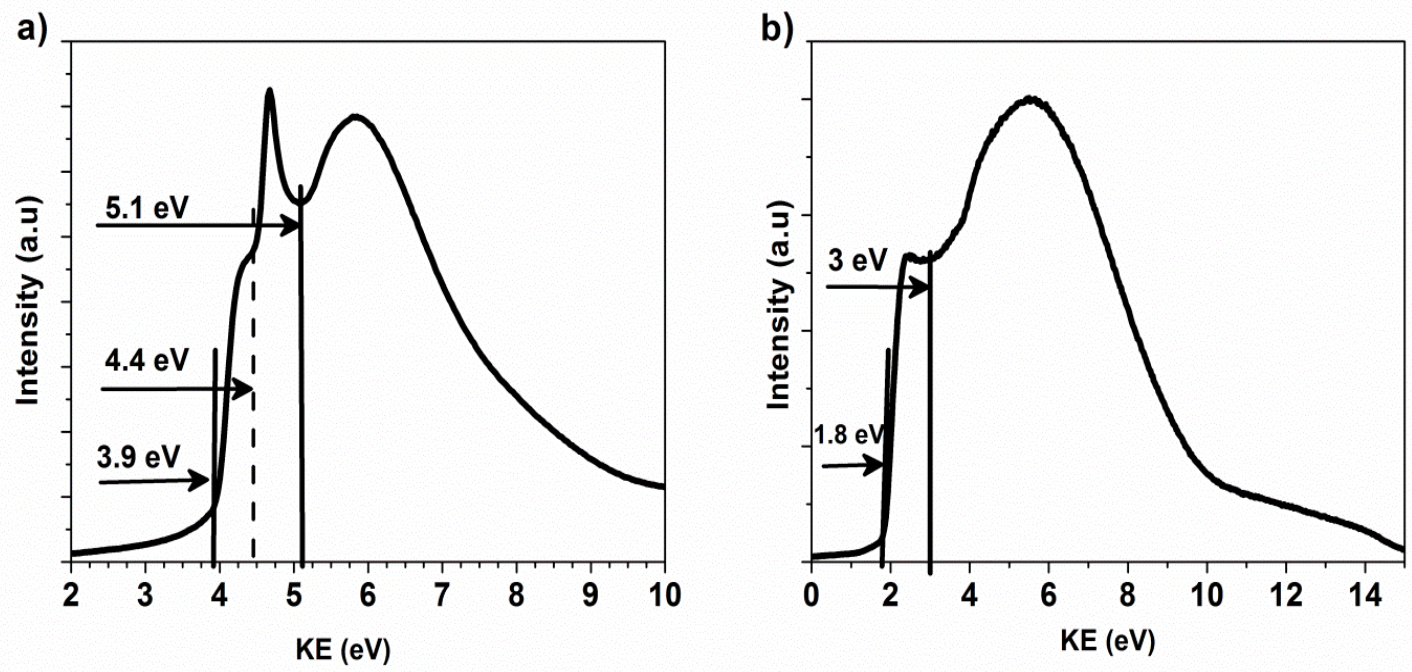

Figure 5.12. Low kinetic-energy part of $\mathrm{He}-\mathrm{I}(21.23 \mathrm{eV})$ spectra of a) undoped diamond film; b) phosphorus doped diamond film. Spectra of the undoped diamond film show low kinetic cut-off energy positions indicative of effective work function, sharp peak related to thermalized electron states and a high intensity cut-off position of conduction band minimum due to the presence of NEA and MBG state. Spectra of the $p$ doped diamond film has no thermalized electrons sharp peak but has visible KE cut-off of eff. work function and NEA high intensity cut-off. (Used with permission - Apendix B). 
All the numbers for the cut off energy positions are obtained with the extrapolation to zero intensity.

In the case of undoped diamond, the value of the effective work function is given as $3.9 \mathrm{eV}$ and sharp peak is related to electrons thermalized to the bottom of conduction band and lower edge of sharp peak defines $\mathrm{CBM}_{\mathrm{s}}$ position to be $4.4 \mathrm{eV}$ above surface Fermi level $\left(\mathrm{CBM}_{\mathrm{s}}-\mathrm{E}_{\mathrm{Fs}}=4.4 \mathrm{eV}\right)$.

The lower energy edge of the second wider peak $5.1 \mathrm{eV}$ could be related to midband-gap state $\left(\mathrm{CBM}_{\mathrm{s}}-\mathrm{MBG}\right)$ which will be discussed further. For P-doped diamond, a low KE cut-off is situated at $1.8 \mathrm{eV}$ and $\mathrm{CBM}_{\mathrm{s}}$ position at surface situated at $3 \mathrm{eV}$ giving rise to same NEA value of $1.2 \mathrm{eV}$. A work function value of $4.67 \mathrm{eV}$ obtained for gold film lies in the value range reported in the literature [72]. Interestingly work function value of $3.6 \mathrm{eV}$ has been reported for hydrogen terminated phosphorus doped (111) single crystal diamond [53]. An upward band bending of $3.2 \mathrm{eV}$ from the simulated surface Fermi level position and absence of NEA from the Secondary Electron Spectra was attributed to the large work function value.

Though the presence of the NEA was predicted theoretically, the reason for the absence could not be evidently articulated [52]. The similar explanation for the lack of NEA peaks in UPS spectrum (band bending towards surface) has been proposed for Ndoped diamond with $\mathrm{H}$ terminated surface [80]. However, in the present case the presence of NEA value of $1.2 \mathrm{eV}$ evident from the UPS spectra could be attributed to the reduced work function value detected. 
a)
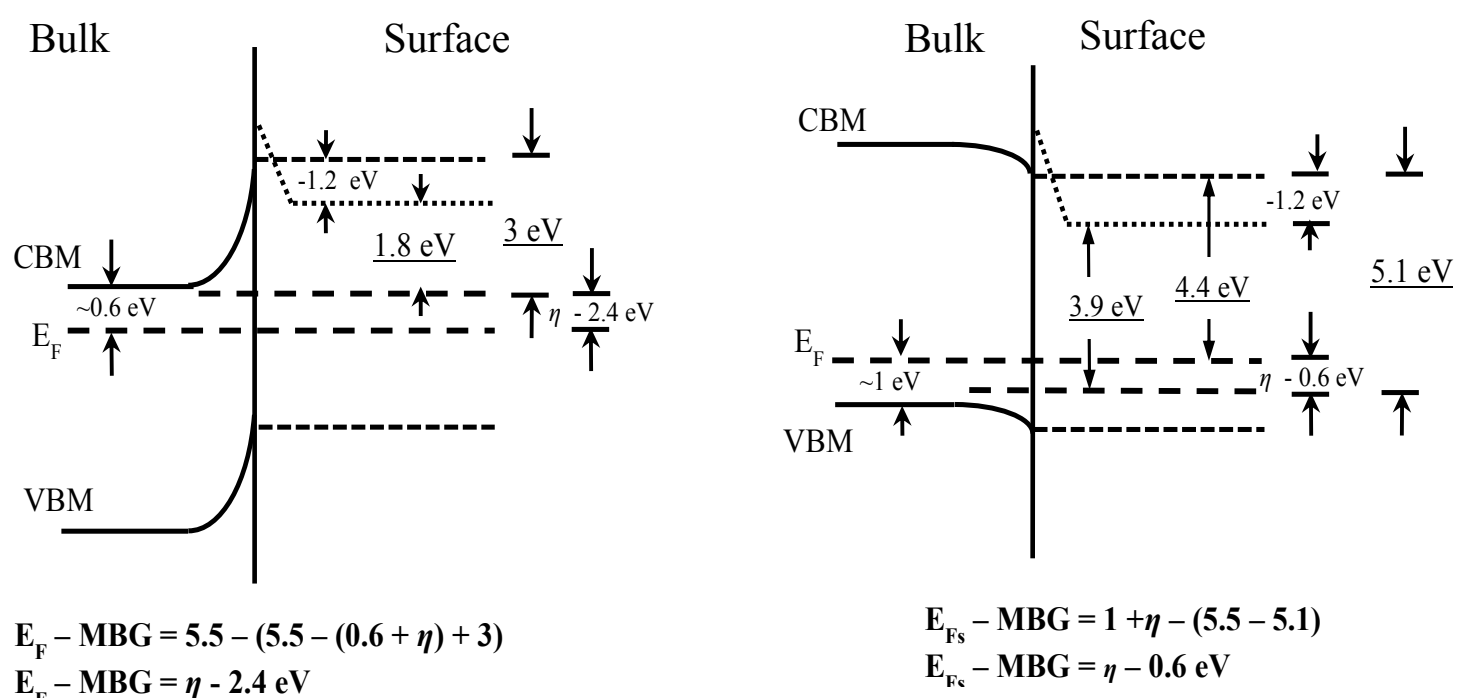

b)

c)
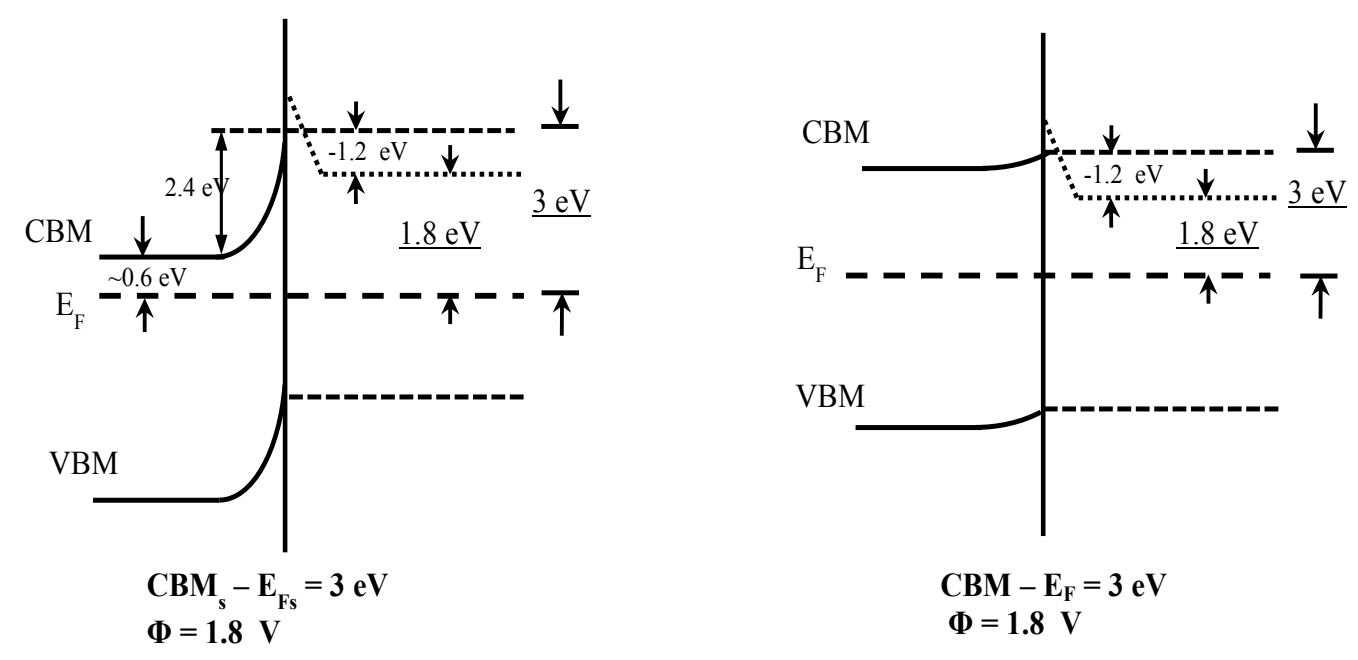

Figure 5.13 Surface energy band diagrams for $\mathrm{P}$ doped and undoped diamond films grown on $\mathrm{Si} / \mathrm{SiO}_{2}$ - based on UPS results: a) presence of the midband-gap state for $\mathrm{P}$ doped and undoped diamond films; b) no midband-gap state for P doped diamond film; c) no midband-gap states, lowering of the bulk Fermi level and weak band bending. Energy difference for undoped diamond was obtained from equation $E_{F}-M B G=\left(E_{F}-V B M\right)$ $[\triangle \mathrm{E}-(\mathrm{CBM}-\mathrm{MBG})]$. Dotted line in both band diagrams is the vacuum level in case of NEA. Underlined values of energies are obtained from UPS measurements. By short 
dashed line denoted conduction band minimum $\left(\mathrm{CBM}_{\mathrm{s}}\right)$ and valence band maximum $\left(\mathrm{VBM}_{\mathrm{s}}\right)$ at surface. By long dashed line denoted Fermi level and midband-gap state (MBG). (Used with permission - Apendix B).

We consider three most probable scenarios for the P doped diamond films and one for the undoped diamond film which are all illustrated in the band diagram (Figure 5.13) constructed based on the UPS results:

(i) if there is present midband-gap state (Figure 11 a)), the energy difference between MBG and surface Fermi level for P doped diamond film and undoped diamond film respectively would be $\eta-2.4 \mathrm{eV}$ and $\eta-0.6 \mathrm{eV}$. In agreement with previous assumption, if Fermi level is at $\sim 2 \mathrm{eV}$ above VBM, in result $\eta-2.4 \mathrm{eV}=0.5 \mathrm{eV}$ and value of $\eta$ in this case is $2.9 \mathrm{eV}$ which is close to the value $3.2 \mathrm{eV}$ reported by Kono et al. [53]. As for the undoped diamond films, from the band diagram we can determine the position of the surface Fermi level at $1.1 \mathrm{eV}$ above VBM. From the equation describing energy difference we know that $\mathrm{E}_{\mathrm{Fs}}-\mathrm{MBG}=0.7 \mathrm{eV}$ and $\mathrm{MBG}$ position is $0.4 \mathrm{eV}$ above $\mathrm{VBM}$.

(ii) Upward band bending at the surface and NEA (no midband-gap states) (Figure 11 b)). Value of work function is $1.8 \mathrm{eV}$, energy difference at surface $\mathrm{CBM}_{\mathrm{s}}-\mathrm{E}_{\mathrm{Fs}}=3 \mathrm{eV}$. Obtained upward band bending value is $2.4 \mathrm{eV}$. Thus higher than for $\mathrm{P}$ doped crystals on CCNTs. For undoped diamond film value of the upward band bending is $\sim 0.6 \mathrm{eV}$ where work function value is $3.9 \mathrm{eV}$ and energy difference $\mathrm{CBM}_{\mathrm{s}}-\mathrm{E}_{\mathrm{Fs}}=5.1 \mathrm{eV}$.

(iii) In the third scenario we could again assume that bulk Fermi level is significantly lower and surface Femi level is higher in the band gap. In consequence there is no or very weak band bending. Based on UPS data Fermi level in this case would be $3 \mathrm{eV}$ below CBM thus indicating the $\mathrm{p}$ type property of the diamond film in the bulk. We did performed thermionic emission measurements from $\mathrm{P}$ doped nanocrystalline diamond film (with hydrogenated surface) grown on W foil (Appendix A, Figure 12) and found 
that the value of effective work function is $1.33 \mathrm{eV}$. Considering the presence of NEA in case of the diamond film grown on $\mathrm{W}$ foil it could imply that value obtained value 1.33 $\mathrm{eV}$ is the difference between the surface Fermi level and $\mathrm{CBM}_{\mathrm{s}}$. Which would support argument about the lower position of the bulk Fermi level.

This is the first report on the work function of phosphorus doped diamond polycrystalline films, and further theoretical calculations are needed to accommodate for all the differences in the present scenarios for the determination of position of the surface Fermi level and band bending values.

\subsection{P doped diamond film on $\mathrm{W}$ foil.}

We also measured thermionic emission from $\mathrm{P}$ doped nanocrystalline film grown on $\mathrm{W}$ foil. $\mathrm{P}$ doped diamond films were synthesized on $\mathrm{W}$ foils pretreated in ultrasonic diamond solution bath. Growth was performed under the conditions of $1.5 \% \mathrm{CH}_{4} / \mathrm{H}_{2}$ at pressure 70 torr, microwave power $1000 \mathrm{~W}$ and temperature of the sample stage $\sim 450^{\circ} \mathrm{C}$ for 2.5 hours. P doping was performed using the precursor Di-tertiary-butylphosphine. Phosphorous concentration was maintained at $6000-8000 \mathrm{ppm}$ and estimated phosphorous/carbon ratio during growth is $0.002-0.003$. After the growth diamond

films were additionally treated by hydrogen plasma of power $600 \mathrm{~W}$ and at pressure 50 torr. 

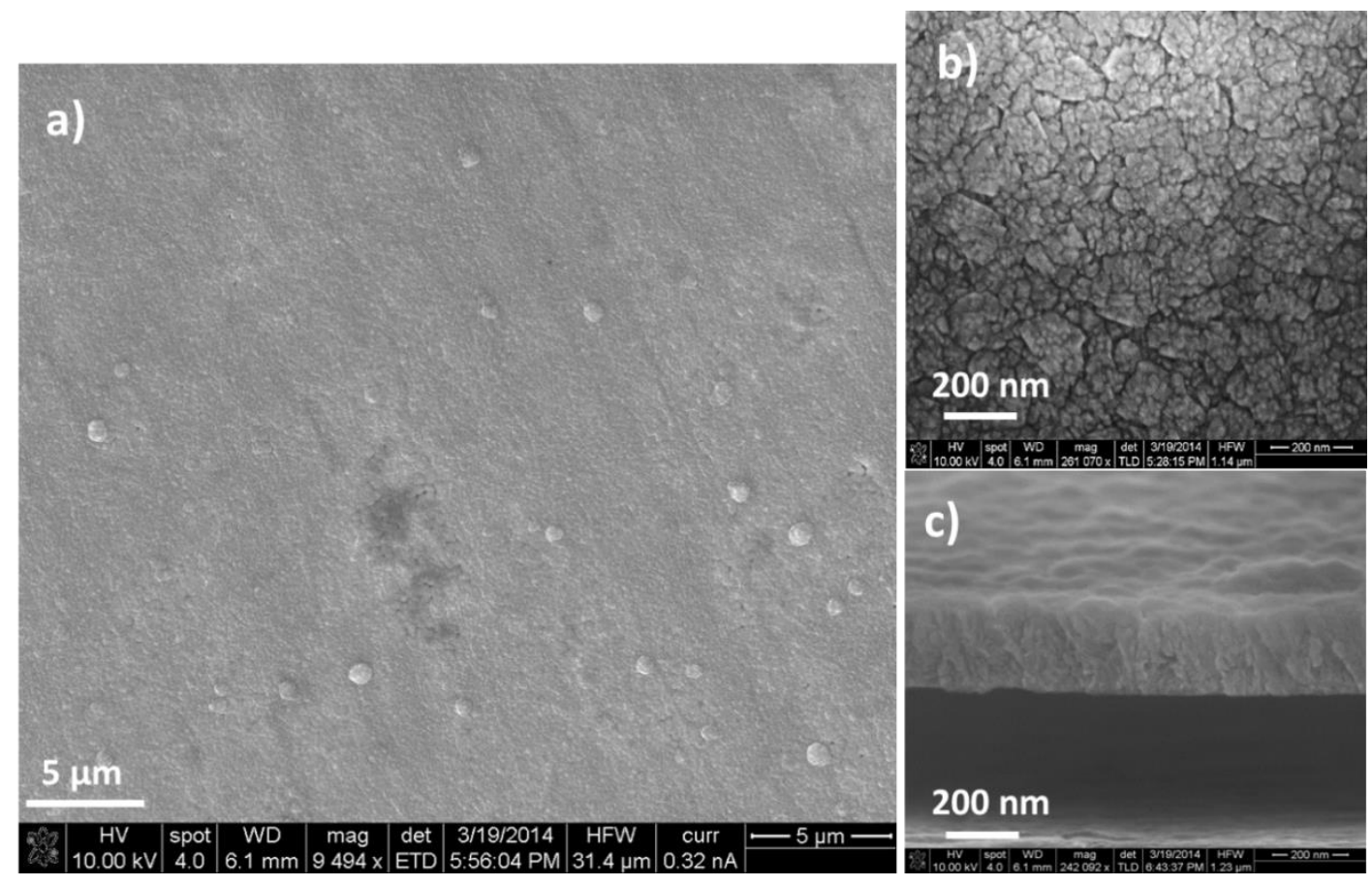

Figure 5.14 SEM images of the $P$ doped nanocrystalline film grown on the $\mathrm{W}$ substrate: a) general low magnification view; b) higher magnification view depicting small size of the grain; c) cross section of the grown diamond film.

(Used with permission - Apendix B).

SEM images (Figure 5.14) show synthesized P doped nanocrystalline diamond

films grown on W substrate. It is evident from the images that the film is uniform (no pinholes) with a less roughness than in the case of $\mathrm{P}$ doped polycrystalline film grown on Si.

Thermionic emission measurements from $\mathrm{P}$ diamond film sample were performed following the same procedure as for the CCNTs coated with diamond. The value of the work function obtained for the $\mathrm{P}$ doped diamond film grown on $\mathrm{W}$ foil is $\sim 1.33 \mathrm{eV}$. The measurements of the second synthesized sample gave value of work function $1 \mathrm{eV}$. These values are closer to the ones reported by Nemanich et al. $0.9 \mathrm{eV}$. 

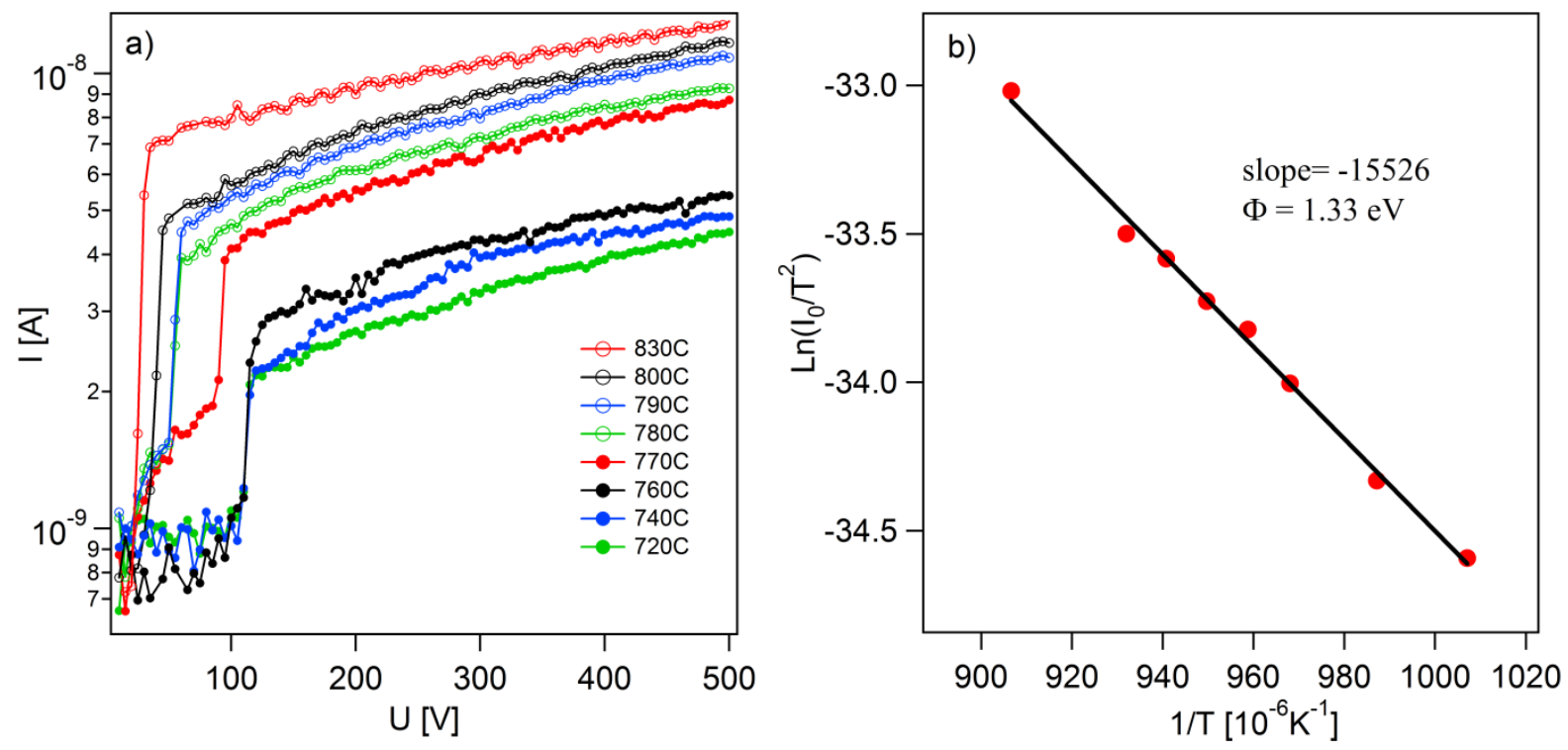

Figure 5.15. Thermionic emission measurements from $P$ doped film grown on $W$ foil: a) thermionic I-V characteristic for different temperatures; b) linear fit of the experimental points - $\operatorname{Ln}\left(\frac{J_{0}}{T^{2}}\right)$ vs $\frac{1}{T}$. (Used with permission - Apendix B).

Even though this time we were capable of performing measurement at lower temperatures $\left(720^{\circ} \mathrm{C}-830^{\circ} \mathrm{C}\right)$, the value of the emission current is very low (Figure 5.14). With maximum value of FETE current $\sim 10 \mathrm{nA}$. Some of the reasons for such low value of the current could be again the Schottky barrier at the $\mathrm{W}-\mathrm{P}$ doped diamond interface as well as inefficient doping by phosphorous.

\subsection{Summary.}

In summary, we presented the work function reduction of phosphorus doped (i) diamond nanocrystals grown on conical carbon nanotubes (CCNTs) and (ii) diamond films grown on silicon substrates. Thermionic emission measurements from phosphorus doped diamond crystals on CCNTs resulted in work function value of $2.23 \mathrm{eV}$. The 
presence of hybrid structure with combination of diamond, CCNTs and graphite, prevented the determination of accurate work function of diamond by UPS. The Ultraviolet Photoelectron spectroscopy Studies on phosphorus doped diamond films yielded a work function value of $1.8 \mathrm{eV}$ with observed NEA value of $1.2 \mathrm{eV}$. Additional thermionic emission measurements of the P doped films grown on $\mathrm{W}$ foil revealed even more reduced value of the work function $1.0-1.3 \mathrm{eV}$. However these samples exhibit very low value of emission current - much lower than in the case of CCNT coated with P doped diamond. 


\section{CHAPTER 6}

\section{TUNGSTEN OXIDE NANOWIRES. THERMIONIC EMISSION PROPERTIES AND WORK FUNCTION}

In this chapter, we report on the thermionic emission properties of hybrid structure of tungsten nanowires decorated with phosphorous doped diamond. The starting material is tungsten oxide nanowires grown on $\mathrm{W}$ foil subsequently reduced to $\mathrm{W} / \mathrm{WOx}$ utilizing hydrogen plasma. Finally, W/WOx nanowires were coated with $\mathrm{P}$ doped nanocrystalline diamond. The thermionic emission results were used to extract the work function values of the W/WOx nanowires and hybrid structure of W/WOx decorated with P doped nanocrystalline diamond. 


\subsection{Synthesis of the Tungsten Oxide nanowires - $\mathrm{W}_{18} \mathrm{O}_{49}$.}

\subsubsection{Introduction.}

As we mentioned in the introduction, in place of CCNT we proposed a hybrid structure consisting of W/WOx nanowires as a conducting channel to support doped diamond nanocrystals. W/WOx nanowires in turn could be obtained by reduction of tungsten oxide structures. Tungsten oxide nanowires have been extensively used in other applications such as gas sensing, photo catalysis etc. Some of the attractive features of these structures are relatively simple synthesis procedure and uniform vertical growth over a large area $[81]\left(\sim 1 \mathrm{~cm}^{2}\right.$ or even more).

\subsubsection{Experimental procedure - synthesis and MW plasma reduction.}

Tungsten oxide nanowires were grown using a custom built hot filament CVD system (Figure 6.1) [81,82]. The experimental setup consists of a quartz tube with the provisions for controlled gas flow and pressure. The W filament (Alfa Aesar $0.5 \mathrm{~mm}$ diameter: length $\sim 3 \mathrm{~m}$ ) is used as the tungsten source and the substrate was heated heater via radiation at high temperatures. Substrates (W foil) are placed underneath the filament on a quartz boat (Figure 6.1). 


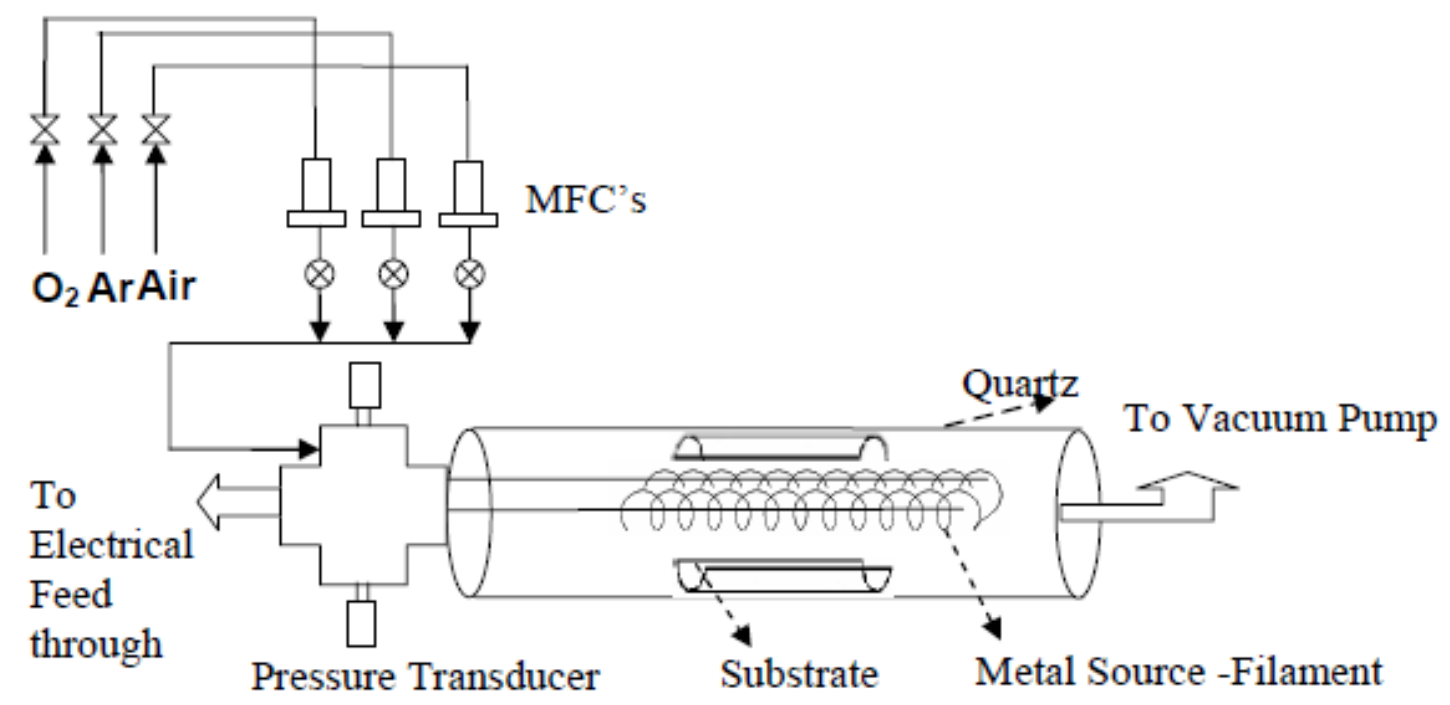

Figure 6.1 Schematic of the Scale up Hot Filament CVD reactor with tungsten filament inside the vacuum chamber (Courtesy of J.Thangala et al. [2]).

The tungsten filament is heated up using an electrical feed-through to a temperature in the range of $1000 \mathrm{~K}-1500 \mathrm{~K}$. The temperatures within the system were monitored using a single wavelength pyrometer (Raytek, Model no. 2838780101). Typically, the substrates (quartz or $\mathrm{W}$ foil) were heated directly by filament to a temperature of about $823 \mathrm{~K}$.

Before the growth, W foils were cleaned in acetone and dried. Growth was performed at the pressure of 380 torr, with an $\mathrm{O}_{2}$ flow of $16 \mathrm{sccm}$. Magnitude of the current through the filament was maintained at $10 \mathrm{~A}$ and $27 \mathrm{~V}$ while the temperature of the filament $\sim 1100 \mathrm{~K}$. Time of the growth was kept for $30 \mathrm{~min}$.

After the growth, reduction of the nanowires from oxide to tungsten was achieved in the MWCVD system AsTeX 5010 (1.5 kW, $2.45 \mathrm{GHz})$. Microwave power was $800 \mathrm{~W}$, the pressure 25 torr, hydrogen flow $200 \mathrm{sccm}$ and the time of the process $40 \mathrm{~min}-1 \mathrm{hr}$. 


\subsubsection{Results $-\mathrm{W}_{18} \mathrm{O}_{49}$ nanowires and after reduction WOx.}

The growth resulted in an array of vertically aligned $\mathrm{W}_{18} \mathrm{O}_{49}$ - confirmed by the XRD measurements (Figure 6.4a) [83]. As it could be seen from the SEM images (Figure

6.2) nanowires are partially aligned - protruding at some angle from the substrate.

a)

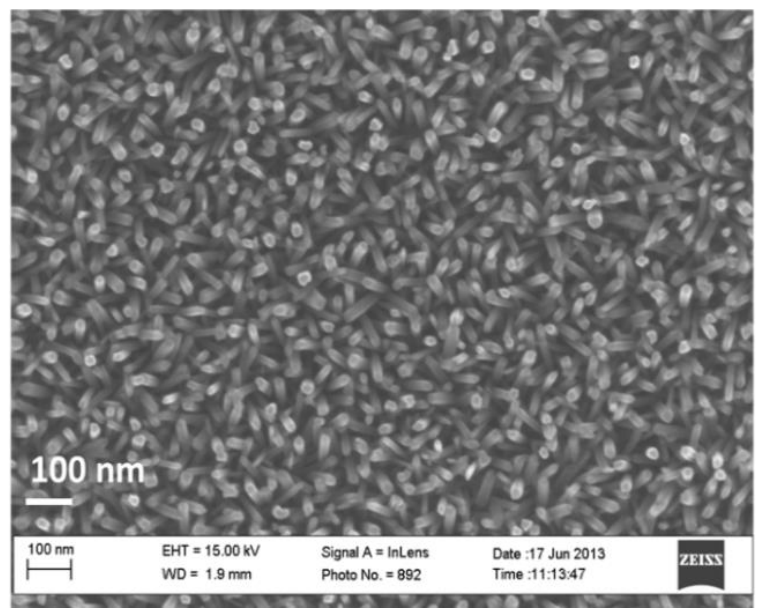

c)

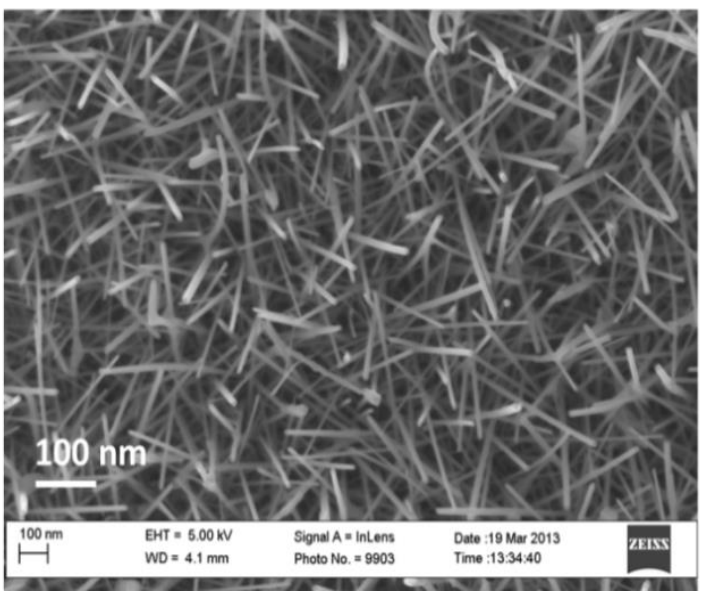

b)

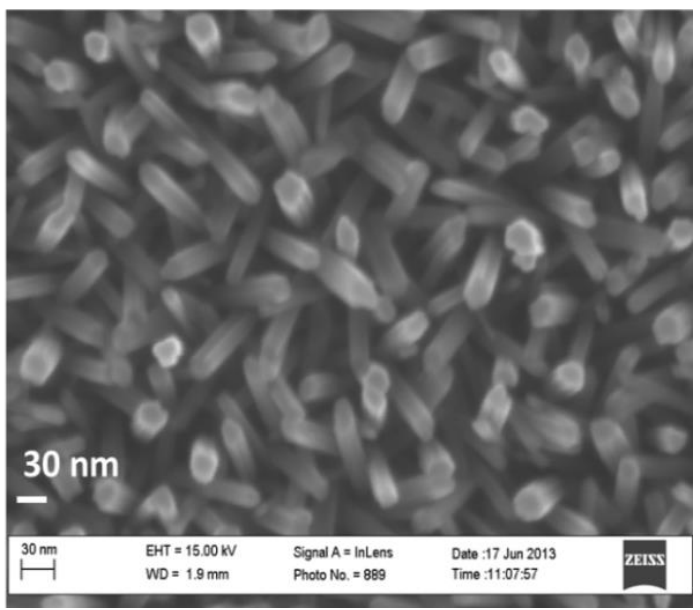

d)

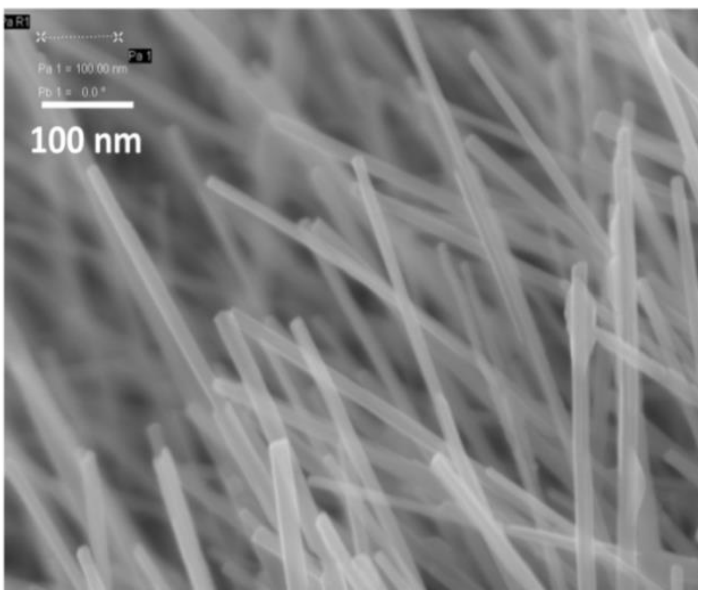

Figure 6.2 Tungsten Oxide nanowires grow non the $\mathrm{W}$ foils: a) sample 1 view $\mathrm{b}$ ) sample 1 at larger magification; c) sample 2 d) larger magnification of sample 2 (tilted SEM image). Clearly seen difference in the morohology particularly diameter of the nanowire. 
It can be clearly seen the difference in the diameters of the nanowires due to the growth conditions. By varying the temperature, growth time, and the pressure it is possible to induce changes in the morphology of the nanowires.

a)

Nanowires before reduction

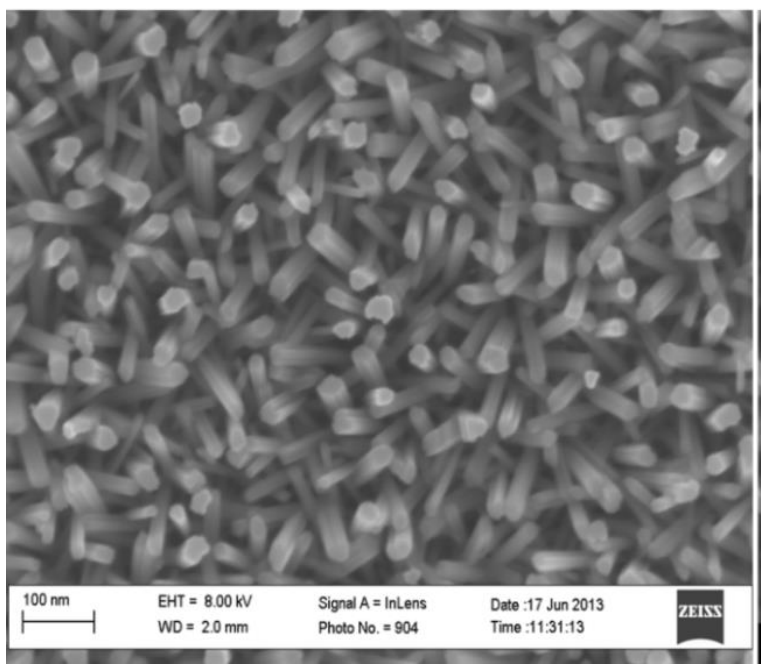

b)

Nanowires before reduction

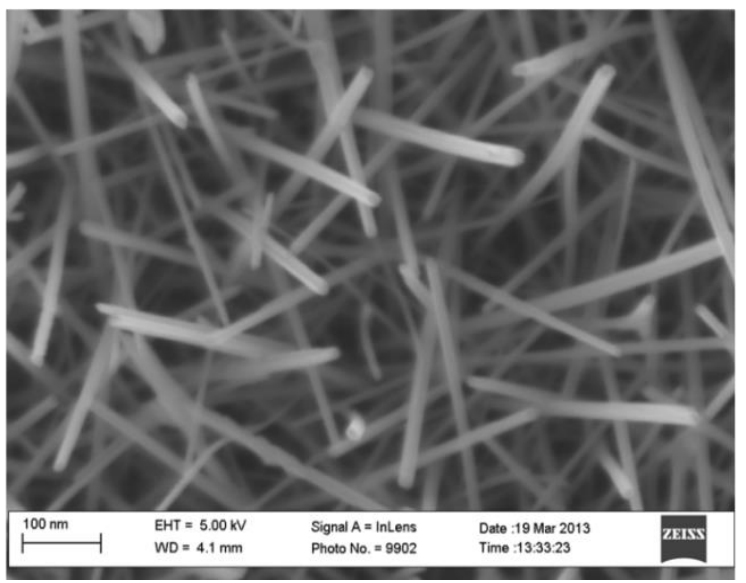

Nanowires after reduction

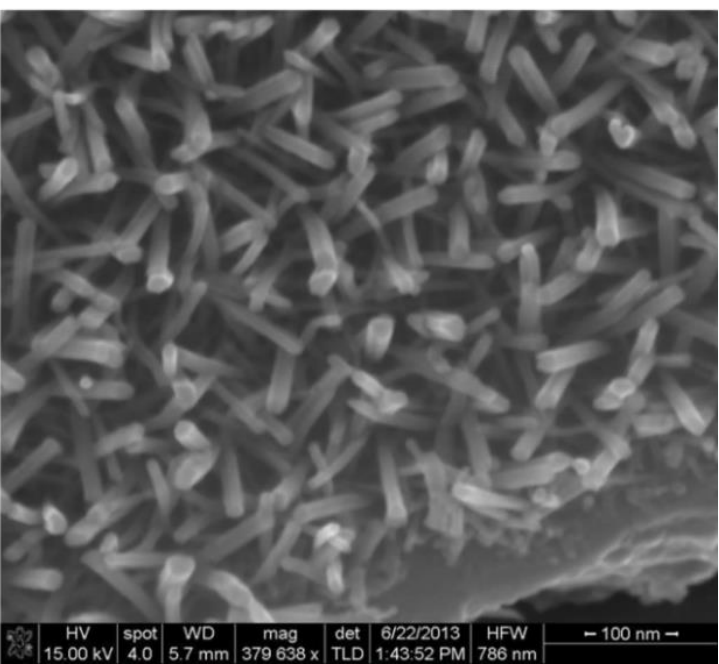

Nanowires after reduction

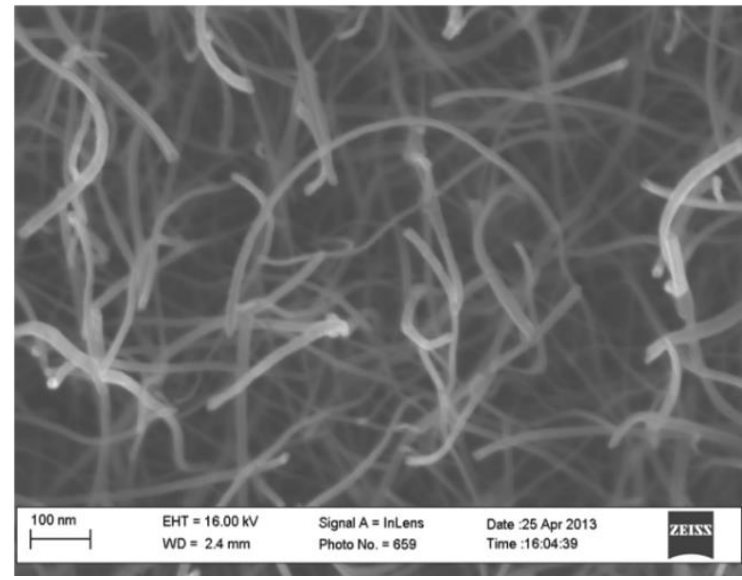

Figure 6.3 a) nanowires of the sample 1 before and fter hydrogen plasma treatment; b) sample 2 before and fter hydrogen plasma treatment 
Average diameter for sample 1 (Figure 6.2ab) $30-40 \mathrm{~nm}$ (length $\sim 100 \mathrm{~nm}$ ) and for the sample 2 (Figure $6.2 \mathrm{~cd}$ ) $15-25 \mathrm{~nm}$ (length $\sim 500 \mathrm{~nm}$ ).

During the proces of reduction using microwave hydrogen plasma, oxygen escapes from the crystal lattice of the nanowire, affecting the structural properties of the nanowire. At least we would expect change of the diameter of the individual nanowire (Figure 6.3 a) due to partial etching by hydrogen plasma or even more drastic changes such as bending of the nanowires (Figure $6.3 \mathrm{~b}$ ).

\subsubsection{Results - $\mathrm{W}_{18} \mathrm{O}_{49}$ nanowires before and after reduction. XRD, RAMAN, TEM.}

In order to confirm the reduction of the tungsten oxide nanowires $\left(\mathrm{W}_{18} \mathrm{O}_{49}\right)$ to tungsten or more oxygen deficient phase $\mathrm{WO}_{\mathrm{x}}$, samples were analyzed using $\mathrm{X}$ ray diffraction (Brucker D8 Advance series 2). Figure 6.4 represents the XRD spectra of the sample 2 before the reduction. They show the characteristic peaks of the $\mathrm{W}_{18} \mathrm{O}_{49}$ (Figure 6.4 a) phase as well strong peaks corresponding to W substrate probably due to exposed edges of the $\mathrm{W}$ foil.

After the hydrogen plasma treatment, the intensity of the characteristic peaks of the $\mathrm{W}_{18} \mathrm{O}_{49}$ dramatically decreased (Figure $6.4 \mathrm{~b}$ ).

Raman analysis of the samples provided unambiguous evidence to support the reduction of the nanowires after hydrogen plasma treatment. Raman measurements were performed using a Renishaw InVia system. A $442 \mathrm{~nm}$ (blue laser, HeCd) excitation wavelength was used for collecting the spectra at $\sim 5 \%$ of the standard power of the laser $(\sim 0.05 \mathrm{~mW})$. Reduction of the power of the laser was necessity in order to avoid oxidation due to heating or burning of the sample. 
a)

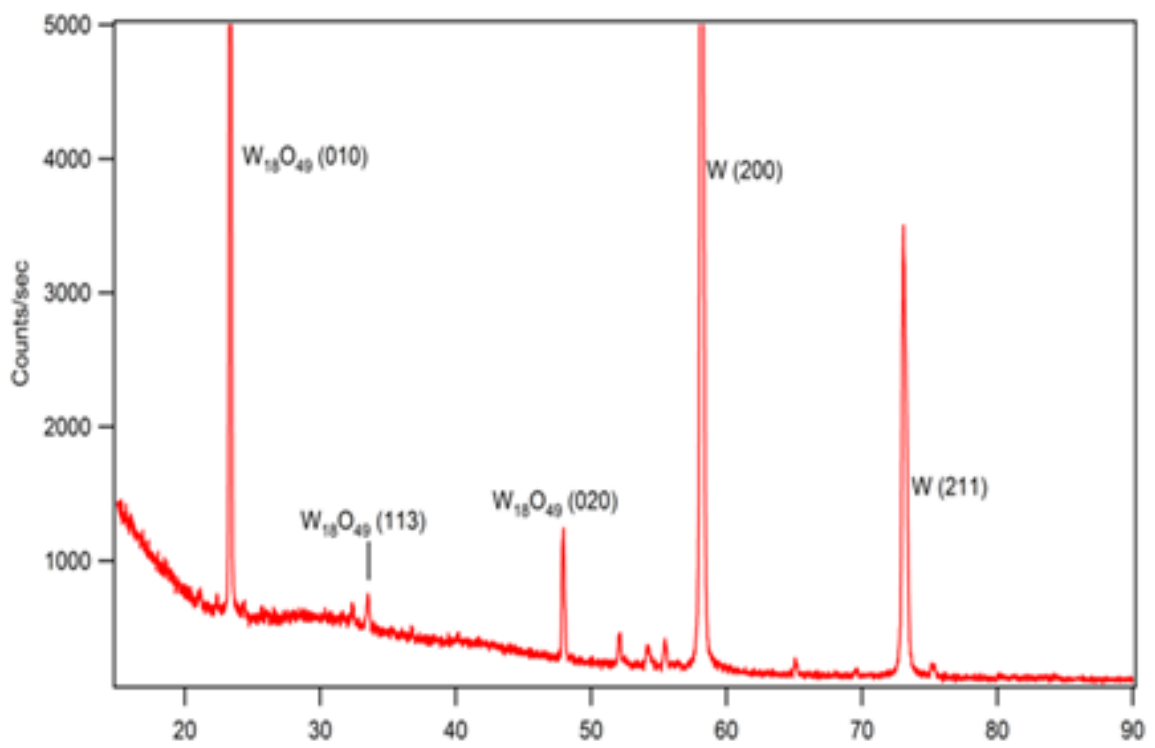

b)

2 Theta

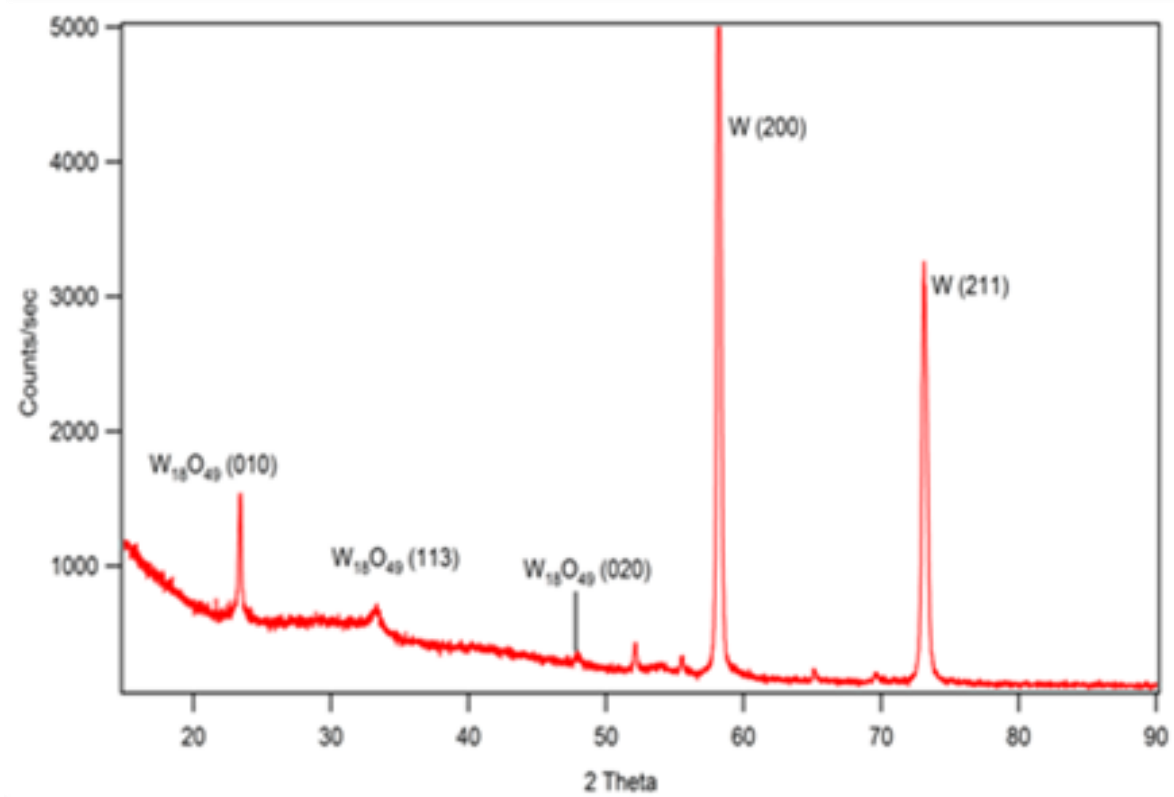

Figure 6.4 XRD spectra of the $\mathrm{W}_{18} \mathrm{O}_{49}$ nanowires (sample 2): a) before hydrogen microwave plasma treatment; b) after the treatment. Most of the diffraction peaks (beside $\mathrm{W}$ peaks) could be indexed to monoclinic $\mathrm{W}_{18} \mathrm{O}_{49}$. [83] 


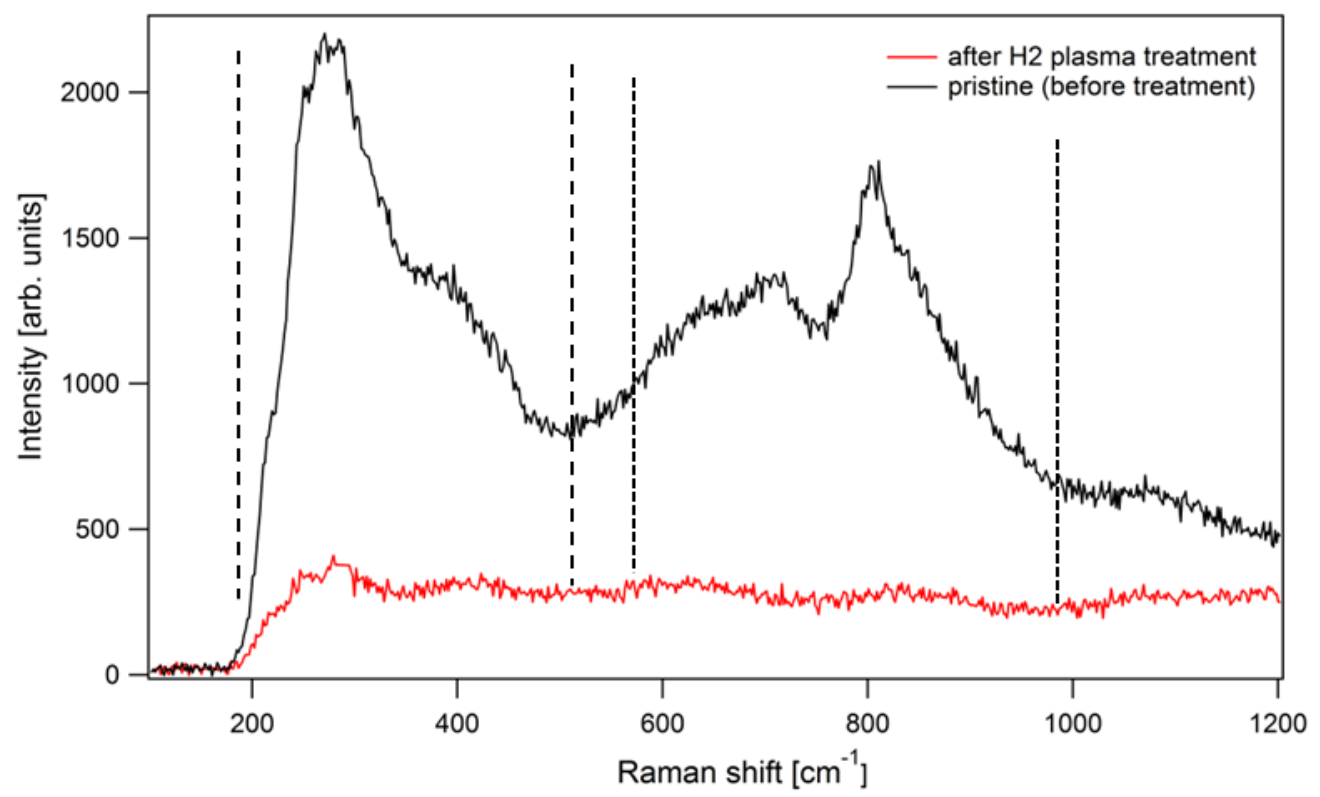

Figure 6.5 Raman spectra of the $\mathrm{W}_{18} \mathrm{O}_{49}$ array sample. By dashed lines are marked ranges of the typical broad bands for the nanowire tungsten oxide samples: 1st: 100$500 \mathrm{~cm}^{-1}$ assgined to $\mathrm{O}-\mathrm{W}-\mathrm{O}$ bending modes; $2 \mathrm{nd}: 600-1000 \mathrm{~cm}^{-1}$ contributed by $\mathrm{W}-$ O stretching modes $[84,85]$.

From the Raman spectra presented in Figure 6.5 it is clearly seen the disappearance of the characteristic broad bands for the tungsten oxide nanocrystalline structures $[84,85]$ implying effective reduction of the oxide.

Finally, analysis by the Transmission Electron Microscopy (TEM) including SAED and EDAX revealed some interesting details of the individual nanowires.

From the TEM images it is clear that each individual $\mathrm{W}_{18} \mathrm{O}_{49}$ nanowire (before hydrogen plasma treatment) is single crystalline. The crystalline faces are clearly visible in the image (Figure $6.6 \mathrm{c}$ ). 
Before the reduction
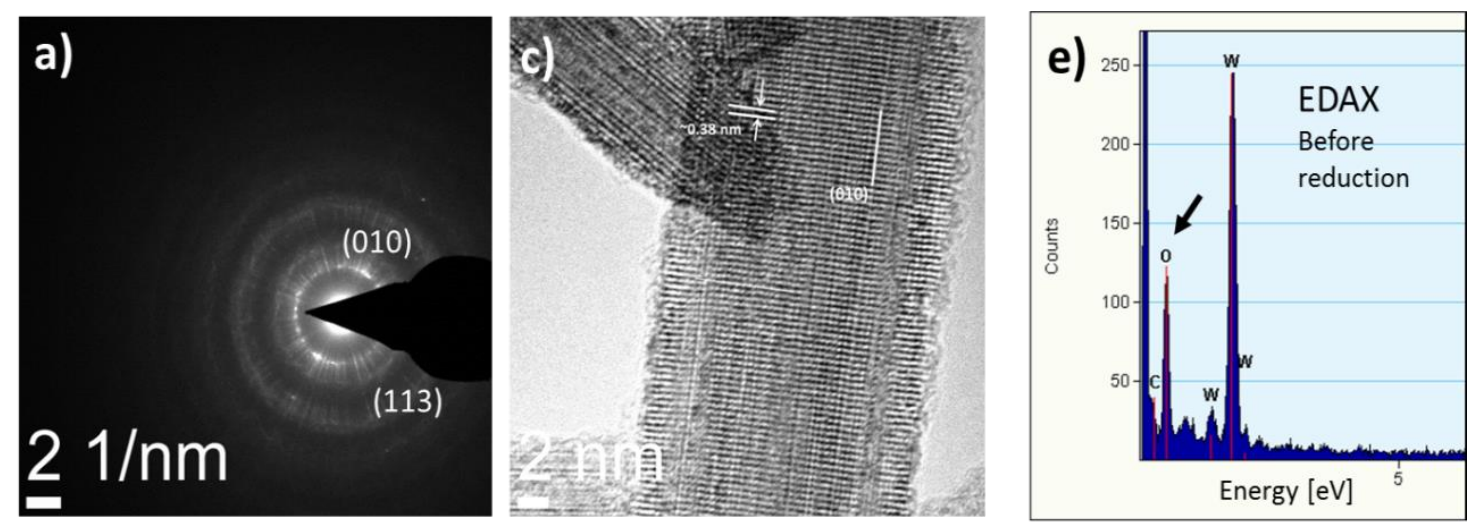

After the reduction
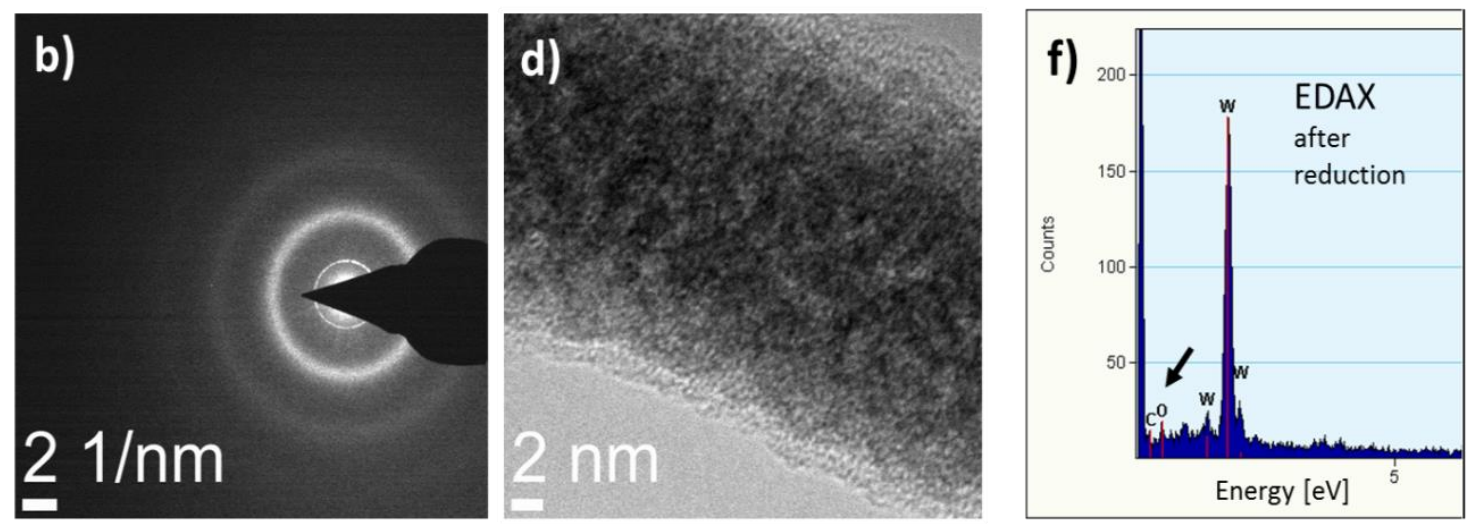

Figure 6.6 Selected Area Electron Difraction images of the $\mathrm{W}_{18} \mathrm{O}_{49}$ individual nanowire a) before recution and $b$ ) after; TEM images $-c$ ) befor reduction and d) after. EDAX spectra e) before and after f).

Selected Area Electron Diffraction (SAED) of individual nanowires supports this hypothesis with the presence of a pattern of dots (Figure 6.6 a) and we can assign an appropriate crystalline face to the pattern with respect to the XRD data (Figure $6.5 \mathrm{a}$ ). However, in the case of reduced nanowires the SAED pattern consists of concentric rings (Figure $6.6 \mathrm{~b}$ ) which is characteristic for polycrystalline or amorphous materials. Moreover, the TEM image of the reduced nanowire does not show any clear faces of the crystal (Figure $6.5 \mathrm{~d}$ ). It seems that after hydrogen plasma treatment, nanowire has an 
amorphous (porous) structure or ultra nanocrystlline structure where we cannot clearly see the grains. In addition EDAX spectra from the nanowire (Figure $6.6 \mathrm{e}, \mathrm{f}$ ) show clear reduction of the oxygen peak after the hydrogen plasma treatment (marked by an arrow in the graph).

\subsection{Synthesis of the $P$ doped diamond on the reduced W/WOx nanowires.}

P doped crystals were grown on the reduced array of WOx nanowires (sample 2). Before the growth, samples were pretreated as usual by sonication in colloidal solution of the ethanol and diamond powder (1-10 nm). However time of treatment of the WOx samples was significantly reduced. W foils with the array of WOx nanowires was dipped in to the solution for a short time $(15 \mathrm{sec})$ in a sonication bath. This procedure was repeated while sonicating and finally cleaned in pure ethanol.

After that samples were loaded into the chamber of the Astex 5010 MWCVD system. Depositions were performed at slightly different conditions in comparison to the growth of doped diamond on CCNT. Microwave power: $900 \mathrm{~W}$, pressures: 50 torr with $2.5 \%$ methane in hydrogen with total flow rate of $200 \mathrm{sccm}$. The phosphorus concentration was set for 10,000 ppm (with vapor pressure of Di-TBP being measured as 1.7 torr). Time of the growth was maintained for 1 hour.

As a result of the growth califlower like diamond structures (Figure 6.7) were obtained on top of the nanowires. 

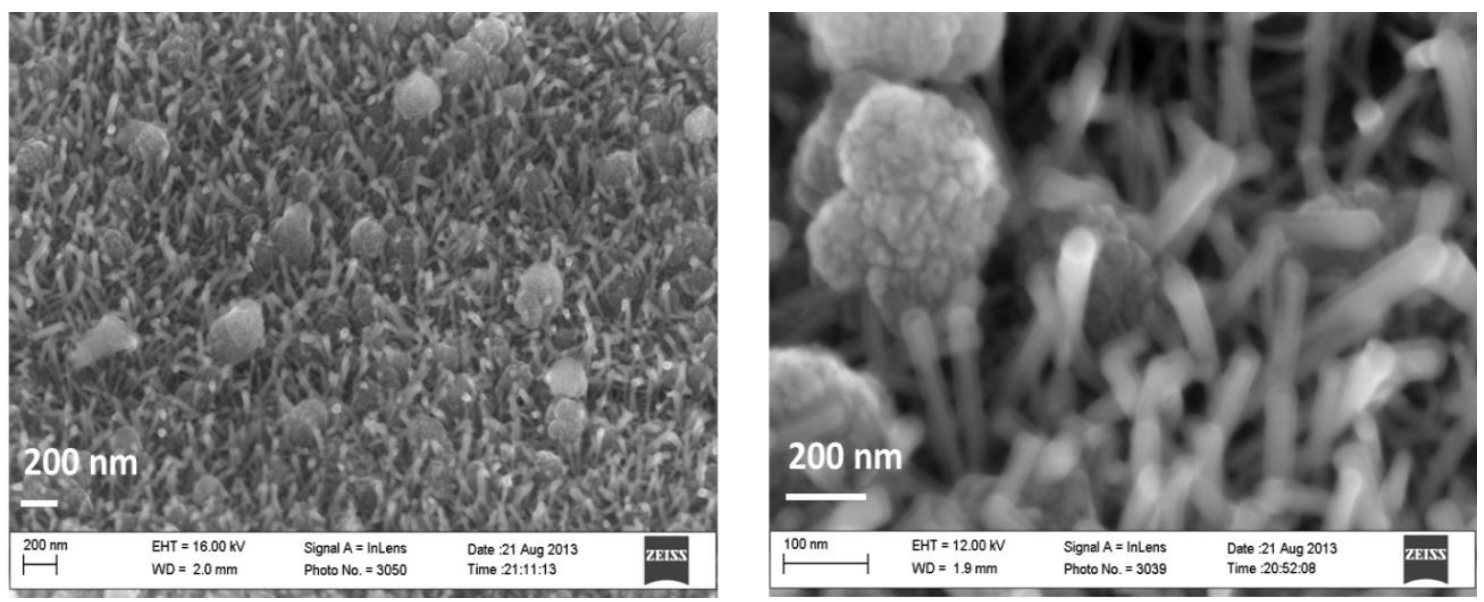

Figure 6.7 SEM images of the P doped diamond nanocrystallien aglomerations grown on the reduced WOx nanowires.

Given the comparably short lengths of the nanowires $(\sim 100 \mathrm{~nm})$, some of the diamond agglomerations completely coated groups of nanowires. It is hard to determine whether any of the diamond crystals or nanocrystalline structures was coated only at the tip of the individual nanowire. Nucleation of the diamond during the growth could have been initiated at the substrate among the nanowires.

\subsection{Thermionic emission measurements of the WOx nanowires coated by $\mathbf{P}$ doped diamond.}

Thermionic emission measurements of the WOx nanowire sample with $\mathrm{P}$ doped diamond (sample 2) was performed using the same experimental system as for other samples described in the Chapter 3. Measurement procedure was the same as described in Chapter $2-4$. First we determined value of the work function of reduced WOx nanowires sample to be $\sim 5.2 \mathrm{eV}$ comparable for polycrystalline $\mathrm{W}$. However, there have 
been reports on the work function values determined by TE in the range $5.2-5.33 \mathrm{eV}$ for some faces of the single crystalline $\mathrm{W}-(110),(011,(112))$.

a)

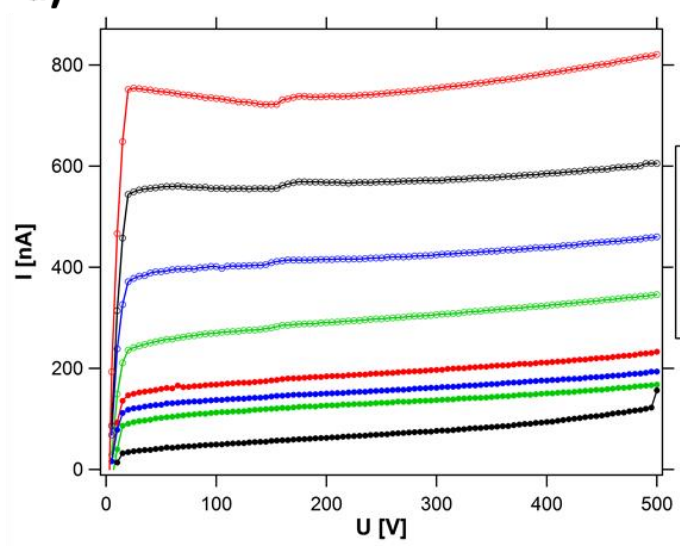

b)

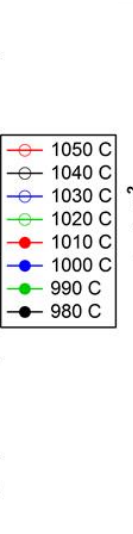

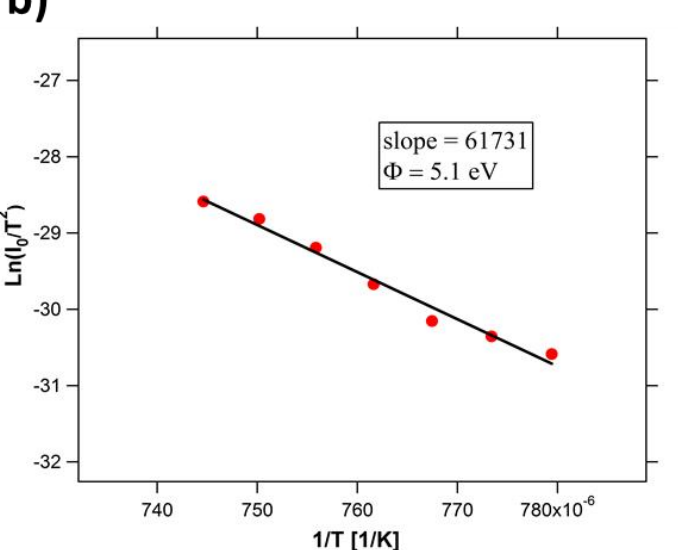

Figure 6.8 a) Emission current versus applied voltage at different temperatures for WOx nanowire coated with P doped. b) Richardson-Dushman linear fitting.

Value of the work function determined for P-doped diamond supported on WOx nanowires by thermionic emission measurements is unexpectedly higher than that of $\mathrm{P}$ doped diamond on CCNT (2.23 eV). The reason for such higher value could be explained based on similar mechanisms in case of the diamond coated CCNTs and P doped diamond films the band diagram illustrating possible two scenarios are presented in figure 6.9. Also we have to note that considering minimum temperature at which we were able to measure emission current was $980{ }^{\circ} \mathrm{C}$, it is reasonable to expect that diamond surface is not terminated by hydrogen and studied $\mathrm{P}$ doped diamond has positive electron affinity. 


\section{P doped diamond on W/WOx nw}

a)

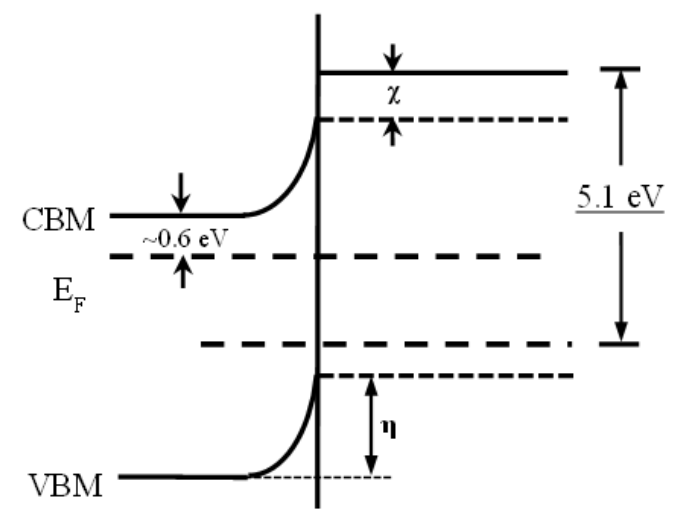

$\mathrm{E}_{\mathrm{F}}-\mathrm{MBG}=4.5-\eta-\chi$ b)

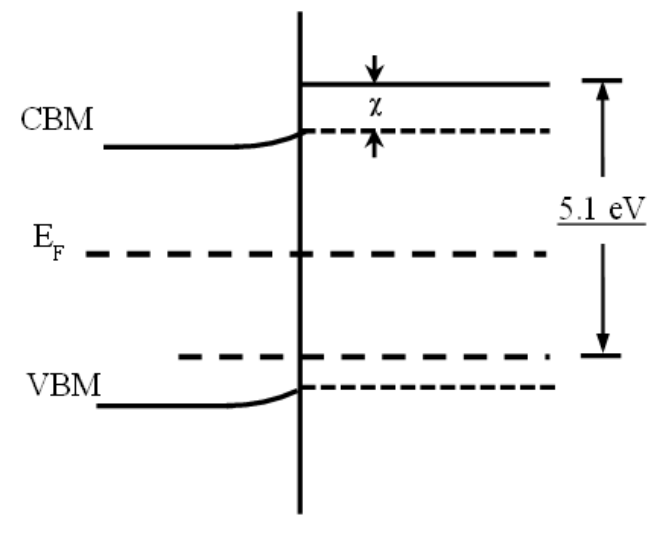

$\mathrm{MBG}-\mathrm{VBM}=5.5-(5.1-\chi)$

MBG $-\mathrm{VBM}=0.4+\chi$

Figure 6.9 Surface energy band diagram of $\mathrm{P}$ doped nanocrystals grown on the $\mathrm{W} / \mathrm{WO}_{\mathrm{x}}$ nanowires.

In the first case (Figure 6.9a), due to presence of the surface defect states and assumed $\mathrm{P}$ donor level to be at $0.6 \mathrm{eV}$ below $\mathrm{CBM}$, there is present upward band bending at the surface. Additionally, considering that diamond structure grown on nanowires is nanocrystalline it is possible that there are present mid-band gap states. MBG position should be much lower in the band gap close to valence band maximum compared to earlier discussed P doped diamond crystals on CCNTs and P doped diamond film, what is implied by the high value of the measured work function $5.1 \mathrm{eV}$. The value of the band bending $\eta$ is expected to be not very large (less than in case of first hybrid structure and $\mathrm{P}$ doped diamond film) because of the weak contribution of the electrons from the $\mathrm{P}$ donor level. Otherwise, the lower value of the work function would have been expected because of the effective contribution of the significant population of electrons by the $\mathrm{P}$ impurities in diamond. Which would suggest that during emission electrons were mostly excited from the Fermi level of the P doped diamond - $0.6 \mathrm{eV}$ below CBM. 
In the second scenario (Figure 6.9b), The Fermi level is much lower in the band gap and there is weak upward band bending. In sum, a large value of the work function is related to the low position of the MBG placed much closer to the VBM compared to the first case (Figure 6.9a).

\subsection{Summary}

Combined characterization techniques (XRD, Raman, TEM) showed efficient reduction of the tungsten oxide nanowires due to hydrogen plasma treatment. However, removal of the oxygen from crystal lattice creates the porous structure: made up mostly of $\mathrm{W}$ atoms of ultra nanocrystalline or even amorphous. The deduced structure is expected to have electronic properties very different from the single crystal monoclinic $\mathrm{W}_{18} \mathrm{O}_{4}$ leading to poor emission properties as evidenced by the high values of the work function $(\sim 5.1 \mathrm{eV})$ of the hybrid structure consisting of reduced WOx nanowires and $\mathrm{P}$ doped diamond. 


\section{CHAPTER 7}

\section{CONCLUSIONS}

In this work we studied novel hybrid structures of CCNT coated with P doped diamond. The main goal was to study the thermionic emission properties to determine the work function of $\mathrm{P}$ doped diamond. We also proposed an alternative material in place of CCNT, W/WOx nanowires. Considering its specific properties, W was picked as the material of choice due to its use in field emission and thermionic emission applications. As a part of the study of the thermionic emission properties of the hybrid structures, first we concentrated on the pristine CCNT, as they play an important role as the conducting backbone for the P doped diamond.

CCNT samples were synthesized on Pt wires and graphite foils with different densities, tips sizes, lengths, and wall structures. The work function $\phi$ was determined by thermionic emission, while the range of $\phi$ values for each sample could be indirectly related to the morphological characteristics such as aspect ratio, density, and wall structure of CCNTs. The observed lower values for $\phi$ are significantly smaller than that reported for multi-walled carbon nanotubes (MWNTs). The reduced $\phi$ values could be attributed to the local electric field enhancement of the CCNTs and as a consequence, field penetration effect that is capable of significantly reducing potential barrier. The UPS 
measurements of CCNTs grown on Pt wires show higher values of work function compared to the values obtained by thermionic emission method, thus supporting our assumption regarding field penetration effect.

In the next part of this work we studied the emission properties of the hybrid structure. For better understand of the underlying mechanism of the emission properties of our structure we further studied ultraviolet photoelectron emission from the $\mathrm{P}$ doped diamond films. Ultimately, the work function reduction of phosphorus doped (i) diamond nanocrystals grown on conical carbon nanotubes (CCNTs) and (ii) diamond films grown on silicon substrates are presented. The CCNTs provide the conducting backbone for the P-doped diamond microcrystals as well allow to increase the surface area of the former. Thermionic emission measurements from phosphorus doped diamond crystals on CCNTs resulted in work function value of $2.23 \mathrm{eV}$. The presence of hybrid structure with combination of diamond, CCNTs and graphite, prevented the determination of accurate work function of diamond by UPS. The Ultraviolet Photoelectron spectroscopy studies on phosphorus doped diamond films yielded a work function value of $1.8 \mathrm{eV}$ with observed NEA value of $1.2 \mathrm{eV}$. The origin of the reduced values of work functions is discussed using detailed band diagrams. Proposed explanation of work function reduction for the doped diamond crystals supported on CCNTs is upward band bending due to P doping and difference between bulk and surface Fermi levels. We suggest that midband-gap state due to the structural defects of the polycrystalline diamond could be an additional factor affecting value of the work function. Finally, as a third possibility only for $\mathrm{P}$ doped diamond coated CCNTs and P doped diamond films, it could be considered that there is a significantly lower position of Fermi level than expected for this type of doping. For P 
doped diamond films, the further decrease of work function value is attributed to negative electron affinity.

Thermionic emission results from the $\mathrm{P}$ doped diamond films grown on $\mathrm{W}$ foil revealed that these structures have lower value of work function in the range $1.0-1.33$ $\mathrm{eV}$ in agreement with the value reported by Nemanich et al.. However, these films show very low emission currents.

In order to realize the idea of an alternative hybrid structure, vertically grown Tungsten oxide $\left(\mathrm{W}_{18} \mathrm{O}_{49}\right)$ nanowires on $\mathrm{W}$ foil were studied. It was revealed that reduction of $\mathrm{W}_{18} \mathrm{O}_{49}$ nanowires by hydrogen plasma removes oxygen from crystalline lattice of the nanowires and as a result the reduced W/WOx nanowires have a porous structure.

Thermionic emission measurements from the alternative hybrid structure of W/WOx nanowires coated with $\mathrm{P}$ doped diamond showed a high work function value exceeding $5.0 \mathrm{eV}$. Suggested explanation of the large value of the $\phi$ is weak contribution from the P donor levels and low position of the mid-band gap states compared to CCNTs coated with P doped diamond and P doped diamond film. There is no clear explanation for such position of the MBG states and negligible role of the P doping in case of the alternative hybrid structure and this issue requires further investigation. 


\section{REFERENCES}

[1] Reimann, A. L., Thermionic Emission. John Wiley and Sons, Inc., New York (1934)

[2] Hatsopoulos, G. N.; Gyftopoulos, E. P., Thermionic Energy Conversion I. Cambridge, MA: MIT Press. ISBN 0-262-08059-1 (1974)

[3] Smith, J. R.; Bilbro, G. L. and Nemanich, R. J., Theory of space charge limited regime of thermionic energy converter with negative electron affinity emitter, J. Vac. Sci. Technol. B 2 (2009) 1132

[4] Field, J. E., The properties of diamond. Academic Press (1979)

[5] Koeck, F. A. M.; Nemanich, R. J.; Lazea, A.; Haenen, K., Thermionic electron emission from low work-function phosphorus doped diamond films, Diamond and Related Materials 18 (5 - 8) (2009) 789-791

[6] Andriy Sherehiy, Santoshrupa Dumpala, Mahendra.K. Sunkara, Jacek B. Jasinski, Robert W.Cohn and Gamini U. Sumanasekera, Thermionic emission from phosphorus (P) doped diamond nanocrystals supported by conical carbon nanotubes and ultraviolet photoelectron spectroscopy study of P-doped diamond films, Diamond and Related Materials, In press, DOI: 10.1016/j.diamond.2014.09.005 (2014) 
[7] Santoshrupa Dumpala, Nanoscale diamond and carbon materials and architecture for field emission and thermionic energy conversion, Phd dissertation, (2011)

[8] Kittel, Introduction to Solid State Physics, (1995)

[9] A.Modinos Field, Thermionic, and Secondary Electron Emission Spectroscopy, Plenum Press, NYC, (1984)

[10] Landau and Lifshitz, Quantum Mechanics, (1965)

[11] E.L. Murphy and R.H.Good, Thermionic emission, field emission, and transition region, Phys. Rev., 102, 1464, (1956)

[12] Richard G Forbes and Jonathan H.B Deane, Reformulation of the standard theory of Fowler-Nordheim tunneling and cold field electron emission, Proc. R. Soc. A 2007 463, doi: 10.1098/rspa.2007.0030, (2007)

[13] C.J.Edgecomb and U.Valdre, Philosophical Magazine Part B 82, 987 (2002)

[14] R.G.Forbes, C.J.Edgecombe, and U.Valdre, Ultramicroscopy 95, 57 (2002); G.Fursey, Field Emission in vacuum microelectronics (Kluwer Academic and Plenum Publihers), New York, (2003)

[15] M.Bagge-Hansen, R.A.Outlaw, P.Mirado, M.Y.Zhu, K.Hou, N.D. Theodore, X.Zhao and D.M.Manos, Journal of Aplied Physics 103, 014311 (2008)

[16] J.C. Riviere, Work function: Measurements and results, Solid State Surface Science, Vol 1, New York:Marcel Dekker, (1969)

[17] A.G. Rinzler, J.H. Hafner, P. Nikolaev, L. Lou, S.G. Kim, D. Tomanek, D. Colbert, R.E. Smalley, Science 269 (1995) 1550-1553.

[18] W.A.D. Heer, A. Chatelain, D. Ugarte, Science 270 (1995) 1179-1180.

[19] J.M. Bonard, J.P. Salvetat, T. Stöckli, W.A. de Heer, L. Forró, A. Châtelain, Appl. Phys. Lett. 73 (1998) 918.

Q.H. Wang, T.D. Corrigan, J.Y. Dai, R.P.H. Chang, A.R. Krauss, Appl. Phys. Lett. 70 (1997) 3308. 
M. Sveningsson, R.E. Morjan, O.A. Nerushev, Y. Sato, J. Backstrom, E.E.B. Campbell, F. Rohmund, Appl. Phys., A Mater. Sci. Process. 73 (2001) 409

[20] Jijun Zhao, Jie Han, and Jian Ping Lu, Phys Rev B, 65 (2002) 193401.

[21] Bin Shan, Kyeongjae Cho, Phys. Rev. Lett. 94 (2005) 236602.

[22] Xiao Zheng, GuanHua Chen, Zhibing Li, Shaozhi Deng, and Ningsheng Xu, Phys. Rev. Lett. 92 (2004) 106803.

[23] Zhou, G.; Duan, W.; Gu, B. Phys. Rev. Lett. 87 (2001), 095504

[24] C.-W. Chen, M.-H. Lee, S.J. Clark, Appl. Surf. Sci. 228 (2004) 143

A. Mayer, N.M. Miskovsky, P.H. Cutler, Phys. Rev. B 65 (2002) 155420

[25] Masashi Shiraishi, Masafumi Ata, Carbon, 39 (2001) 1913.

[26] S. Suzuki, Y. Watanabe, Y. Homma, S. ya Fukuba, S. Heun, and A. Locatelli, Appl. Phys. Lett. 85 (2004) 127.

[27] H. Ago, Th. KugIer, F. Caciaili, K. Petritsch, R.H. Friend, W.R. Salaneck, Y. Ono, T. Yamabe, and K. Tanaka, Synthetic Metals, 103 (1999) 2494.

[28] S. Suzuki, C. Bower, Y. Watanabe, O. Zhou, Appl. Phys. Lett., 76 (26) (2000) 4007.

[29] R. Gao, Z. Pan, and Z. L. Wang, Appl. Phys. Lett. 78 (2001) 1757.

[30] X. Cui, M. Freitag, R. Martel, L. Brus, and P. Avouris, Nano Lett. 3 (2003) 783.

[31] D.C. Cox, R.D. Forrest, P.R. Smith, S.R.P. Silva, Appl. Phys. Lett. 85 (2004) 2065.

[32] Peng Liu, Qin Sun, Feng Zhu, Kai Liu, Kaili Jiang, Liang Liu, Qunqing Li, and Shoushan Fan, Nanoletters Vol. 8, No. 2 (2008) 647-651.

[33] Peng Liu, Yang Wei, Kaili Jiang, Qin Sun, Xiaobo Zhang, Shoushan Fan, Shufeng Zhang, Chuangang Ning, and Jingkang Deng, Physical Review B 73 (2006) 235412.

[34] U.V. Azizov, G.N.Schuppe, Sov. Phys. Solid State, 7 (1966) 1591 - 1595

[35] Feng Jin, Yan Liu and Christopher M. Day, Appl. Phys. Lett., 88 (2006) 163116.

[36] Yang Wei, Kaili Jiang, Xiaofeng Feng, Peng Liu, Liang Liu, and Shoushan Fan Phys Rev B 76, (2007) 045423 
[37] S.K. Kolekar, S.P. Patole, P.S. Alegaonkar, J.B. Yoo, C.V. Dharmadhikari Applied Surface Science 257 (2011) 10306- 10310

[38] R.P. Raffaelle, B.J. Landi, J.D. Harris, S.G. Bailey, A.F. Hepp, Materials Science and Engineering B 116 (2005) 233-243.

[39] Geoffrey A. Landis, Isay L. Krainsky, Sheila G. Bailey, Jeffrey M. Elich, Brian J. Landi, Thomas Gennett, and Ryne P. Raffaelle, NASA Glenn Research Center (2004), http://www.grc.nasa.gov/WWW/RT/2003/5000/5410landis2.html (2012).

[40] Field, J. E. The properties of diamond. Academic Press: 1979.

[41] Smith, J. R.; Bilbro, G. L. and Nemanich, R. J., Theory of space charge limited regime of thermionic energy converter with negative electron affinity emitter, J. Vac. Sci. Technol. B 2 (2009) 1132.

[42] F. Maier, J. Ristein, and L. Ley, Electron affinity of plasma-hydrogenated and chemically oxidized diamond (100) surfaces, Phys. Rev. B 64 (2001) 165411.

J. Ristein, Diamond surfaces: familiar and amazing, Appl. Phys. A 82, (2006) $377-384$

Ristein, J.; Maier, F.; Riedel, M.; Cui, J.B. and Ley, L., Surface Electronic Properties of Diamond, Phys. Stat. Sol. (a) 181 (2000) 65.

[43] J. B. Cui, J. Ristein, and L. Ley, Electron Affinity of the Bare and Hydrogen Covered Single Crystal Diamond (111) Surface, Phys. Rev. Lett. 81 (1998) 429; L. Diederich, P. Aebi, O. M. Kuttel, and L. Schlapbach, NEA peak of the differently terminated and oriented diamond surfaces, Surf. Sci. 424 (1999) L314; P. K. Baumann and R. J. Nemanich, Characterization of copper-diamond (100), (111), and (110) interfaces: 
Electron affinity and Schottky barrier, Phys. Rev. B 58 (1998) 1643; Y.M. Wang, K.W. Wong, S.T. Lee, M. Nishitani-Gamo, I. Sakaguchi, K.P. Loh, and T. Ando, Recent studies on diamond surfaces, Diamond Relat. Mater. 9 (2000) 1582.

[44] Kraft, A., Doped Diamond: A Compact Review on a New, Versatile Electrode Material, Int. J. Electrochem. Sci. 2 (5) (2007) 355-385.

[45] Mainwood, A., Theoretical modelling of dopants in diamond, J. Mater. Sci.Mater. Electron. 17(6) (2006) 453-458.

[46] Farrer, R. G., On the substitutional nitrogen donor in diamond, Solid State Communications 7(9) (1969) 685-688; P. Zapol, M. Sternberg, L. A. Curtiss, T. Frauenheim, D. M. Gruen, Tight-binding molecular-dynamics simulation of impurities in ultrananocrystalline diamond grain boundaries, Phys. Rev. B 65 (2001) 045403.

[47] Eaton, S. C.; Anderson, A. B.; Angus, J. C.; Evstefeeva, Y. E.; Pleskov, Y. V., Co-doping of Diamond with Boron and Sulfur, Electrochem. Solid State Lett 5(8) (2002) G65-G68.

[48] Hasegawa, M.; Teraji, T.; Koizumi, S., Lattice location of phosphorus in $n$-type homoepitaxial diamond films grown by chemical-vapor deposition, Appl. Phys. Lett. 79(19) (2001) $3068-3070$.

Pinault, M.-A.; Barjon, J.; Kociniewski, T.; Jomard, F.; Chevallier, J., The n-type doping of diamond: Present status and pending questions, Physica B 401-402 (2007) 51-56.

[49] Kuriyama, K.; Kimura, C.; Koizumi, S.; Kamo, M.; Sugino, T., Elucidation of field emission characteristics of phosphorous-doped diamond films, J. Vac. Sci. Technol. B 17(2) (1999) 723-727. 
[50] C Chen, C. F.; Tsai, C. L.; Lin, C. L., Electronic properties of phosphorus-doped triode-type diamond field emission arrays, Mater. Chem. Phys. 72(2) (2001) 210213.

[51] H. Kato, S. Yamasaki, H. Okushi, n-type conductivity of phosphorus-doped homoepitaxial single crystal diamond on (001) substrate, Diamond and Related Materials 14(11-12) (2005) 2007-2010.

[52] Takeuchi, D.; Kato, H.; Ri, G. S.; Yamada, T.; Vinod, P. R.; Hwang, D.; Nebel, C. E.; Okushi, H.; Yamasaki, S., Direct observation of negative electron affinity in hydrogen-terminated diamond surfaces, Appl. Phys. Lett. 86 (2005) 152103.

[53] Kono, S.; Mimochi, K.; Takyo, G.; Goto, T.; Abukawa, T.; Aoyama, T., Energy band diagram of a H-terminated P-doped n-type diamond (111) surface, New Diam. Front. Carbon Technol. 17(5) (2007) 231-242.

[54] Lazea, A.; Barjon, J.; D’Haen, J.; Mortet, V.; D’Olieslaeger, M.; and Haenen, K., Phosphorus incorporation in plasma deposited diamond films, J. Appl. Phys., 105 (2009) 083545.

[55] Ghodbane, S.; Omnes, F.; Bustarret, E.; Tavares, C.; Jomard, F., n-type phosphorus-doped polycrystalline diamond on silicon substrates, Diamond and Related Materials 17 (7-10) (2008) 1324-1329.

[56] Kato, H.; Yamasaki, S. and Okushi, H., Growth and characterization of phosphorus-doped diamond using organophosphorus gases, Phys. Stat. Sol. A Applications and Materials Science 202 (11) (2005) 2122-2128.

[57 ] Kataoka, M.; Zhu, C. Y.; Koeck, F. A. M.; Nemanich, R. J., Thermionic electron emission from nitrogen-doped homoepitaxial diamond, Diamond and Related Materials 19 (2-3) (2010) 110-113. 
[58] Suzuki, M.; Ono, T.; Sakuma, N.; Sakai, T., Low-temperature thermionic emission from nitrogen-doped nanocrystalline diamond films on n-type Si grown by MPCVD,

Diamond and Related Materials 18 (10) (2009) 1274-1277.

[59] Koeck, F. A. M.; Nemanich, R. J., Low temperature onset for thermionic emitters based on nitrogen incorporated UNCD films,

Diamond and Related Materials 18 (2-3) (2009) 232-234.

[60] Uppireddi, K.; Westover, T. L.; Fisher, T. S.; Weiner, B. R.; Morell, G., Thermionic emission energy distribution from nanocrystalline diamond films for direct thermal-electrical energy conversion applications, J. Appl. Phys. 106 (2009) 043716.

[61] Koeck, F. A. M.; Nemanich, R. J.; Lazea, A.; Haenen, K., Thermionic electron emission from low work-function phosphorus doped diamond films, Diamond and Related Materials 18 (5 - 8) (2009) 789-791.

[62] F.A.M. Koeck, R.J. Nemanich Diamond \& Related Materials 14 (2005) 2051 2054

F.A.M. Köck, J.M. Garguilo, Billyde Brown, R.J. Nemanich Diamond and Related Materials 11 (2002) 774-779

F.A.M. Koeck, J.M. Garguilo, R.J. Nemanich Diamond \& Related Materials 13 (2004) 2052- 2055

Franz A.M. Koeck, Robert J. Nemanich

Diamond \& Related Materials 15 (2006) 217 - 220

Franz A.M. Koeck, Robert J. Nemanich, Andrada Lazea, Ken Haenen

Diamond \& Related Materials 18 (2009) 789-791 
[63] Nemanich, R. J.; Bergman, L.; Turner, K. F.; Vanderweide, J.; Humphreys, T. P., Properties of interfaces of diamond, Physica B 185 (1-4) (1993) 528-538.

[64] Benndorf, C.; Hadenfeldt, S.; Luithardt, W.; Zhukov, A., Photoelectron spectroscopic investigations and exoelectron emission of CVD diamond surfaces modified with oxygen and potassium, Diamond and Related Materials, 5 (6-8) (1996) 784-789.

[65] Vanderweide, J.; Nemanich, R. J., Influence of interfacial hydrogen and oxygen on the Schottky barrier height of nickel on (111) and (100) diamond surfaces, Phys. Rev. B 49 (19) (1994) 13629-13637.

[66] Geis, M. W.; Twichell, J. C.; Macaulay, J.; Okano, K., Electron field emission from diamond and other carbon materials after $\mathrm{H} 2, \mathrm{O} 2$, and Cs treatment, Appl. Phys. Lett. 67(9) (1995) 1328-1330.

[67] Wong, K. W.; Wang, Y. M.; Lee, S. T.; Kwok, R. W. M., Negative electron affinity on polycrystalline diamond surface induced by lithium fluoride deposition, Diamond and Related Materials 8 (10) (1999) 1885-1890.

[68] Wong, K. W.; Wang, Y. M.; Lee, S. T.; Kwok, R. W. M., Lowering of work function induced by deposition of ultra-thin rubidium fluoride layer on polycrystalline diamond surface, Appl. Surf. Sci. 140 (1-2) (1999) 144-149.

[69] Dumpala, S.; Jasinski, J. B.; Sumanasekera, G. U.; Sunkara, M. K., Synthesis of Conical Carbon Nanotube Arrays: Mechanistic Aspects and Growth on Foil Substrates, Carbon 49 (2011) $2725-2734$.

Santoshrupa Dumpala, Abdelilah Safir, David Mudd, Robert W. Cohn, Mahendra K. Sunkara, Gamini U. Sumanasekera, Diamond Relat. Mater. 18 (2009) 
$1262-1266$.

[70] Sherehiy, A.; Dumpala, S.; Safir, A.; Mudd, D.; Arnold, I.; Cohn, R. W.; Sunkara, M. K. Sumanasekera, G. U., Thermionic emission properties and the work function determination of arrays of conical carbon nanotubes, Diamond and Related Materials 34 (2013) 1-8.

[71] Chernomordik, B.; Dumpala, S.; Chen, Z.Q.; Sunkara, M.K., Nanodiamond Tipped and Coated Conical Carbon Tubular Structures, Chem. Vap. Depos. 14 (2008) 256-262.

[72] Rentenberger, S.; Vollmer, A.; Zojer, E.; Schennach, R.; Koch, N., UV/ozone treated $\mathrm{Au}$ for air-stable, low hole injection barrier electrodes in organic electronics, J. Appl. Phys. 100 (5) (2006) 053701.

[73] Reimann, A. L. Thermionic Emission; John Wiley and Sons, Inc.: New York, 1934; Chapter 1, p 61.

[74] Modinos, A. Field, Thermionic, and Secondary Electron Emission Spectroscopy; Plenum Press: New York, 1984; Chapter 1, pp 20-22.

[75] Koeck, F. A. M.; Nemanich, R. J., Emission characterization from nitrogen-doped diamond with respect to energy conversion, Diamond \& Related Materials (15) (2006) $217-220$.

[76] Liu, P.; Sun, Q.; Zhu, F.; Liu, K.; Jiang, K.; Liu, L.; Li, Q.; Fan, S., Measuring the Work Function of Carbon Nanotubes with Thermionic Method, Nano Leterrs 8 (2) (2008) 647-651.

[77] Köck, F. A. M.; Garguilo, J. M.; Brown, B. and Nemanich, R. J., Enhanced lowtemperature thermionic field emission from surface-treated $\mathrm{N}$-doped diamond films, Diamond and Related. Materials. 11 (2002) 774. 
[78] Koeck, F. A. M.; Garguilo, J. M. and Nemanich, R. J., On the thermionic emission from nitrogen-doped diamond films with respect to energy conversion, Diamond and Related Materials 13 (2004) 2052.

[79] Ristein, J.; Maier, F.; Riedel, M.; Cui, J.B. and Ley, L., Surface Electronic Properties of Diamond, Phys. Stat. Sol. (a) 181 (2000) 65.

[80] Diederich, L.; Kuttel, O. M.; Ruffieux, P.; Pillo, T.; Aebi, P.; Schlapbach, L., Photoelectron emission from nitrogen- and boron-doped diamond (100) surfaces, Surf. Sci. 417 (1998) 41-52.

[81] S. Vaddiraju, H. Chandrasekaran, M. K. Sunkara, J. Am. Chem. Soc., 125 (36), $10792(2003)$

[82] J. Thangala, S. Vaddiraju, R. Bogale, R. Thurman, T. Powers, B. Deb, and M.K. Sunkara, ECS Transactions, 3 (9) 97-105 (2006)

[83] Kim H, Senthil K, Yong K - Nanoscale Res Lett (2009

[84] A. Baserga et al., Thin Solid Films 515(16), 6465-6469 (2007)

[85] J. Díaz-Reyes et al., Superficies y Vacío 21(2), 12-17, (2008) 


\section{APPENDIX A}

Reprinted from Diamond and Related Materials, 34, Sherehiy, A.; Dumpala, S.; Safir, A.; Mudd, D.; Arnold, I.; Cohn, R. W.; Sunkara, M.; Sumanasekera, G. U.,

" Thermionic emission properties and the work function determination of arrays of

conical carbon nanotubes", 1 - 8, Copyright (April 2013), with permission from Elsevier. 


\section{APPENDIX B}

Reprinted from Diamond and Related Materials, 50, Sherehiy, A.; Dumpala, S.; Sunkara,

M. K.; Cohn, R. W.; Sumanasekera, G. U., "Thermionic emission from phosphorus (P)

doped diamond nanocrystals supported by conical carbon nanotubes and ultraviolet

photoelectron spectroscopy study of P-doped diamond films“, 66 - 76, Copyright

(November 2014), with permission from Elsevier. 


\title{
CURICULUM VITAE
}

\author{
Andriy Sherehiy \\ 1424 Rufer Ave, Apt 2 \\ Louisville, KY 40204
}

E-mail: asherehiy@gmail.com a0sher02@louisville.edu

\section{EDUCATION}

\section{University of Louisville, Louisville, KY, USA}

- PhD, 12/2014

Thesis: Thermionic emission properties of the novel carbon nanostructures.

Advisor: Dr Gamini Sumanasekera, Co-Advisor: Dr Robert W Cohn GPA: 3.597

- M.S., Physics, 08/2010

Thesis: Thermopower measurements of large area CVD graphene. Advisor: Dr Gamini Sumanasekera GPA: 3.597

University of Rzeszow, Rzeszow, Poland

- M.S., Physics, 06/2008

- B.S., Physics, 06/2006

\section{RESEARCH EXPERIENCE}

\section{Department of Physics and Astronomy, University of Louisville and} Conn Center for Renewable Energy Research, University of Louisville PhD Research, 2010 - 2014

Project: Novel carbon and diamond nano structures for thermionic conversion.

- Studied thermionic emission properties and determined work function of the nanostructured carbon materials:

- Work function reduction due to field penetration effect in case of Conical Carbon Nanotubes (CCNT) array grown on platinum wire and graphite foil Article has been published in "Diamond and Related Materials".

- Reduced value of work function of CCNTs coated with phosphorous doped diamond nanocrystals and phosphorous doped diamond films. Article has been published in "Diamond and Related Materials". 
- Synthesized materials for field and thermionic emission measurements: array of tungsten oxide nanowires by thermal Chemical Vapor Deposition (CVD); undoped and phosphorous doped diamond films and individual crystals by Microwave Plasma Assisted CVD

- Trained and supervised undergraduate and graduate students

\section{Department of Physics and Astronomy, University of Louisville, Master Degree Research, 2009 - 2010}

Project: Thermoelectric power and resistivity studies of the graphene grown by Chemical Vapor Deposition (CVD).

- Studied charge transfer effect of large area graphene by in situ monitoring of the thermoelectric power and 4 probe resistance.

Department of Physics, Rzeszow University, 2005 - 2008

Undergraduate and Master Degree Research

Project: Theoretical studies of the Quantum Entanglement in simple Heisenberg model. (Measures of Quantum Entanglement).

- Analysis of quantum entanglement in 3 body Heisenberg model by application of two separate methods of determining quantum entanglement measures.

\section{TEACHING EXPERIENCE}

Department of Physics and Astronomy, University of Louisville 2008 - 2010 Teaching Assistantship

- Conducted laboratories for the undergraduate students

\section{LABORATORY AND INSTRUMENTATION SKILLS}

- Labview and data acquisition systems

- High vacuum systems $\left(10^{-6}-10^{-8}\right.$ torr $)$

- Field emission measurements

- Thermionic Emission measurements

- Electron transport measurements in low and high temperatures $(10 \mathrm{~K}-600 \mathrm{~K})$ :

○ 4-probe resistance, Hall measurement, Thermopower, thermal conductivity, magnetoresistance;

- Chemical Vapor Deposition systems

- Microwave Plasma Chemical Vapor Deposition systems - SEKI Technotron AX5010 and SEKI Technotron AX5200-S ECR

- RF Plasma Chemical Vapor Deposition system

- Atomic Layer Deposition system - Cambridge NanoTech Savannah 100

- Scanning Electron Microscopy: Zeiss Supra and FEI NOVA Nano SEM 600

- Raman characterization - Renishaw inVia Raman Microscope 
- Atomic Force Microscope - XE-100 Park Systems

- Ultraviolet Photoelectron Spectroscopy and X ray Photoelectron Spectroscopy measurements - ultra high vacuum surface science facility (VG Scientific/RHK Technology)

\section{SOFTWARE SKILLS}

Igor, Origin, Maple.

\section{PUBLICATIONS:}

Dustin R. Cummins, Rajesh Kappera, Andriy Sherehiy, Alejandro Martinez, Jacek Jasinski, Ulises Martinez, Manish Chhowalla' Gamini Sumanasekara, Aditya Mohite, Gautam Gupta, and Mahendra K. Sunkara, Electrochemical Effects of Hydrazine on $\mathrm{MoS}_{2}$ Nanowires, manuscript in preparation, (2014)

I. M. Dharmadasa, O. K. Echendu, F. Fauzi, H. I. Salim, N. A. Abdul-Manaf, J. B. Jasinski, A. Sherehiy and G. U. Sumanasekera, Study of Fermi level movement using Ultra-violet Photoelectron Spectroscopy during $\mathrm{CdCl}_{2}$ treatment of CdTe thin films, Under review, Materials Chemistry and Physics (2014)

Menaka Jha; Ruvini Dharmadasa; Gabriel Draper; Andriy Sherehiy; Gamini U. Sumanasekera; Delaina Amos; Thad Druffel, Solution Phase Synthesis and Intense Pulsed Light Sintering of a Copper Conductive Ink with an Encapsulating Nickel Barrier, Under review, Chemistry of Materials (c) (2014)

Andriy Sherehiy, Santoshrupa Dumpala, Mahendra K. Sunkara, Jacek B. Jasinski, Robert W. Cohn, Gamini U. Sumanasekera, Thermionic emission from phosphorus $(P)$ doped diamond nanocrystals supported by conical carbon nanotubes and Ultraviolet Photoelectron Spectroscopy study of P-doped diamond films, In Press, Diamond and Related Materials (2014), DOI: 10.1016/j.diamond.2014.09.005

Ruwantha Jayasinghe, Arjun Kumar Thapa, Ruchira R. Dharmasena, Tu Quang Nguyen, Bhabendra K. Pradhan, Hem Sharma Paudel, Jacek B. Jasinski, Andriy Sherehiy, Masaki Yoshio, G.U. Sumanasekera, Optimization of Multi-Walled Carbon Nanotube based CFx electrodesfor improved primary and secondary battery performances, Journal of Power Sources 253, 404-411 (2014)

Ruwantha Jayasingha, Andriy Sherehiy, Shi-Yu Wu, and G. U. Sumanasekera, In Situ Study of Hydrogenation of Graphene and New Phases of Localization between Metal-Insulator Transitions, Nano Letters, 13, 5098-5105 (2013)

Andriy_Sherehiy, Santoshrupa Dumpala, Abdelilah Safir, David Mudd, Ivan Arnold, Robert W. Cohn, Mahendra K. Sunkara ${ }_{2}$ Gamini U. Sumanasekera ${ }_{2}$ Thermionic emission properties and the work function determination of arrays of conical carbon nanotubes, 
Diamond and Related Materials, 34, 1-8 (2013)

Vidhya Chakrapani, Gamini U. Sumanasekera, Buddhika Abeyweera, Andriy Sherehiy, and John C. Angus, Electrochemically Induced p-Type Conductivity in Carbon Nanotubes, ECS Solid State Letters, 2 (11) M1-M4 (2013)

Anton N Sidorov, Kurt Gaskill, Marco Buongiorno Nardelli, Joseph L Tedesco, Rachel L Myers-Ward, Charles R Eddy, Thushari Jayasekera, Harry K W Kim, Ruwantha Jayasingha, Andriy Sherehiy, Robert Stallard, Gamini U Sumanasekera, Charge transfer equilibria in ambient-exposed epitaxial graphene on (0001) $6 \mathrm{H}$-SiC, Journal of Applied Physics, 111, 113706 (2012)

Anton N. Sidorov, Andriy Sherehiy, Ruwantha Jayasinghe, Robert Stallard, Daniel K. Benjamin, Qingkai Yu, Zhihong Liu, Wei Wu, Helin Cao, Yong P. Chen, Zhigang Jiang, Gamini U. Sumanasekera, Thermoelectric power of graphene as surface charge doping indicator, Applied Physics Letters, 99(1), 013115-013115-3 (2011)

\section{SELECTED PRESENTATIONS:}

2010 "Thermoelectric properties of CVD grown large area graphene", American Physical

Society March Meeting, Portland, Oregon

2011 "Conical carbon nanotubes area synthesis, electrochemical and field emission characteristics", Poster Session, Conn Center for Renewable Energies Research Workshop, Louisville, KY

2011 "Thermionic emission properties of surface modified conical carbon nanotubes" American Physical Society March Meeting, Dallas, Texas

2012 "Thermionic emission properties and the work function determination of surface modified conical carbon nanotubes", The First International Conference on Materials, Energy and Environments, University of Toledo, Toledo, Ohio

2013 "Thermionic emission properties and the work function determination of phosphorous doped diamond crystals supported by conical carbon nanotubes" Symposium on Nanostructured Materials, University of Rzeszow, Rzeszow, Poland

2014 "Thermionic emission properties of the novel carbon nanostructures", Workshop on Thermionic Energy Conversion for Space and Earth, NASA Johnson Space Center, Houston, Texas 


\section{OTHER EXPERIENCE}

2009 - 2013, Department of Physics and Astronomy representative at Graduate Student Council, University of Louisville

2009 Referee at Louisville Regional Science Fair (Physical Science) 